\title{
Double Hopf Bifurcation with Huygens Symmetry*
}

\author{
P. M. Kitanov ${ }^{\dagger}$, W. F. Langford ${ }^{\dagger}$, and A. R. Willms ${ }^{\dagger}$
}

\begin{abstract}
Double Hopf bifurcations have been studied prior to this work in the generic nonresonant case and in certain strongly resonant cases, including 1:1 resonance. In this paper, the case of symmetrically coupled identical oscillators, motivated by the classic problem of synchronization of Huygens' clocks, is studied using the codimension-three Elphick-Huygens equivariant normal form presented here. The focus is on the effect that the Huygens symmetry assumption has on the dynamic behavior of the system. Periodic solutions include the classical in-phase and anti-phase normal modes that are forced by the symmetry, as well as pairs of mixed mode phase-locked periodic solutions. The escapement paradox is explained. A theorem based on topological degree theory establishes the existence of quasi-periodic solutions in an invariant 3-torus that resembles a 2-torus slightly thickened to a solid toroidal shell, with the two principal radii of the 2-torus slowly modulated in time - that is, a toroidal breather. Secondary bifurcations from the in-phase and anti-phase normal modes are explored, of codimension one and two, and it is shown that an Arnold tongue plays a fundamental role in the determination of whether secondary bifurcation gives birth to phase-locked periodic solutions or to quasi-periodic solutions. Detailed numerical analysis, using MATLAB, extends the local bifurcation analysis to a more global picture that includes coexistence of multiple stable solutions and a "swallowtail" bifurcation of periodic solutions.
\end{abstract}

Key words. double Hopf bifurcation, symmetry, equivariant bifurcation theory, nonlinear resonance, coupled identical oscillators, Huygens' clocks, toroidal breather

AMS subject classifications. 34C23, 34D06, 37G15, 37G40, 70K30

DOI. $10.1137 / 110839461$

1. Introduction. The synchronization of coupled oscillators was first studied by Huygens in the 17th century [20]. Huygens was a manufacturer of very accurate pendulum clocks. He observed that if two of his clocks were weakly coupled, for example by placing them next to each other on a wall, after a short time they synchronized. Specifically, he wrote that after about 30 minutes, his two pendula swung with exactly the same period of oscillation, but with opposite displacements and velocities. Today, this type of coupled oscillation is called anti-phase synchronization. Huygens was not able to explain his observation, and it has been a topic of investigation to this day; see [3, 4, 5, 19, 23, 27, 30, 31, 32] and other references therein. Following Huygens, it has been found that other types of synchronization of identical oscillators are possible, for example in-phase synchronization, in which the two oscillators have the same period as well as the same phase [5, 19, 27, 30, 31].

In this paper it is assumed that the two oscillators are identical and that their coupling is symmetric; that is, the system is unchanged if the two oscillators are interchanged. This

\footnotetext{
${ }^{*}$ Received by the editors July 5, 2011; accepted for publication (in revised form) by E. Knobloch September 26, 2012; published electronically January 24, 2013. This research was supported in part by the Natural Sciences and Engineering Research Council of Canada.

http://www.siam.org/journals/siads/12-1/83946.html

${ }^{\dagger}$ Department of Mathematics and Statistics, University of Guelph, Guelph, ON, Canada N1G 2W1 (pkitanov@uoguelph.ca, wlangfor@uoguelph.ca, awillms@uoguelph.ca).
} 
symmetry assumption is motivated by Huygens' clocks. It is assumed also that the two oscillators are near the onset of periodic oscillations, via Hopf bifurcations. Then the fundamental role played by the symmetry in determining the dynamic behaviors and bifurcations exhibited by such coupled oscillators is explored, using the method of equivariant normal forms. Even though this symmetry is very elementary, it profoundly changes the typical behavior of the coupled oscillator system. This paper is based on the results of [22].

The literature on systems of symmetrically coupled oscillators is large. For example, Swift [34] studied Hopf bifurcation of four identical oscillators with $D_{4}$ symmetry; see also [36, 29]. In that case, the bifurcation has codimension one, not three, and the cubic normal form has half as many independent coefficients, so the range of dynamics is not as rich as in the present case. For another example, phase models for symmetrically coupled oscillators, where the coupling is weak, have been studied in $[2,6]$. Phase models require that the amplitudes of the oscillators remain of order one, so they rule out the possibility of Hopf bifurcation, which is a focus of this paper.

Definition 1.1. The dynamical system defined by $\dot{x}=f(x)$ on the state space $\mathbb{R}^{m}$ is equivariant with respect to the group $\Gamma$ acting on $\mathbb{R}^{m}$ if

$$
f(\gamma x)=\gamma f(x) \quad \forall \gamma \in \Gamma, x \in \mathbb{R}^{m} .
$$

In the case of Huygens' system, $m=2 n$, and the individual oscillators (clocks) have identical state spaces of dimension $n \geq 2$, written here as

$$
v_{1} \in V_{1}, \quad v_{2} \in V_{2}, \quad V_{1}=V_{2}=\mathbb{R}^{n} .
$$

Then the state vector of the coupled system is $x=\left[\begin{array}{l}v_{1} \\ v_{2}\end{array}\right]$ in the state space $V_{1} \times V_{2}=\mathbb{R}^{2 n}$, and the operator corresponding to interchange of the two clocks is represented by the $2 n \times 2 n$ permutation matrix

$$
\sigma=\left[\begin{array}{cc}
0 & I_{n} \\
I_{n} & 0
\end{array}\right]
$$

where $I_{n}$ is the $n \times n$ identity matrix. This permutation generates the two-element group

$$
\Gamma_{H}=\left\{I_{2 n}, \sigma\right\} .
$$

Definition 1.2. A dynamical system on $\mathbb{R}^{2 n}$ that is equivariant with respect to the group $\Gamma_{H}$ is said to have Huygens symmetry.

Throughout this article it is assumed that the system has Huygens symmetry.

Independent of this Huygens symmetry, a classical pendulum has an internal reflection symmetry in which both displacement and velocity are symmetric on reflection through zero; that is, $f$ is an odd function: $f(-x)=-f(x)$. The symmetry operator is represented by the matrix $-I_{2 n}$. The four-element group generated by these two symmetry operators is called the odd-Huygens symmetry group and is denoted

$$
\Gamma_{ \pm H}=\left\{I_{2 n}, \sigma,-I_{2 n},-\sigma\right\} .
$$


Remark 1.3. In the literature there has been some disagreement about the importance of the reflection symmetry in models of Huygens' clocks, because most clocks (including Huygens') are driven by an escapement mechanism that pushes the pendulum in only one direction, thus breaking the reflection symmetry. For example, the models of Bennett et al. [4] and Senator [32] specifically include an escapement mechanism that breaks the reflection symmetry and thus have only the Huygens symmetry (4), while the models of Belykh and Pankratova [3], Blekhman [5], Korteweg [23], and Pantaleone [30] all have the odd-Huygens symmetry group (5). Although the symmetry groups (4) and (5) seem very different, it has been found that the models with a symmetry-breaking escapement mechanism have dynamic behavior that is qualitatively similar to those that possess the extra reflection symmetry in (5). We explore this issue further in sections 2.2 and 3.4, where it is called the escapement paradox.

The second major assumption in this paper is that the system is near a point of double Hopf bifurcation with 1:1 resonance. In the case of pendulum clocks that are designed to oscillate with very low damping (friction), it is reasonable to assume that the linearization will have complex eigenvalues with very small real part, which is a requirement for Hopf bifurcation. Since the clocks are identical, one expects Hopf bifurcation to be double and near 1:1 resonance. We show that with the assumed Huygens symmetry this double bifurcation has codimension three. The analysis is carried out in general, not just for pendulum clocks, so that the results are applicable to any system of two coupled oscillators near double Hopf bifurcation with the assumed Huygens symmetry, for example, other mechanical oscillators [5, 27, 31], electronic circuits [31], or models of coupled identical neural oscillators [7, 11, 18].

Two related cases of double Hopf bifurcation have been studied in detail: the nonresonant double Hopf bifurcation [16, 21, 25] and the generic 1:1 resonant double Hopf bifurcation (with no imposed symmetry) $[15,35,37]$. The additional assumption here of Huygens symmetry forces the Jacobian matrix to be semisimple, even at 1:1 resonance where the eigenvalues are double, unlike the case of generic 1:1 resonance where the Jacobian is nonsemisimple. We show that, in a certain limiting case, the typical behavior exhibited by the classical nonresonant double Hopf bifurcation is reproduced in this symmetric case, while in fact there is little in common between the two cases of 1:1 resonance with and without Huygens symmetry. In addition, the present case exhibits unique phenomena not seen before.

The outline of this paper is as follows. In section 2, it is shown that the assumed Huygens symmetry gives rise to both in-phase and anti-phase periodic solutions, as well as that the Jacobian is semisimple; these are model-independent results. In section 3, the codimensionthree Elphick-Huygens normal form is derived, for double Hopf bifurcation with Huygens symmetry, together with its unfolding. In section 4, in addition to the well-known in-phase and anti-phase normal mode solutions, mixed mode phase-locked periodic solutions are found, in symmetric pairs. In section 5 , it is proven that quasi-periodic solutions on invariant tori exist in the form of a "toroidal breather." See the accompanying video of this breather (83946_01.avi [local/web 164MB]). In section 6, secondary bifurcations from the in-phase and anti-phase normal mode solutions are explored. These include codimension-one pitchfork and saddlenode bifurcations as well as codimension-two bifurcations of Bogdanov-Takens, pitchforksaddle node, and Bautin type. In section 6.3, detailed numerical analysis using MATLAB extends the local bifurcation analysis to a more global picture. Coexistence of different stable solutions is demonstrated, away from the local bifurcations. Numerical evidence suggests 
that the transition from two to four phase-locked periodic solutions occurs via a swallowtail bifurcation.

2. Model-independent properties. Symmetries can force the existence of properties of the trajectories of a dynamical system that are independent of the details of the physical laws that otherwise govern the model $[8,13,14]$. In fact, it is not necessary to solve (or even write down) the differential equations in order to know that these properties hold. Such properties therefore are called model-independent.

Consider the initial value problem

$$
\dot{x}=f(x), \quad x(0)=x_{0},
$$

where $x \in \mathbb{R}^{m}, f: \mathbb{R}^{m} \rightarrow \mathbb{R}^{m}$, and $f$ is a smooth function (infinitely differentiable). Then for each $x_{0} \in \mathbb{R}^{m}$ the initial value problem (6) has a unique solution $\phi\left(t, x_{0}\right)$ on some $t$ interval $I \subseteq \mathbb{R}$. (Here $I$ may depend on $x_{0}$.) The set of mappings $\phi_{t}$ on $\mathbb{R}^{m}$ defined by $\phi_{t}\left(x_{0}\right)=\phi\left(t, x_{0}\right)$ is called the flow of the differential equation (6). If $\phi$ is a given solution of (6), define the orbit (or flow-orbit, to distinguish it from the group-orbit below) of $\phi$ to be the set $\{\phi(t)\} \subset \mathbb{R}^{m}$ for all $t$ such that $\phi(t)$ is defined (usually all $t \in \mathbb{R}$ ).

It is assumed throughout that $f$ is equivariant with respect to a group $\Gamma \subseteq \operatorname{GL}\left(\mathbb{R}^{m}\right)$, as in (1). For any $x \in \mathbb{R}^{m}$, define the group-orbit of $x$ to be $\{\gamma x \mid \gamma \in \Gamma\}$. The following result is standard $[13,14]$.

Lemma 2.1. For all $\gamma \in \Gamma$ the function $x=\phi(t)$ is a solution of the differential equation in (6) if and only if $\gamma \phi(t)$ is a solution. The flow-orbits of $\phi$ and $\gamma \phi$ are either identical sets or disjoint sets. The flow $\phi_{t}$ is $\Gamma$-equivariant, that is

$$
\gamma \phi_{t}(x)=\phi_{t}(\gamma x)
$$

Proof. Since the group action does not depend on time, it follows that $\gamma$ and $\frac{d}{d t}$ commute; i.e., $\gamma \frac{d \phi}{d t}=\frac{d \gamma \phi}{d t}$. Suppose that $\phi(t)$ is a solution of the differential equation in (6), i.e., $\frac{d}{d t} \phi(t)=$ $f(\phi(t)), t \in \mathbb{R}$. If we apply $\gamma$ on both sides of this, we get

$$
\gamma \frac{d \phi(t)}{d t}=\gamma f(\phi(t)) \quad \text { iff } \quad \frac{d}{d t}(\gamma \phi(t))=f(\gamma \phi(t)) .
$$

Therefore $\phi$ is a solution of the differential equation in (6) if and only if $\gamma \phi$ is a solution of the differential equation in (6). If the orbits of $\phi$ and $\gamma \phi$ have one point in common, choose this point to be $x_{0}$ in (6), and then the orbits are identical by the uniqueness theorem for (6). Next we show that $\phi_{t}$ is equivariant for each $t \in \mathbb{R}$. Consider the two solutions $\phi_{t}(\gamma x)$ and $\gamma \phi_{t}(x)$. At $t=0$,

$$
\left.\gamma \phi_{t}(x)\right|_{t=0}=\gamma \phi_{0}(x)=\gamma x \quad \text { and }\left.\quad \phi_{t}(\gamma x)\right|_{t=0}=\phi_{0}(\gamma x)=\gamma x .
$$

This shows that $\gamma \phi_{t}(x)$ and $\phi_{t}(\gamma x)$ satisfy the same initial value problem, and from the uniqueness theorem it follows that $\gamma \phi_{t}(x)=\phi_{t}(\gamma x)$ for all $t$. 
2.1. Isotypic decomposition. We begin with some basic definitions.

Definition 2.2. A subspace $V \subseteq \mathbb{R}^{m}$ is $\Gamma$-invariant if $\gamma V \subseteq V$ for all $\gamma \in \Gamma$.

Definition 2.3. A subspace $V \subseteq \mathbb{R}^{m}$ is $\Gamma$-irreducible if $V$ is $\Gamma$-invariant and the only subspaces of $V$ that are $\Gamma$-invariant are $\{0\}$ and $V$ itself.

Definition 2.4. Let $\Gamma \in \mathrm{GL}\left(\mathbb{R}^{m}\right)$, and let $V_{j}, V_{k}$ be subspaces of $\mathbb{R}^{m}$. Then $V_{j}$ and $V_{k}$ are called $\Gamma$-isomorphic if there exists a linear isomorphism $A: V_{j} \longleftrightarrow V_{k}$ such that $A(\gamma x)=$ $\gamma A(x)$ for all $x \in V_{j}, \gamma \in \Gamma$.

Definition 2.5. Let $\Gamma \in \mathrm{GL}\left(\mathbb{R}^{m}\right)$, and let $V_{j}$ be a $\Gamma$-irreducible subspace in $\mathbb{R}^{m}$. Let $W_{j}$ be the sum of all $\Gamma$-irreducible subspaces that are $\Gamma$-isomorphic to $V_{j}$. Then $W_{j}$ is called an isotypic component of $\mathbb{R}^{m}$ for $\Gamma$ of type $V_{j}$.

The following is a general result in group representation theory $[13,14]$.

Theorem 2.6. The vector space $\mathbb{R}^{m}$ can be written uniquely as a direct sum of the isotypic components $W_{j}$ of $\mathbb{R}^{m}$ for $\Gamma$; that is,

$$
\mathbb{R}^{m}=W_{1} \oplus \cdots \oplus W_{s} .
$$

This is called the isotypic decomposition of $\mathbb{R}^{m}$ for $\Gamma$.

Define two subspaces of $\mathrm{R}^{2 n}$ by

$$
W_{+}=\left\{\left[\begin{array}{l}
v \\
v
\end{array}\right] \mid v \in \mathbb{R}^{n}\right\}, \quad W_{-}=\left\{\left[\begin{array}{r}
v \\
-v
\end{array}\right] \mid v \in \mathbb{R}^{n}\right\} .
$$

Then

$$
\mathbb{R}^{2 n}=W_{+} \oplus W_{-}
$$

is the isotypic decomposition of $\mathrm{R}^{2 n}$ for $\Gamma_{H}$ in (4).

A special type of invariant subspace is presented by the fixed-point subspaces.

Definition 2.7. Let $\Sigma$ be a subgroup of $\Gamma$. Then the fixed-point subspace of $\Sigma$ is defined as

$$
\operatorname{Fix}(\Sigma)=\left\{x \in \mathbb{R}^{m} \mid \gamma x=x \forall \gamma \in \Sigma\right\} .
$$

That is, $\operatorname{Fix}(\Sigma)$ is the set of all states $x$ which have the symmetries of $\Sigma$.

Since a group action is a linear operation, it follows that $\operatorname{Fix}(\Sigma)$ is a linear subspace. An important property of fixed-point subspaces is their flow-invariance.

Definition 2.8. An open set $S \subseteq \mathbb{R}^{m}$ is invariant with respect to the flow $\phi_{t}$ (or is flowinvariant) if the flow satisfies

$$
\phi_{t}(S) \subseteq S
$$

for all $t$ such that the flow is defined.

For the initial value problem (6) this means that if $x_{0} \in S$, then the entire orbit of $x(t)$ remains in $S$. Note that isotypic components as in (7) and (9) are not in general flow-invariant. The following theorem is well known $[13,14]$.

Theorem 2.9. $\operatorname{Fix}(\Sigma)$ is flow-invariant for any subgroup $\Sigma \subseteq \Gamma$.

Proof. Consider the initial value problem (6), where $f$ is equivariant with respect to the group $\Gamma$. For $x \in \operatorname{Fix}(\Sigma)$, by the $\Gamma$-equivariance property in Lemma 2.1, we have

$$
\gamma \phi_{t}(x)=\phi_{t}(\gamma x)=\phi_{t}(x) \quad \forall t .
$$

Therefore $\phi_{t}(x) \in \operatorname{Fix}(\Sigma)$ for all $t$, which means that $\operatorname{Fix}(\Sigma)$ is flow-invariant. 
Now consider the Huygens group $\Gamma=\Gamma_{H}$. From Theorem 2.9, $\operatorname{Fix}\left(\Gamma_{H}\right)=W_{+}$is a flow-invariant subspace of solutions of (6) with permutation symmetry $v_{1}(t)=v_{2}(t)$ for all $t$, which implies that the two oscillators are always in-phase in $W_{+}$. However, the groupinvariant subspace $W_{-}$, which is an isotypic component but not a fixed-point subspace, is not flow-invariant in general (as is easily verified by counterexample).

Next consider systems as studied in $[3,5,23,30]$ with the odd-Huygens symmetry group $\Gamma_{ \pm H}(5)$, which has the two fixed-point subspaces

$$
\operatorname{Fix}\left(\Sigma_{\sigma}\right)=\left\{\left[\begin{array}{c}
v \\
v
\end{array}\right] \mid v \in \mathbb{R}^{n}\right\} \quad \text { and } \operatorname{Fix}\left(\Sigma_{-\sigma}\right)=\left\{\left[\begin{array}{c}
v \\
-v
\end{array}\right] \mid v \in \mathbb{R}^{n}\right\} .
$$

Then the isotypic decomposition of $\mathbb{R}^{2 n}$ for $\Gamma_{ \pm H}$ given by (5) is

$$
\mathbb{R}^{2 n}=\operatorname{Fix}\left(\Sigma_{\sigma}\right) \oplus \operatorname{Fix}\left(\Sigma_{-\sigma}\right),
$$

where $\operatorname{Fix}\left(\Sigma_{\sigma}\right) \equiv W_{+}, \operatorname{Fix}\left(\Sigma_{-\sigma}\right) \equiv W_{-}$, and now both of these isotypic components are flowinvariant. Solutions in $W_{+}$have in-phase symmetry, as was the case for $\Gamma_{H}$, but now in addition all solutions in $W_{-}$satisfy $\sigma x(t)=-x(t)$ for all $t$, which we call antipermutation symmetry, to distinguish it from the anti-phase symmetry in Definition 2.10 below. So, if (6) is $\Gamma_{ \pm H}$-equivariant, then any solution of the system can be written uniquely as a sum of in-phase and antipermutation components. If an initial point $x_{0}$ is in one of these fixed-point subspaces at $t=0$, then the solution of (6) remains in that subspace and preserves its symmetry for all $t$. Thus the isotypic decomposition (11) into fixed-point subspaces explains the existence of both in-phase and antipermutation solutions for systems that have the symmetry group $\Gamma_{ \pm H}$ as in $[3,5,23,30]$.

2.2. The escapement paradox. Because of the escapement mechanism, Huygens' clocks possessed only the symmetry group $\Gamma_{H}$, not the larger group $\Gamma_{ \pm H}$. For $\Gamma_{H}$, the isotypic decomposition (9) guarantees the existence of in-phase oscillations in $W_{+}$, which, however, were not observed by Huygens. Paradoxically, $\Gamma_{H}$ does not guarantee the existence of solutions with antipermutation symmetry, which it seems were observed and reported by Huygens. This is the escapement paradox. In order to explain the escapement paradox, one must recall that the oscillations observed by Huygens were periodic. Huygens symmetry alone does not force solutions to be periodic. Therefore, for the purposes of this section, let us hypothesize that periodic solutions exist, with minimum period $T>0$. (It will be proven in section 4 that such periodic solutions do exist, thus rendering this hypothesis redundant.)

Define a periodic orbit to be the closed path (set) in the state space traced by a periodic solution $x(t)$ as $t$ varies from 0 to $T$. Any periodic orbit of an autonomous ODE has a phase-shift symmetry, defined as follows. Let $x(t)$ be a $T$-periodic solution of (6), that is, $x(t)=x(t+T)$ for all $t$, and there is no smaller $T>0$ for which this is true. For any $\psi \in[0,2 \pi]$, define the corresponding phase-shift operator $\psi$ by

$$
\psi x(t)=x\left(t+\psi \frac{T}{2 \pi}\right) .
$$

Then, $\psi x$ is $T$-periodic whenever $x$ is, and furthermore the solutions $\psi x(t)$ and $x(t)$ define the same periodic orbit. Denote the circle group $S^{1}=\{\psi \in \mathbb{R}(\bmod 2 \pi)\}$. Then $(12)$ is a 
representation of $S^{1}$ acting on the space of $T$-periodic functions $x(t)$ with values in $\mathbb{R}^{n}$. Any $T$-periodic orbit is invariant under this $S^{1}$ action and is said to have $S^{1}$ phase-shift symmetry (or temporal symmetry).

Now assume that (6) has the Huygens permutation symmetry $\sigma \in \Gamma_{H}$ from (3) but not the reflection symmetry $-I$, as in $[4,20,32]$. To contrast with the temporal symmetry $S^{1}$, call $\Gamma_{H}$ in (4) a spatial symmetry group. If $O_{x}$ is a periodic orbit with period $T$, corresponding to the solution $x=\phi(t)$, then by Lemma 2.1, $\sigma O_{x}$ is also a periodic orbit with period $T$, and these two orbits are either disjoint sets or the same set. Both of the groups $\Gamma_{H}$ and $S^{1}$ act on orbits of (6) and they commute, so we may consider the combined group of spatio-temporal symmetries defined by

$$
\Gamma_{S T}=S^{1} \times \Gamma_{H}=\left\{\psi \gamma \equiv(\psi, \gamma) \mid \psi \in S^{1}, \gamma \in \Gamma_{H}\right\}, \text { where } \psi \gamma x(t)=\gamma x\left(t+\psi \frac{T}{2 \pi}\right) .
$$

The importance of spatio-temporal symmetries in bifurcation problems was recognized by Golubitsky and Stewart [13, 14]. Of particular interest here is the subgroup of $S^{1}$ of order 2 that corresponds to a half-period phase shift, $\psi=\pi$. The corresponding spatio-temporal symmetry subgroup is

$$
\Gamma_{\pi \sigma} \equiv\{I, \pi, \sigma, \pi \sigma\}
$$

Definition 2.10. A periodic orbit $O_{x}$ defined by a solution $x=\phi(t)$ of (6) has anti-phase symmetry if and only if at every point of the orbit

$$
\sigma \pi x=x, \quad \text { that is, } \quad \sigma \phi(t)=\phi\left(t+\frac{T}{2}\right) .
$$

For each element of $\Gamma_{\pi \sigma}$ applied to a periodic orbit $O_{x}$, there are two possibilities as in Lemma 2.1: either $O_{x}$ is mapped onto itself, or it is mapped to a disjoint periodic orbit. The elements $I$ and $\pi$ always map $O_{x}$ onto itself and cannot give a new orbit. If $O_{x}$ is the orbit corresponding to a periodic solution $x(t)$ and if $\sigma x(t)=x(t)$, then $x=[v, v]^{T}$ as before, and $O_{x}$ is an in-phase symmetric periodic orbit. If $\sigma \pi x(t)=x(t)$, then $x=[v, \pi v]^{T}$ and $O_{x}$ is an anti-phase symmetric periodic orbit. If $O_{x}$ is neither in-phase nor anti-phase symmetric, then $\sigma O_{x}$ and $O_{x}$ are disjoint periodic orbits; that is, such asymmetric periodic orbits exist in pairs. Thus, with only the Huygens permutation symmetry $\sigma$ and without the reflection symmetry $-I$, the discrete spatio-temporal symmetry (13) implies that if periodic orbits exist, they have either in-phase symmetry or anti-phase symmetry (as observed by Huygens), or they exist in pairs of periodic orbits with neither symmetry. In section 4, sufficient conditions for the existence of each of these three types of periodic orbits are given, and thus the escapement paradox is explained. If system (6) has the odd-Huygens symmetry group $\Gamma_{ \pm H}$ (5), then a stronger conclusion holds in the case $n=2$.

Theorem 2.11. Let $f$ be $\Gamma_{ \pm H}$-equivariant. Assume that $n=2$ and there exists a T-periodic orbit $O_{x} \subset \operatorname{Fix}\left(\Sigma_{-\sigma}\right)$ in (10) such that the origin $\mathbf{0}$ is in the interior of $O_{x}$. Then, on this periodic orbit, $\pi x=-x=\sigma x$; that is, anti-phase symmetry is the same as anti-permutation symmetry on such periodic orbits in $\operatorname{Fix}\left(\Sigma_{-\sigma}\right)$. 
Proof. Since $n=2, \operatorname{Fix}\left(\Sigma_{-\sigma}\right)$ is a 2-dimensional subspace, and by the Jordan curve theorem $O_{x}$ divides $\operatorname{Fix}\left(\Sigma_{-\sigma}\right)$ into disjoint interior and exterior regions, with $\mathbf{0}$ in the interior. By Lemma 2.1, either $O_{x}=O_{-x}$ or $O_{x} \cap O_{-x}=\emptyset$. But the latter case is excluded in two dimensions, since $\mathbf{0}$ must also lie in the interior of $O_{-x}$, forcing $O_{x}$ and $O_{-x}$ to intersect; therefore $O_{x}=O_{-x}$. Choosing any $x_{0} \in O_{x}$, then $-x_{0} \in O_{x}$ and there exists an $s \in[0, T]$ such that $\phi_{s}\left(x_{0}\right)=-x_{0}$. Now applying the reflection symmetry and using the last statement in Lemma 2.1, $\phi_{s}\left(-x_{0}\right)=-\left[-x_{0}\right]=x_{0}$ and $\phi_{s}\left(\phi_{s}\left(x_{0}\right)\right)=x_{0}$. Therefore, $s+s=T$, so $s=T / 2$, and $-x_{0}$ is a half-period away from $x_{0}$; that is, $\pi x=-x$ for any $x$. It is always true that $\sigma x=-x$ in $\operatorname{Fix}\left(\Sigma_{-\sigma}\right)$.

Theorem 2.11 is applied to solutions of the truncated normal form equations in section 3.4.

2.3. Semisimplicity of the Jacobian matrix. We assume the existence of a trivial equilibrium, $f(0)=0$ in (6), and suppose that $f$ is $\Gamma$-equivariant as in (1) for some $\Gamma$. Let $J=D_{x} f(0)$ be the Jacobian of the system (6) evaluated at $x=0$. Differentiating $f(\gamma x)=\gamma f(x)$ and evaluating at 0 gives

$$
J \gamma=\gamma J \quad \forall \gamma \in \Gamma .
$$

Therefore, if $f$ is $\Gamma$-equivariant, the Jacobian matrix $J \equiv D_{x} f(0)$ commutes with every element of $\Gamma$. The following is a well-known fact in group representation theory [13].

Lemma 2.12. If $A: \mathbb{R}^{m} \rightarrow \mathbb{R}^{m}$ is a linear map that commutes with $\Gamma \in \mathrm{GL}\left(\mathbb{R}^{m}\right)$, then

$$
A\left(W_{j}\right) \subseteq W_{j}
$$

for each component $W_{j}$ in the isotypic decomposition (7) of $\mathbb{R}^{m}$.

Assuming the Huygens symmetry $\Gamma=\Gamma_{H}$ for the isotypic decomposition (9), it follows that

$$
J\left(W_{+}\right) \subseteq W_{+} \quad \text { and } \quad J\left(W_{-}\right) \subseteq W_{-} .
$$

Rewrite $J$ as

$$
J=\left[\begin{array}{ll}
J_{1} & J_{2} \\
J_{3} & J_{4}
\end{array}\right]
$$

where $J_{k}, k=1,2,3,4$, are $n \times n$ real matrices. The fact that $J$ commutes with $\Gamma_{H}$ places a restriction on the form of $J$.

Lemma 2.13. $J \sigma=\sigma J$ if and only if $J_{1}=J_{4}$ and $J_{2}=J_{3}$; that is, $J$ has the form

$$
J=\left[\begin{array}{ll}
J_{1} & J_{2} \\
J_{2} & J_{1}
\end{array}\right] .
$$

Proof. The proof is elementary, by direct calculation of $J \sigma$ and $\sigma J$; see [22].

Any matrix such as $J$ that commutes with $\Gamma_{H}$ takes a simpler form if we change to a basis for $\mathbb{R}^{2 n}$ that respects the isotypic decomposition (9). Choose as a new basis for $\mathbb{R}^{2 n}$ the columns of the orthonormal matrix

$$
Q=\frac{1}{\sqrt{2}}\left[\begin{array}{rr}
I_{n} & I_{n} \\
I_{n} & -I_{n}
\end{array}\right] .
$$


The first $n$ columns of $Q$ are a basis of $W_{+}$, and the last $n$ for $W_{-}$. Then the Jacobian $J$ transforms to block diagonal form,

$$
\tilde{J}=Q J Q=\left[\begin{array}{cc}
J_{1}+J_{2} & O \\
O & J_{1}-J_{2}
\end{array}\right]
$$

where $J_{1}, J_{2}$ are as in (16). It follows that the set of eigenvalues of $J$ is the union of the sets of eigenvalues of $J_{1}+J_{2}$ and $J_{1}-J_{2}$. This leads immediately to an important fact about semisimple eigenvalues of any matrix such as $J$ that commutes with $\Gamma_{H}$. Since $\Gamma_{H}$ places no restriction on the individual submatrices $J_{1}+J_{2}$ and $J_{1}-J_{2}$, their eigenvalues will behave generically; that is, if a multiple eigenvalue exists in either one of $J_{1}+J_{2}$ or $J_{1}-J_{2}$, then generically it is nonsemisimple. However, if there exists a double eigenvalue that appears exactly once in each of $J_{1}+J_{2}$ and $J_{1}-J_{2}$, then it must be semisimple because it will have two independent eigenvectors, one in each of $W_{+}$and $W_{-}$by (15). This conclusion holds whether the eigenvalue is real or occurs in a complex conjugate pair. In particular, if $n=2$ and $J$ has double complex eigenvalues (as required for double Hopf bifurcation), then the complex conjugate pair must occur exactly once in each of $J_{1}+J_{2}$ and $J_{1}-J_{2}$, and the latter are forced to be semisimple. Thus we have proven the following theorem for $\Gamma_{H}$-equivariant systems.

Theorem 2.14. If the Jacobian matrix $J$ has complex eigenvalues and $n=2$, then $J$ is semisimple.

This model-independent result leads one to anticipate that the normal form for double Hopf bifurcation with Huygens symmetry will be very different from that of the generic 1:1 resonant Hopf bifurcation which has nonsemisimple eigenvalues [15, 35, 37].

3. Elphick-Huygens normal form. In this section we derive the Elphick normal form for double Hopf bifurcation at 1:1 resonance with Huygens symmetry, which we call the ElphickHuygens normal form. The Elphick normal form is equivalent to the classical Poincaré-Dulac normal form, which consists of "resonant monomials" that exist in some complement of the range of the homological operator. The advantages of the Elphick normal form for the present case are twofold: the Elphick normal form is unique, while the Poincaré normal form is not, and the Elphick normal form is determined by the property that it commutes with the Elphick symmetry group (25), which makes it possible to treat it and the Huygens symmetry on the same footing; see (26) in section 3.2.

Double Hopf bifurcation means that the Jacobian matrix $J=D_{x} f(0)$ has two pairs of complex conjugate purely imaginary eigenvalues. We assume henceforth that $J$ has no other eigenvalues that are on the imaginary axis or zero. In this case, the system has a 4-dimensional center manifold, and this manifold is flow-invariant by the center manifold theorem. There is a standard procedure [25] to reduce the system to this center manifold (at least locally and up to a polynomial truncation), and we assume this has been done. Therefore, in this section we can assume that the system of coupled oscillators is 4-dimensional; that is, each oscillator has dimension $n=2$, and then $J$ is semisimple by Theorem 2.14 .

3.1. Transformation to complex eigenbasis. For 1:1 resonant double Hopf bifurcation, the Jacobian matrix (17) of system (6) in $\mathbb{R}^{4}$ has a double complex conjugate pair of eigenvalues, $\lambda, \bar{\lambda}$, and four independent complex eigenvectors by Theorem 2.14. These eigenvectors 
are easily seen [22] to be the columns of an invertible matrix $P$ of the form

$$
P=\left[\begin{array}{cccc}
y_{1} & y_{2} & \bar{y}_{1} & \bar{y}_{2} \\
y_{1} & -y_{2} & \bar{y}_{1} & -\bar{y}_{2}
\end{array}\right], \quad y_{1}, y_{2} \in \mathbb{C}^{2},
$$

where $y_{1}$ and $y_{2}$ satisfy

$$
\left(J_{1}+J_{2}\right) y_{1}=\lambda y_{1}, \quad\left(J_{1}-J_{2}\right) y_{2}=\lambda y_{2} .
$$

Direct calculation shows that the third and fourth rows of $P^{-1}$ are the complex conjugates of the first and second rows, respectively. The transformation from real to complex coordinates is

$$
x=P z \quad \text { or } \quad z=P^{-1} x, \quad z \in \mathbb{C}^{4}, x \in \mathbb{R}^{4} .
$$

The real system $\dot{x}=f(x)$ transforms to the complex system

$$
\dot{z}=P^{-1} f(P z) \equiv g(z) .
$$

The form of $P^{-1}$ implies that $z_{3}=\overline{z_{1}}$ and $z_{4}=\overline{z_{2}}$, and the third and fourth equations of (21) are simply the conjugates of the first two; therefore, it suffices to write only the first two equations.

The Jacobian matrix at 1:1 resonance transforms in the complex coordinates $\mathbb{C}^{4}$ to

$$
\hat{J}=P^{-1} J P=\left[\begin{array}{cccc}
i \omega & 0 & 0 & 0 \\
0 & i \omega & 0 & 0 \\
0 & 0 & -i \omega & 0 \\
0 & 0 & 0 & -i \omega
\end{array}\right]
$$

where $\pm i \omega$ are the double purely imaginary complex eigenvalues, $\omega>0$. In the following, the hat on $\hat{J}$ is dropped, as it will be clear from the context whether $J$ is real or complex.

The representation of the Huygens symmetry group in the complex coordinates is determined as follows. If $\sigma$ represents the original Huygens permutation symmetry in the $x$ coordinates, then in the transformed coordinates $\sigma$ is represented by

$$
\kappa=P^{-1} \sigma P=\left[\begin{array}{cccc}
1 & 0 & 0 & 0 \\
0 & -1 & 0 & 0 \\
0 & 0 & 1 & 0 \\
0 & 0 & 0 & -1
\end{array}\right]
$$

by direct calculation with $P$ as in (20) [22]. Therefore the representation of the Huygens symmetry group in the space $\mathbb{C}^{4}$ of complex coordinates is

$$
\Gamma_{H}=\{I, \kappa\} .
$$


3.2. Calculation of the Elphick-Huygens normal form. The equivariant normal form in the complex coordinates of section 3.1 has been calculated in [22] by the method of Elphick et al. [10]. The key idea of this method is that the normal form is made unique by the condition that the nonlinear terms commute with the Elphick symmetry group,

$$
\Gamma_{E}=\left\{e^{J^{*} t}, t \in \mathbb{R}\right\}
$$

in addition to commuting with $\Gamma_{H}$. Here $J^{*}$ is the adjoint (conjugate transpose) of the Jacobian matrix. With $J$ given by $(22)$, it is clear that $\Gamma_{E}$ is a representation of the circle group $S^{1}$. Then the full group of symmetries of the equivariant normal form is

$$
\Gamma=\Gamma_{E} \times \Gamma_{H}
$$

It is shown in [10] that the condition of commuting with $e^{J^{*} t}$ is equivalent to satisfying a set of PDEs, which can be solved by the method of characteristics as follows.

Write the complex system (21) in the form

$$
\dot{z}=g(z)=J z+F(z), \quad z \in \mathbb{C}^{4},
$$

where $J$ is the Jacobian and $F: \mathbb{C}^{4} \rightarrow \mathbb{C}^{4}$ is the nonlinear part of the vector field. Then $F$ is in Elphick normal form if $F$ satisfies the PDE system

$$
D F(z) J^{*} z-J^{*} F(z)=0 .
$$

Writing (28) in components gives

$$
\sum_{j, l=1}^{4} \frac{\partial F_{i}}{\partial z_{j}} \bar{J}_{l j} z_{l}-\sum_{j=1}^{4} \bar{J}_{j i} F_{j}=0, \quad i=1, \ldots, 4 .
$$

The characteristic system associated with (29) is

$$
\frac{d z_{j}}{\sum_{l} \bar{J}_{l j} z_{l}}=\frac{d F_{i}}{\sum_{l} \bar{J}_{l i} F_{l}}, \quad i, j=1, \ldots, 4 .
$$

For the present case of 1:1 resonant Hopf bifurcation, $J$ given in (22) is diagonal, and $J^{*}=\bar{J}$. The Elphick symmetry group $\left\{e^{J^{*} t} \mid t \in \mathbb{R}\right\}$ is a representation of the compact Lie group $S^{1}$ on $\mathbb{C}^{4}$. Since the third and fourth equations in (27) are conjugates of the first two, it suffices to consider only the first two and solve for the two components $F_{1}$ and $F_{2}$. Using (22), equation (30) for $F_{1}$ simplifies to

$$
\frac{d z_{1}}{-i \omega z_{1}}=\frac{d z_{2}}{-i \omega z_{2}}=\frac{d \bar{z}_{1}}{i \omega \bar{z}_{1}}=\frac{d \bar{z}_{2}}{i \omega \bar{z}_{2}}=\frac{d F_{1}}{-i \omega F_{1}} .
$$

Similarly, for $F_{2}$, using the matrix (22), the characteristic system is

$$
\frac{d z_{1}}{-i \omega z_{1}}=\frac{d z_{2}}{-i \omega z_{2}}=\frac{d \bar{z}_{1}}{i \omega \bar{z}_{1}}=\frac{d \bar{z}_{2}}{i \omega \bar{z}_{2}}=\frac{d F_{2}}{-i \omega F_{2}} .
$$


Combining (31) and (32) and canceling iw yields

$$
\frac{d z_{1}}{-z_{1}}=\frac{d z_{2}}{-z_{2}}=\frac{d \bar{z}_{1}}{\bar{z}_{1}}=\frac{d \bar{z}_{2}}{\bar{z}_{2}}=\frac{d F_{1}}{-F_{1}}=\frac{d F_{2}}{-F_{2}}
$$

For (33) we find the following first integrals:

$$
\begin{aligned}
& \frac{d z_{1}}{-z_{1}}=\frac{d \bar{z}_{1}}{\bar{z}_{1}} \Longrightarrow z_{1} \bar{z}_{1}=C_{1}, \quad \frac{d z_{2}}{-z_{2}}=\frac{d \bar{z}_{2}}{\bar{z}_{2}} \Longrightarrow z_{2} \bar{z}_{2}=C_{2}, \quad \frac{d z_{2}}{-z_{2}}=\frac{d \bar{z}_{1}}{\bar{z}_{1}} \Longrightarrow \bar{z}_{1} z_{2}=C_{3}, \\
& \frac{d z_{1}}{-z_{1}}=\frac{d \bar{z}_{2}}{\bar{z}_{2}} \Longrightarrow z_{1} \bar{z}_{2}=C_{4}, \quad \frac{d z_{1}}{-z_{1}}=\frac{d F_{1}}{-F_{1}} \Longrightarrow \frac{F_{1}}{z_{1}}=C_{5}, \quad \frac{d z_{2}}{-z_{2}}=\frac{d F_{2}}{-F_{2}} \Longrightarrow \frac{F_{2}}{z_{2}}=C_{6} .
\end{aligned}
$$

These first integrals are enough to write the general solution for $F_{1}$ and $F_{2}$,

$$
\begin{aligned}
& F_{1}=z_{1} \psi_{1}\left(z_{1} \bar{z}_{1}, z_{2} \bar{z}_{2}, \bar{z}_{1} z_{2}, z_{1} \bar{z}_{2}\right), \\
& F_{2}=z_{2} \psi_{2}\left(z_{1} \bar{z}_{1}, z_{2} \bar{z}_{2}, \bar{z}_{1} z_{2}, z_{1} \bar{z}_{2}\right),
\end{aligned}
$$

where $\psi_{1}$ and $\psi_{2}$ are rational functions of a special type, since $F_{1}$ and $F_{2}$ must be polynomials. For $\psi_{1}$ the only possible rational terms are $\frac{z_{2} \bar{z}_{2}}{z_{1} \bar{z}_{2}}$ and $\frac{z_{2} \bar{z}_{1}}{z_{1} \bar{z}_{1}}$, each times some polynomial, because only in this case can we get a polynomial after multiplication by $z_{1}$. So $z_{1} \psi_{1}=z_{1} \varphi_{1}+z_{2} \varphi_{2}$, where $\varphi_{1}$ and $\varphi_{2}$ are polynomials of the variables $z_{1} \bar{z}_{1}, z_{2} \bar{z}_{2}, \bar{z}_{1} z_{2}, z_{1} \bar{z}_{2}$. A similar argument is applied for $\psi_{2}$. So the normal form for 1:1 resonance with Elphick symmetry in complex coordinates is

$$
\begin{aligned}
& \dot{z}_{1}=i \omega z_{1}+z_{1} \varphi_{1}\left(z_{1} \bar{z}_{1}, z_{2} \bar{z}_{2}, z_{1} \bar{z}_{2}, \bar{z}_{1} z_{2}\right)+z_{2} \varphi_{2}\left(z_{1} \bar{z}_{1}, z_{2} \bar{z}_{2}, z_{1} \bar{z}_{2}, \bar{z}_{1} z_{2}\right), \\
& \dot{z}_{2}=i \omega z_{2}+z_{1} \varphi_{3}\left(z_{1} \bar{z}_{1}, z_{2} \bar{z}_{2}, z_{1} \bar{z}_{2}, \bar{z}_{1} z_{2}\right)+z_{2} \varphi_{4}\left(z_{1} \bar{z}_{1}, z_{2} \bar{z}_{2}, z_{1} \bar{z}_{2}, \bar{z}_{1} z_{2}\right),
\end{aligned}
$$

where $\varphi_{1}, \ldots, \varphi_{4}$ are complex polynomials in their arguments.

In addition to the Elphick symmetry, the normal form must satisfy Huygens symmetry $\Gamma_{H}=\{I, \sigma\}$, where in complex coordinates $\sigma$ takes the form $\kappa$ given in (23). For (35) to commute with $\kappa$, the functions $\varphi_{i}$ must satisfy

$$
\varphi_{i}\left(z_{1} \bar{z}_{1}, z_{2} \bar{z}_{2},-z_{1} \bar{z}_{2},-\bar{z}_{1} z_{2}\right)=-\varphi_{i}\left(z_{1} \bar{z}_{1}, z_{2} \bar{z}_{2}, z_{1} \bar{z}_{2}, \bar{z}_{1} z_{2}\right), \quad i=2,3
$$

and

$$
\varphi_{i}\left(z_{1} \bar{z}_{1}, z_{2} \bar{z}_{2},-z_{1} \bar{z}_{2},-\bar{z}_{1} z_{2}\right)=\varphi_{i}\left(z_{1} \bar{z}_{1}, z_{2} \bar{z}_{2}, z_{1} \bar{z}_{2}, \bar{z}_{1} z_{2}\right), \quad i=1,4 .
$$

This further restricts (35) to the Elphick normal form with Huygens symmetry (see [22]),

$$
\begin{aligned}
& \dot{z}_{1}=i \omega z_{1}+z_{1} \cdot P_{1}\left(z_{1} \bar{z}_{1}, z_{2} \bar{z}_{2},\left[z_{1} \bar{z}_{2}\right]^{2},\left[\bar{z}_{1} z_{2}\right]^{2}\right)+\bar{z}_{1} z_{2}^{2} \cdot P_{2}\left(z_{1} \bar{z}_{1}, z_{2} \bar{z}_{2},\left[z_{1} \bar{z}_{2}\right]^{2},\left[\bar{z}_{1} z_{2}\right]^{2}\right), \\
& \dot{z}_{2}=i \omega z_{2}+z_{2} \cdot Q_{1}\left(z_{1} \bar{z}_{1}, z_{2} \bar{z}_{2},\left[z_{1} \bar{z}_{2}\right]^{2},\left[\bar{z}_{1} z_{2}\right]^{2}\right)+z_{1}^{2} \bar{z}_{2} \cdot Q_{2}\left(z_{1} \bar{z}_{1}, z_{2} \bar{z}_{2},\left[z_{1} \bar{z}_{2}\right]^{2},\left[\bar{z}_{1} z_{2}\right]^{2}\right)
\end{aligned}
$$

where $P_{1}, P_{2}, Q_{1}, Q_{2}$ are complex polynomials in their arguments. We call (36) the ElphickHuygens normal form. It is assumed that the constant terms $P_{1}(\mathbf{0})$ and $Q_{1}(\mathbf{0})$ in (36) are zero, because they can affect only the linear part of the system and the linear terms already are given uniquely by the Jacobian $J$. They determine the linear unfolding parameters in the 
next section. An alternative derivation of this equivariant normal form is presented in [22], using the Hilbert-Weyl theorem. The result is the same as (36).

The behavior of the system is often easier to visualize if the equations are written in polar coordinates rather than complex coordinates. Let

$$
z_{1}=r_{1} e^{i \theta_{1}} \quad \text { and } \quad z_{2}=r_{2} e^{i \theta_{2}}, \quad r_{1}, r_{2} \geq 0 .
$$

Substituting $\dot{z}_{1}=\dot{r}_{1} e^{i \theta_{1}}+i r_{1} \dot{\theta}_{1} e^{i \theta_{1}}, \dot{z}_{2}=\dot{r}_{2} e^{i \theta_{2}}+i r_{2} \dot{\theta}_{2} e^{i \theta_{2}}$, and separating the real and imaginary parts, puts (36) in polar coordinates:

$$
\begin{aligned}
& \dot{r}_{1}=r_{1}\left\{\operatorname{Re}\left(P_{1}\right)+r_{2}^{2}\left[\operatorname{Re}\left(P_{2}\right) \cos 2\left(\theta_{1}-\theta_{2}\right)+\operatorname{Im}\left(P_{2}\right) \sin 2\left(\theta_{1}-\theta_{2}\right)\right]\right\}, \\
& \dot{r}_{2}=r_{2}\left\{\operatorname{Re}\left(Q_{1}\right)+r_{1}^{2}\left[\operatorname{Re}\left(Q_{2}\right) \cos 2\left(\theta_{1}-\theta_{2}\right)-\operatorname{Im}\left(Q_{2}\right) \sin 2\left(\theta_{1}-\theta_{2}\right)\right]\right\}, \\
& \dot{\theta_{1}}=\omega+\operatorname{Im}\left(P_{1}\right)+r_{2}^{2}\left[\operatorname{Im}\left(P_{2}\right) \cos 2\left(\theta_{1}-\theta_{2}\right)-\operatorname{Re}\left(P_{2}\right) \sin 2\left(\theta_{1}-\theta_{2}\right)\right], \\
& \dot{\theta}_{2}=\omega+\operatorname{Im}\left(Q_{1}\right)+r_{1}^{2}\left[\operatorname{Im}\left(Q_{2}\right) \cos 2\left(\theta_{1}-\theta_{2}\right)+\operatorname{Re}\left(Q_{2}\right) \sin 2\left(\theta_{1}-\theta_{2}\right)\right] .
\end{aligned}
$$

Here $P_{1}, P_{2}, Q_{1}, Q_{2}$ are complex-valued functions of $r_{1}^{2}, r_{2}^{2}, r_{1}^{2} r_{2}^{2} e^{2 i\left(\theta_{1}-\theta_{2}\right)}$, and $r_{1}^{2} r_{2}^{2} e^{-2 i\left(\theta_{1}-\theta_{2}\right)}$. Since the angle coordinates $\theta_{1}, \theta_{2}$ appear in (38) only as the difference $\theta_{1}-\theta_{2}$, the number of equations and of variables can be reduced from 4 to 3 , by subtracting the last two equations and substituting $\theta \equiv 2\left(\theta_{1}-\theta_{2}\right)$. Then (38) becomes

$$
\begin{aligned}
& \dot{r}_{1}=r_{1}\left\{\operatorname{Re}\left(P_{1}\right)+r_{2}^{2}\left[\operatorname{Re}\left(P_{2}\right) \cos \theta+\operatorname{Im}\left(P_{2}\right) \sin \theta\right]\right\}, \\
& \dot{r}_{2}=r_{2}\left\{\operatorname{Re}\left(Q_{1}\right)+r_{1}^{2}\left[\operatorname{Re}\left(Q_{2}\right) \cos \theta-\operatorname{Im}\left(Q_{2}\right) \sin \theta\right]\right\}, \\
& \dot{\theta}=2\{ \operatorname{Im}\left(P_{1}\right)-\operatorname{Im}\left(Q_{1}\right)-r_{1}^{2}\left[\operatorname{Im}\left(Q_{2}\right) \cos \theta+\operatorname{Re}\left(Q_{2}\right) \sin \theta\right] \\
&\left.+r_{2}^{2}\left[\operatorname{Im}\left(P_{2}\right) \cos \theta-\operatorname{Re}\left(P_{2}\right) \sin \theta\right]\right\} .
\end{aligned}
$$

We call this the Elphick-Huygens normal form in polar coordinates.

3.3. Unfolding parameters. In order to study the behavior of systems not only at, but also near, 1:1 resonant Hopf bifurcation (while preserving the Huygens symmetry), we need to include unfolding parameters in the normal form. The unfolding parameters may be calculated by either the method of Elphick et al. or by the Hilbert-Weyl method; both have been carried out in [22].

We assume that the nonlinear terms in the normal form are generic; that is, the only degeneracy in the system (locally) is in the Jacobian. The simplest way to determine the unfolding parameters is to consider the most general linear terms that are allowed to appear in the system, while satisfying both the Elphick and Huygens symmetries. These are given by the constant terms $P_{1}(\mathbf{0}) \equiv a_{0}=\alpha_{0}+i \gamma_{0}$ and $Q_{1}(\mathbf{0}) \equiv c_{0}=\zeta_{0}+i \eta_{0}$, of the complex polynomials $P_{1}$ and $Q_{1}$ respectively, in (36). These are four real parameters, so the codimension of the bifurcation is at most four. The two real parts $\alpha_{0}, \zeta_{0}$ correspond to the real parts of the two purely imaginary eigenvalues, so both are needed to unfold the double Hopf bifurcation. For the two imaginary parts $\gamma_{0}, \eta_{0}$ only the difference is important; this corresponds to splitting the 1:1 resonant eigenvalues on the imaginary axis. They may be combined into one parameter without loss of generality, leaving just three unfolding parameters, which we write as

$$
\mu_{1}=\alpha_{0}, \quad \mu_{2}=\zeta_{0}, \quad \text { and } \mu_{3}=\gamma_{0}-\eta_{0} .
$$


Then the codimension of this bifurcation is three, and the Elphick-Huygens normal form (39) in polar coordinates, with explicit unfolding parameters $\mu_{1}, \mu_{2}, \mu_{3}$, is

$$
\begin{aligned}
& \dot{r}_{1}=r_{1}\left\{\mu_{1}+\operatorname{Re}\left(P_{1}\right)+r_{2}^{2}\left[\operatorname{Re}\left(P_{2}\right) \cos \theta+\operatorname{Im}\left(P_{2}\right) \sin \theta\right]\right\}, \\
& \dot{r}_{2}=r_{2}\left\{\mu_{2}+\operatorname{Re}\left(Q_{1}\right)+r_{1}^{2}\left[\operatorname{Re}\left(Q_{2}\right) \cos \theta-\operatorname{Im}\left(Q_{2}\right) \sin \theta\right]\right\} \text {, } \\
& \dot{\theta}=2\left\{\mu_{3}+\operatorname{Im}\left(P_{1}\right)-\operatorname{Im}\left(Q_{1}\right)-r_{1}^{2}\left[\operatorname{Im}\left(Q_{2}\right) \cos \theta+\operatorname{Re}\left(Q_{2}\right) \sin \theta\right]\right. \\
& \left.+r_{2}^{2}\left[\operatorname{Im}\left(P_{2}\right) \cos \theta-\operatorname{Re}\left(P_{2}\right) \sin \theta\right]\right\},
\end{aligned}
$$

where, as in (36), the constant terms of $P_{1}$ and $Q_{1}$ are assumed equal to zero.

3.4. The escapement paradox revisited. As shown in section 2.2 , the ubiquitous observations of in-phase and anti-phase periodic oscillations in the literature, for systems with Huygens symmetry, can be explained in terms of the group of spatio-temporal symmetries $\Gamma_{\pi \sigma}$ (13). This is true whether the system has an internal reflection symmetry $f(-x)=-f(x)$ or breaks this symmetry, for example, with a unidirectional escapement mechanism. The Elphick normal form calculated here sheds further light on the escapement paradox.

The key to the determination of the Elphick normal form is that the nonlinear terms are required to commute with the Elphick symmetry group $\Gamma_{E}=\left\{e^{J^{*} t}\right\}$; see (25). But from (22), when $t=\frac{\pi}{\omega}$,

$$
e^{J^{*} t}=e^{ \pm i \pi} I=-I .
$$

Thus the reflection symmetry group $\{I,-I\}$ is a subgroup of the Elphick symmetry group, and the Elphick-Huygens normal form always has the odd-Huygens symmetry $\Gamma_{ \pm H}$. Therefore, the Elphick-Huygens normal form (36) has both in-phase and antipermutation invariant fixedpoint subspaces, corresponding to subgroups $\Sigma_{\sigma}$ and $\Sigma_{-\sigma}$ as in (5), even if the original system does not. Furthermore, since Theorem 2.11 applies to the truncated Elphick-Huygens normal form equations, small periodic orbits about 0 in $\operatorname{Fix}\left(\Sigma_{-\sigma}\right)$ satisfy $\pi x=-x=\sigma x$; that is, anti-phase symmetry is the same as antipermutation symmetry for periodic orbits.

This is not a model-independent result in the sense of section 2, since the Elphick symmetry is not a property of the original system, but it derives from the assumed double Hopf bifurcation and is a local analytical result valid only for the truncated normal form. It must be noted that the transformation to normal form in general leaves a remainder (or tail) that does not have the Elphick symmetry, and this remainder is omitted in (36). This remainder can be made arbitrarily small in a neighborhood of the bifurcation point. The transformation of coordinates between the original system and the normal form (36) is a sequence of nearidentity polynomial maps, which preserve topological features [16]. Therefore, sufficiently near to the double Hopf bifurcation, when the fixed-point subspace of antipermutation solutions is normally hyperbolic, the inverse image of this flow-invariant subspace under the normal form transformation generically approximates a normally hyperbolic invariant manifold.

Periodic solutions of (38), (39), or (40) have either in-phase or anti-phase symmetry, or else exist in distinct asymmetric pairs, as follows. The Huygens permutation symmetry $\sigma$ is represented by $\kappa$ defined by (23) in complex coordinates; that is, $\kappa\left(z_{1}, z_{2}\right)=\left(z_{1},-z_{2}\right)$. The fixed-point subspace corresponding to $\kappa$ is obviously $\left\{\left(z_{1}, 0\right)\right\}$; this is the previously noted 
invariant subspace of in-phase solutions. In polar coordinates, $\kappa\left(r_{1}, r_{2}, \theta_{1}, \theta_{2}\right)=\left(r_{1}, r_{2}, \theta_{1}, \theta_{2}+\right.$ $\pi$ ), from (37). For an anti-phase periodic solution, $r_{1}=0$, and

$$
\kappa \pi\left(0, r_{2}, 0, \theta_{2}\right)=\left(0, r_{2}, 0, \theta+\pi+\pi\right)=\left(0, r_{2}, 0, \theta_{2}\right),
$$

which confirms the anti-phase symmetry. Otherwise, if both $r_{1}>0$ and $r_{2}>0$, then

$$
\kappa \pi\left(r_{1}, r_{2}, \theta_{1}, \theta_{2}\right)=\left(r_{1}, r_{2}, \theta_{1}+\pi, \theta_{2}\right),
$$

which is a second orbit disjoint from $\left(r_{1}, r_{2}, \theta_{1}, \theta_{2}\right)$.

3.5. Cubic truncated system. For further work we write the normal form explicitly in terms of polynomials up to degree 3 . The first few terms of the polynomials $P_{1}, P_{2}, Q_{1}$, and $Q_{2}$ are

$$
\begin{aligned}
P_{1}\left(z_{1} \bar{z}_{1}, z_{2} \bar{z}_{2}, z_{1}^{2} \bar{z}_{2}^{2}, \bar{z}_{1}^{2} z_{2}^{2}\right)= & a_{0}+a_{1} z_{1} \bar{z}_{1}+a_{2} z_{2} \bar{z}_{2}+a_{3} z_{1}^{2} \bar{z}_{2}^{2}+a_{4} \bar{z}_{1}^{2} z_{2}^{2} \\
& +a_{5}\left(z_{1} \bar{z}_{1}\right)^{2}+a_{6}\left(z_{2} \bar{z}_{2}\right)^{2}+a_{7} z_{1} \bar{z}_{1} z_{2} \bar{z}_{2}+\cdots, \\
P_{2}\left(z_{1} \bar{z}_{1}, z_{2} \bar{z}_{2}, z_{1}^{2} \bar{z}_{2}^{2}, \bar{z}_{1}^{2} z_{2}^{2}\right)= & b_{0}+b_{1} z_{1} \bar{z}_{1}+b_{2} z_{2} \bar{z}_{2}+\cdots, \\
Q_{1}\left(z_{1} \bar{z}_{1}, z_{2} \bar{z}_{2}, z_{1}^{2} \bar{z}_{2}^{2}, \bar{z}_{1}^{2} z_{2}^{2}\right)= & c_{0}+c_{1} z_{1} \bar{z}_{1}+c_{2} z_{2} \bar{z}_{2}+c_{3} z_{1}^{2} \bar{z}_{2}^{2}+c_{4} \bar{z}_{1}^{2} z_{2}^{2} \\
& +c_{5}\left(z_{1} \bar{z}_{1}\right)^{2}+c_{6}\left(z_{2} \bar{z}_{2}\right)^{2}+c_{7} z_{1} \bar{z}_{1} z_{2} \bar{z}_{2}+\cdots, \\
Q_{2}\left(z_{1} \bar{z}_{1}, z_{2} \bar{z}_{2}, z_{1}^{2} \bar{z}_{2}^{2}, \bar{z}_{1}^{2} z_{2}^{2}\right)= & d_{0}+d_{1} z_{1} \bar{z}_{1}+d_{2} z_{2} \bar{z}_{2}+\cdots .
\end{aligned}
$$

Since the coefficients of the polynomials are complex numbers, let us introduce the following real notations:

$$
a_{k}=\alpha_{k}+i \gamma_{k}, \quad b_{k}=\beta_{k}+i \delta_{k}, \quad c_{k}=\zeta_{k}+i \eta_{k}, \quad d_{k}=\kappa_{k}+i \nu_{k}
$$

As in (40), the constant terms $a_{0}$ and $c_{0}$ of the polynomials $P_{1}$ and $Q_{1}$ correspond to the unfolding parameters. Then in the polar variables (37), after writing $e^{i \theta}=\cos \theta+i \sin \theta$ and separating real and imaginary parts [22], the normal form equations (39) up to degree 3 are

$$
\begin{aligned}
\dot{r}_{1} & =r_{1}\left[\mu_{1}+\alpha_{1} r_{1}^{2}+\left(\alpha_{2}+\beta_{0} \cos \theta+\delta_{0} \sin \theta\right) r_{2}^{2}\right], \\
\dot{r}_{2} & =r_{2}\left[\mu_{2}+\left(\zeta_{1}+\kappa_{0} \cos \theta-\nu_{0} \sin \theta\right) r_{1}^{2}+\zeta_{2} r_{2}^{2}\right], \\
\dot{\theta} & =2\left[\mu_{3}+\left(\gamma_{1}-\eta_{1}-\nu_{0} \cos \theta-\kappa_{0} \sin \theta\right) r_{1}^{2}+\left(\gamma_{2}-\eta_{2}+\delta_{0} \cos \theta-\beta_{0} \sin \theta\right) r_{2}^{2}\right] .
\end{aligned}
$$

We can simplify this system by writing the sum involving $\cos \theta$ and $\sin \theta$ as a $\operatorname{single}$ trigonometric function, as follows. For the expression in the first equation, use the identity

$$
\beta_{0} \cos \theta+\delta_{0} \sin \theta=\sqrt{\beta_{0}^{2}+\delta_{0}^{2}}\left(\frac{\beta_{0}}{\sqrt{\beta_{0}^{2}+\delta_{0}^{2}}} \cos \theta+\frac{\delta_{0}}{\sqrt{\beta_{0}^{2}+\delta_{0}^{2}}} \sin \theta\right) .
$$

Then set $A_{1}=\sqrt{\beta_{0}^{2}+\delta_{0}^{2}} \geq 0, \cos \phi_{1}=\frac{\beta_{0}}{\sqrt{\beta_{0}^{2}+\delta_{0}^{2}}}$, and $\sin \phi_{1}=\frac{\delta_{0}}{\sqrt{\beta_{0}^{2}+\delta_{0}^{2}}}$, and obtain

$$
\beta_{0} \cos \theta+\delta_{0} \sin \theta=A_{1}\left(\cos \theta \cos \phi_{1}+\sin \theta \sin \phi_{1}\right)=A_{1} \cos \left(\theta-\phi_{1}\right) .
$$


In a similar way [22], we replace the other trigonometric sums in (43). Then (43) takes the form

$$
\begin{aligned}
\dot{r}_{1} & =r_{1}\left[\mu_{1}+\alpha_{1} r_{1}^{2}+\left(\alpha_{2}+A_{1} \cos \left(\theta-\phi_{1}\right)\right) r_{2}^{2}\right], \\
\dot{r}_{2} & =r_{2}\left[\mu_{2}+\left(\zeta_{1}+A_{2} \cos \left(\theta-\phi_{2}\right)\right) r_{1}^{2}+\zeta_{2} r_{2}^{2}\right], \\
\dot{\theta} & =2\left[\mu_{3}+\left(\gamma_{1}-\eta_{1}-A_{2} \sin \left(\theta-\phi_{2}\right)\right) r_{1}^{2}+\left(\gamma_{2}-\eta_{2}-A_{1} \sin \left(\theta-\phi_{1}\right)\right) r_{2}^{2}\right] .
\end{aligned}
$$

Note that the coefficients $A_{1}$ and $A_{2}$ defined here are nonnegative, by construction. To simplify the notation further in (44), relabel the coefficients $\alpha_{1}=a_{11}, \alpha_{2}=a_{12}, \zeta_{1}=a_{21}, \zeta_{2}=$ $a_{22}, \gamma_{1}-\eta_{1}=a_{31}$, and $\gamma_{2}-\eta_{2}=a_{32}$. Also, define $\hat{\theta}$ and $\phi$ by $\hat{\theta}=\theta-\phi_{1}, \phi=\phi_{1}-\phi_{2}$, substitute, and drop the hats; then (44) simplifies to

$$
\begin{aligned}
\dot{r}_{1} & =r_{1}\left[\mu_{1}+a_{11} r_{1}^{2}+\left(a_{12}+A_{1} \cos \theta\right) r_{2}^{2}\right], \\
\dot{r}_{2} & =r_{2}\left[\mu_{2}+\left(a_{21}+A_{2} \cos (\theta+\phi)\right) r_{1}^{2}+a_{22} r_{2}^{2}\right], \\
\dot{\theta} & =2\left[\mu_{3}+\left(a_{31}-A_{2} \sin (\theta+\phi)\right) r_{1}^{2}+\left(a_{32}-A_{1} \sin \theta\right) r_{2}^{2}\right] .
\end{aligned}
$$

System (45) is the main object of study in sections 4,5 , and 6 .

3.6. Comparison with nonresonant double Hopf bifurcation. It is instructive to compare (45) to the normal form for the classical case of nonresonant double Hopf bifurcation; see $[16,25,38]$. To cubic order, the normal form for that case can be written

$$
\begin{aligned}
\dot{r}_{1} & =r_{1}\left[\mu_{1}+a_{11} r_{1}^{2}+a_{12} r_{2}^{2}\right], \\
\dot{r}_{2} & =r_{2}\left[\mu_{2}+a_{21} r_{1}^{2}+a_{22} r_{2}^{2}\right], \\
\dot{\theta} & =2\left[\mu_{3}+a_{31} r_{1}^{2}+a_{32} r_{2}^{2}\right] .
\end{aligned}
$$

These equations have the same form as the cubic truncated normal form equations of this study if one sets $A_{1}=A_{2}=0$ in (45). The only other difference is that in this paper $\mu_{3}$ is a "small parameter" that can be arbitrarily close to zero, whereas, in the case of nonresonant double Hopf bifurcation, $\mu_{3} \equiv \omega_{1}-\omega_{2}$, the difference in the natural frequencies, which is of order one.

An equilibrium point $\left(r_{10}, r_{20}\right) \neq(0,0)$ of the first two of these equations is given by

$$
r_{10}^{2}=\frac{-\mu_{1} a_{22}+\mu_{2} a_{12}}{a_{11} a_{22}-a_{12} a_{21}}, \quad r_{20}^{2}=\frac{\mu_{1} a_{21}-\mu_{2} a_{11}}{a_{11} a_{22}-a_{12} a_{21}},
$$

provided these expressions are positive. Then substituting (47) into the third equation in (46) gives

$$
\theta(t)=2\left[\mu_{3}+a_{31} r_{10}^{2}+a_{32} r_{20}^{2}\right] t+\theta_{0} .
$$

In this case the absolute phase difference between the two oscillations increases (or decreases) monotonically in $t$, so generically this represents a quasi-periodic orbit on a 2 -torus. This suggests that there is a limiting case in which the known structurally stable results for the nonresonant double Hopf bifurcation, as determined by (46), carry over to the present case. This is reasonable, since the Jacobian $J$ is semisimple, and in every neighbourhood of the bifurcation point of 1:1 resonance there are nonresonant double Hopf bifurcations with frequency ratio $\omega_{1}: \omega_{2}$ irrational (i.e., nonresonant). Quasi-periodic solutions are studied in section 5 . 
3.7. Supercritical case. Assuming the coefficients $a_{11}$ and $a_{22}$ are nonzero, the 3 -dimensional normal form (45) can be further simplified by rescaling the variables. Let

$$
\hat{r}_{1}=\sqrt{\left|a_{11}\right|} r_{1}, \quad \hat{r}_{2}=\sqrt{\left|a_{22}\right|} r_{2} ;
$$

then substitute into (45) and drop the hats. This yields

$$
\begin{aligned}
\dot{r}_{1} & =r_{1}\left[\mu_{1}+s_{1} r_{1}^{2}+\left(b_{12}+B_{1} \cos \theta\right) r_{2}^{2}\right], \\
\dot{r}_{2} & =r_{2}\left[\mu_{2}+\left(b_{21}+B_{2} \cos (\theta+\phi)\right) r_{1}^{2}+s_{2} r_{2}^{2}\right], \\
\dot{\theta} & =2\left[\mu_{3}+\left(b_{31}-B_{2} \sin (\theta+\phi)\right) r_{1}^{2}+\left(b_{32}-B_{1} \sin \theta\right) r_{2}^{2}\right],
\end{aligned}
$$

where $s_{i}=\operatorname{sign}\left(a_{i i}\right)$, and the new coefficients are

$$
B_{1}=\frac{A_{1}}{\left|a_{22}\right|}, B_{2}=\frac{A_{2}}{\left|a_{11}\right|}, b_{12}=\frac{a_{12}}{\left|a_{22}\right|}, b_{21}=\frac{a_{21}}{\left|a_{11}\right|}, b_{31}=\frac{a_{31}}{\left|a_{11}\right|}, b_{32}=\frac{a_{32}}{\left|a_{22}\right|} .
$$

Note that the coefficients $B_{1}$ and $B_{2}$ are nonnegative because $A_{1}$ and $A_{2}$ are nonnegative.

In terms of the constants $s_{i}$, mathematically there are just two generic cases to consider. If both $s_{1}$ and $s_{2}$ are positive, then a time reversal (and reversal of stability properties) changes the system to one where both $s_{1}$ and $s_{2}$ are negative. If $s_{1}>0$ but $s_{2}<0$, then an exchange of all subscripts 1 and 2 in (48), along with an exchange of $\theta+\phi$ and $\theta$, yields a system with $s_{1}<0$ and $s_{2}>0$. Effectively this is just a switching of the in-phase and anti-phase roles. With these considerations, it is sufficient to assume $s_{1}=-1$ and consider the two cases $s_{2}= \pm 1$.

Applying the same transformations, truncation, and scalings to the 4-dimensional system (38), it becomes

$$
\begin{aligned}
& \dot{r}_{1}=r_{1}\left[\mu_{1}+s_{1} r_{1}^{2}+\left(b_{12}+B_{1} \cos \theta\right) r_{2}^{2}\right], \\
& \dot{r}_{2}=r_{2}\left[\mu_{2}+\left(b_{21}+B_{2} \cos (\theta+\phi)\right) r_{1}^{2}+s_{2} r_{2}^{2}\right], \\
& \dot{\theta}_{1}=\omega+\gamma_{0}+c_{11} r_{1}^{2}+\left[c_{12}-B_{1} \sin \theta\right] r_{2}^{2}, \\
& \dot{\theta}_{2}=\omega+\eta_{0}+\left[c_{21}+B_{2} \sin (\theta+\phi)\right] r_{1}^{2}+c_{22} r_{2}^{2},
\end{aligned}
$$

where

$$
c_{11}=\frac{\gamma_{1}}{\left|a_{11}\right|}, \quad c_{12}=\frac{\gamma_{2}}{\left|a_{22}\right|}, \quad c_{21}=\frac{\eta_{1}}{\left|a_{11}\right|}, \quad \text { and } \quad c_{22}=\frac{\eta_{2}}{\left|a_{22}\right|} .
$$

The following relations hold between the parameters of this system and the 3-dimensional system (48): $\mu_{3}=\gamma_{0}-\eta_{0}, b_{31}=c_{11}-c_{21}, b_{32}=c_{12}-c_{22}$.

In applications, stable solutions are of greatest interest. In section 4.1, it is shown that the normal mode in-phase and anti-phase solutions are stable (at least within their respective invariant subspaces) and arise as supercritical Hopf bifurcations if and only if

$$
s_{1}=-1 \quad \text { and } s_{2}=-1, \quad \text { that is, } a_{11}<0 \text { and } a_{22}<0 .
$$

If either of these coefficients is positive, then there is an unstable direction in the state space, with trajectories leaving a neighborhood of the origin. 
In sections 4,5 , and 6 , the supercritical case represented by the normal form,

$$
\begin{aligned}
\dot{r}_{1} & =r_{1}\left[\mu_{1}-r_{1}^{2}+\left(b_{12}+B_{1} \cos \theta\right) r_{2}^{2}\right], \\
\dot{r}_{2} & =r_{2}\left[\mu_{2}+\left(b_{21}+B_{2} \cos (\theta+\phi)\right) r_{1}^{2}-r_{2}^{2}\right], \\
\dot{\theta} & =2\left[\mu_{3}+\left(b_{31}-B_{2} \sin (\theta+\phi)\right) r_{1}^{2}+\left(b_{32}-B_{1} \sin \theta\right) r_{2}^{2}\right],
\end{aligned}
$$

is investigated in detail. The cases where one or both of $a_{11}$ and $a_{22}$ vanish (so that $s_{1}$ or $s_{2}$ is zero) are degenerate cases that we do not consider. The supercritical case for (50) is

$$
\begin{aligned}
& \dot{r}_{1}=r_{1}\left[\mu_{1}-r_{1}^{2}+\left(b_{12}+B_{1} \cos \theta\right) r_{2}^{2}\right], \\
& \dot{r}_{2}=r_{2}\left[\mu_{2}+\left(b_{21}+B_{2} \cos (\theta+\phi)\right) r_{1}^{2}-r_{2}^{2}\right], \\
& \dot{\theta}_{1}=\omega+\gamma_{0}+c_{11} r_{1}^{2}+\left[c_{12}-B_{1} \sin \theta\right] r_{2}^{2}, \\
& \dot{\theta}_{2}=\omega+\eta_{0}+\left[c_{21}+B_{2} \sin (\theta+\phi)\right] r_{1}^{2}+c_{22} r_{2}^{2} .
\end{aligned}
$$

4. Periodic solutions. This section presents a study of periodic solutions of the truncated Elphick-Huygens normal form. As predicted by the symmetry arguments of section 2.2, three distinct types of periodic orbits exist, for the normal form and for the original system. In section 4.1, we explore the existence of in-phase and anti-phase periodic solutions, known as normal modes. In section 4.2 we find periodic solutions that are neither in-phase nor antiphase but can be considered a phase-locked combination of the two. They occur in pairs and are called phase-locked mixed mode periodic solutions.

4.1. In-phase and anti-phase normal mode solutions. Consider the 4-dimensional normal form (50). There is always a trivial equilibrium solution at the origin. The invariant subspace of in-phase solutions in polar coordinates corresponds to $\left\{r_{1}>0, r_{2}=0\right\}$, and similarly the anti-phase invariant subspace is $\left\{r_{1}=0, r_{2}>0\right\}$. If one substitutes $r_{2}=0$ into (50), then the unfolded truncated Elphick-Huygens normal form reduces to

$$
\begin{aligned}
& \dot{r}_{1}=r_{1}\left[\mu_{1}+s_{1} r_{1}^{2}\right], \\
& \dot{\theta}_{1}=\omega+\gamma_{0}+c_{11} r_{1}^{2} .
\end{aligned}
$$

Note that (55) is the normal form for the classical Hopf bifurcation. Assume that the Hopf nondegeneracy condition $s_{1} \neq 0$ holds. Then it follows that, in the invariant subspace of in-phase solutions, there exists a 1-parameter family of in-phase periodic solutions, for small $\mu_{1}$ satisfying $\mu_{1} s_{1}<0$, of amplitude asymptotically near

$$
r_{1} \sim \sqrt{-\frac{\mu_{1}}{s_{1}}}
$$

Similarly, in the invariant subspace of anti-phase solutions $\left\{r_{1}=0, r_{2}>0\right\}$, there is a system of the same form as (55), and if $s_{2} \neq 0$, there exists a 1-parameter family of periodic anti-phase solutions, for small $\mu_{2}$ satisfying $\mu_{2} s_{2}<0$, of amplitude asymptotically near

$$
r_{2} \sim \sqrt{-\frac{\mu_{2}}{s_{2}}}
$$


We call each of these in-phase and anti-phase periodic oscillations normal mode solutions, and we call the two Hopf bifurcations from the trivial solution that give rise to these normal mode solutions primary bifurcations.

The stability of these normal mode solutions within their respective invariant subspaces is determined by Hopf's "exchange of stabilities" formula [12]. That is, if $s_{1}=-1$ (resp., $s_{2}=$ -1 ), then the Hopf bifurcation for the in-phase (resp., anti-phase) solutions is supercritical (exists for $\mu_{j}>0$ ), and the periodic orbit is asymptotically orbitally stable in its 2-dimensional invariant subspace. Otherwise, the bifurcation is subcritical and the orbit is unstable. If both bifurcations are supercritical, then either one or both may be stable in the full 4-dimensional state space of the Elphick-Huygens normal form depending on other nonlinear coefficients, as is the case for the nonresonant double Hopf bifurcation [16, 25, 38].

4.2. Phase-locked mixed mode periodic solutions. In this section, we study nontrivial equilibrium solutions of the 3-dimensional truncated Elphick-Huygens normal form (48). The state space of (48) is

$$
\mathcal{H}=\left\{\left(r_{1}, r_{2}, \theta\right) \mid r_{1} \geq 0, r_{2} \geq 0, \theta \in \mathbb{R}(\bmod 2 \pi)\right\} .
$$

We represent this as a semi-infinite slab of thickness $2 \pi$, with the top and bottom surfaces identified. A nontrivial equilibrium of (48) consists of constants $r_{10}>0, r_{20}>0$, and $\theta_{0} \in[0,2 \pi)$ satisfying

$$
\begin{aligned}
& 0=\mu_{1}+s_{1} r_{10}^{2}+\left(b_{12}+B_{1} \cos \theta_{0}\right) r_{20}^{2}, \\
& 0=\mu_{2}+\left(b_{21}+B_{2} \cos \left(\theta_{0}+\phi\right)\right) r_{10}^{2}+s_{2} r_{20}^{2}, \\
& 0=\mu_{3}+\left(b_{31}-B_{2} \sin \left(\theta_{0}+\phi\right)\right) r_{10}^{2}+\left(b_{32}-B_{1} \sin \theta_{0}\right) r_{20}^{2} .
\end{aligned}
$$

Given such an equilibrium $\left(r_{10}, r_{20}, \theta_{0}\right)$ of $(48)$, if it is hyperbolic, then by standard arguments locally there exists a nearby equilibrium of the nontruncated 3-dimensional normal form equations. In turn, this implies a periodic solution of the full 4-dimensional system as follows. Since both $r_{1}$ and $r_{2}$ are positive, the solution has nonzero components in both of the in-phase and anti-phase invariant subspaces. Therefore, we call it a mixed mode solution. Since $\dot{\theta}=0$, the phase difference $\theta / 2=\theta_{1}-\theta_{2}$ is constant; that is, this is a phase-locked periodic solution. Locally, $\theta_{1}$ and $\theta_{2}$ both increase monotonically and at the same rate. Such phase-locked mixed mode periodic solutions have been observed [5, 27]; the present analysis illuminates their origins.

If $\left(r_{10}, r_{20}, \theta_{0}\right)$ is an asymptotically stable equilibrium, then the corresponding mixed mode periodic orbit is orbitally asymptotically stable. As noted in section 2.2 and at the end of section 3.4, these mixed mode periodic orbits exist in disjoint pairs. The pairs correspond to permutation of the two identical oscillators and therefore are not fundamentally different.

The equations (57) are linear in $r_{10}^{2}, r_{20}^{2}$, and the first two are easily solved for each fixed $\theta$. The solution is

$$
\begin{aligned}
& r_{10}^{2}(\theta)=\frac{-\mu_{1} s_{2}+\mu_{2}\left(b_{12}+B_{1} \cos \theta\right)}{s_{1} s_{2}-\left(b_{12}+B_{1} \cos \theta\right)\left(b_{21}+B_{2} \cos (\theta+\phi)\right)}, \\
& r_{20}^{2}(\theta)=\frac{-\mu_{2} s_{1}+\mu_{1}\left(b_{21}+B_{2} \cos (\theta+\phi)\right)}{s_{1} s_{2}-\left(b_{12}+B_{1} \cos \theta\right)\left(b_{21}+B_{2} \cos (\theta+\phi)\right)} .
\end{aligned}
$$


Substituting these into the third equation of (57), we obtain

$$
\begin{aligned}
& h(\theta) \equiv \mu_{3}+\frac{\left(b_{31}-B_{2} \sin (\theta+\phi)\right)\left[-\mu_{1} s_{2}+\mu_{2}\left(b_{12}+B_{1} \cos \theta\right)\right]}{s_{1} s_{2}-\left(b_{12}+B_{1} \cos \theta\right)\left(b_{21}+B_{2} \cos (\theta+\phi)\right)} \\
& +\frac{\left(b_{32}-B_{1} \sin \theta\right)\left[-\mu_{2} s_{1}+\mu_{1}\left(b_{21}+B_{2}(\cos (\theta+\phi))\right]\right.}{s_{1} s_{2}-\left(b_{12}+B_{1} \cos \theta\right)\left(b_{21}+B_{2} \cos (\theta+\phi)\right)}=0 .
\end{aligned}
$$

Thus the problem of finding nontrivial equilibria of (48) in $\mathcal{H}$ has been reduced to that of finding zeros $\theta_{0}$ of $h(\theta)$ defined by (59). Let us assume that the denominator in (58) and (59) is nonzero. This will hold, for example, if the coefficients $A_{1}, A_{2}$ are sufficiently small compared to the $a_{i j}$. Then $h(\theta)$ is bounded and oscillates with period $2 \pi$ in $\theta$. The graph of $h(\theta)$ shifts up and down as $\mu_{3}$ varies, and as this happens, zeros of $h(\theta)$ will appear and disappear in pairs. The periodicity and continuity of $h(\theta)$ implies that transverse zeros exist in pairs with slopes of opposite sign. Nontransverse zeros with zero slope also are possible but will disappear under perturbation in $\mu_{3}$. Each transverse zero of $h(\theta)$ locally corresponds to a pair of mixed mode phase-locked periodic solutions of the 4-dimensional system, related by the symmetry $\kappa$ as above. The following result is proven in [22].

Lemma 4.1. If the denominator of the function $h(\theta)$ is nonzero, then $h(\theta)$ can have at most four zeros in the interval $[0,2 \pi)$.

The idea of the proof is to rationalize $h(\theta)$ and expand the numerator as a finite Fourier series in $\cos \theta, \sin \theta, \cos 2 \theta$, and $\sin 2 \theta$. It is known that such a sum has at most four zeros, provided that the Fourier coefficients are independent, considered as functions of the normal form parameters. Then the proof is completed by showing that the corresponding Jacobian determinant is generically nonzero [22].

Remark 4.2. The coefficients of the second order terms, $\cos 2 \theta$ and $\sin 2 \theta$, in the finite Fourier series have as a factor the product $A_{1} A_{2}$, while the terms in $\cos \theta$ and $\sin \theta$ have either the factor $A_{1}$ or $A_{2}$. Consequently, if $A_{1} A_{2}$ is sufficiently small, $h(\theta)$ will have at most two zeros.

Since each simple zero of the function $h(\theta)$ in (59) determines one solution for $r_{1}$ and $r_{2}$ in (58), we have the following theorem.

Theorem 4.3. If the denominator of $h(\theta)$ in (59) is nonzero, then corresponding to each transverse zero of $h(\theta)$ there exists a mixed mode phase-locked periodic solution. Locally for generic values of the parameters in the system (48) there are at most four distinct solutions (up to the permutation symmetry $\sigma \sim \kappa$ ).

Figure 1 show examples for the cases when $h(\theta)$ has two, three, or four zeros. Of course, it is possible also for the function $h(\theta)$ to have only one zero (nontransverse) or no zeros. See [22] for more examples and for computation of the mixed mode periodic orbits corresponding to particular zeros of $h(\theta)$.

Corresponding to each equilibrium point $\left(r_{10}, r_{20}, \theta_{0}\right)$ computed as in [22], in the threedimensional state space of (53), there exists a periodic orbit in the 4-dimensional state space of the normal form (54) as follows. Substituting $\left(r_{10}, r_{20}, \theta_{0}\right)$ into the right-hand side of (54) gives $\dot{r}_{1}=0, \dot{r}_{2}=0, \dot{\theta}_{k}=$ constant $_{k}, k=1,2$. But, since $\dot{\theta}_{1}-\dot{\theta}_{2}=\dot{\theta} / 2=0$, these two constants are equal. Let $\hat{\omega}$ denote their common value. (Near the bifurcation point, $\hat{\omega} \approx \omega$.) 


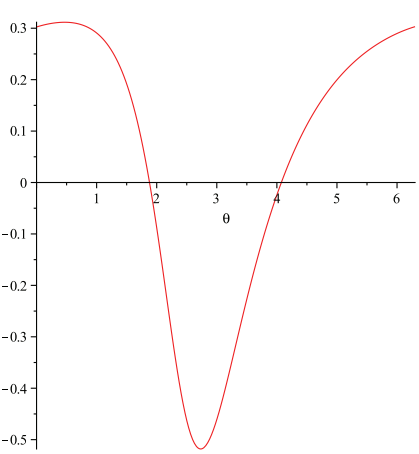

(a)

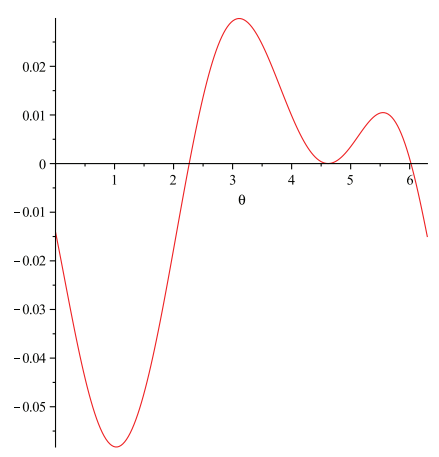

(b)

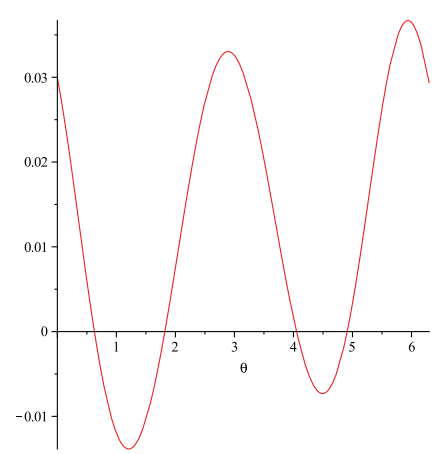

(c)

Figure 1. Zeros of $h(\theta)$. (a) Two zeros. (b) Three zeros (one double). (c) Four zeros.

Then there exists an exact solution of the system (54) given by

$$
r_{1}(t)=r_{10}, \quad r_{2}(t)=r_{20}, \quad \theta_{1}(t)=\hat{\omega} t, \quad \theta_{2}(t)=\hat{\omega} t-\frac{\theta_{0}}{2},
$$

and the corresponding periodic solution in complex coordinates is

$$
z_{1}(t)=r_{10} e^{i \hat{\omega} t}, \quad z_{2}(t)=r_{20} e^{i \hat{\omega} t-\theta_{0} / 2} .
$$

This is a periodic orbit lying on an invariant 2-torus in $\mathbb{R}^{4}$. A second periodic orbit is obtained from this one by application of the $\kappa$-symmetry, as in section 3.4.

5. Quasi-periodic oscillations. In this section, it is assumed that $a_{11}<0$ and $a_{22}<0$, and the truncated normal form is given by (53). This section presents an investigation of (53) in the case where $\dot{\theta}$ does not change sign for all $\theta$ and for $\left(r_{1}, r_{2}\right)$ in some open region $R$ of the positive quadrant. Then the phase-locked mixed mode periodic solutions of the previous section cannot exist in that region. Instead, for appropriate values of the parameters, there exist families of quasi-periodic nonresonant solutions, as described here.

It is convenient in this section to replace the squared terms in (53) with linear ones by the substitution $\rho_{k}=r_{k}^{2}, k=1,2$, and rescaling time by $\tau=2 t$. Since this is a homeomorphism, the resulting two systems are topologically equivalent; in particular, asymptotic stability is preserved. In these new coordinates system (53) becomes

$$
\begin{aligned}
\dot{\rho_{1}} & =\rho_{1}\left[\mu_{1}-\rho_{1}+\left(b_{12}+B_{1} \cos \theta\right) \rho_{2}\right], \\
\dot{\rho_{2}} & =\rho_{2}\left[\mu_{2}+\left(b_{21}+B_{2} \cos (\theta+\phi)\right) \rho_{1}-\rho_{2}\right], \\
\dot{\theta} & =\mu_{3}+\left(b_{31}-B_{2} \sin (\theta+\phi)\right) \rho_{1}+\left(b_{32}-B_{1} \sin \theta\right) \rho_{2} .
\end{aligned}
$$

Here differentiation is with respect to $\tau$.

Consider the case that $\theta$ is a monotone increasing function of $t$, i.e., $\dot{\theta}>0$. (The decreasing case can be transformed to this one by time reversal.) Then $\theta$ may be used as an alias for the time $t$ in $R$, and the 3 -dimensional autonomous system (62) can be transformed to a 2-dimensional nonautonomous system, using

$$
\frac{d \rho_{k}}{d \theta}=\frac{\dot{\rho}_{k}}{\dot{\theta}}, \quad k=1,2,
$$


to obtain

$$
\begin{aligned}
\frac{d \rho_{1}}{d \theta} & =\frac{\rho_{1}\left[\mu_{1}-\rho_{1}+\left(b_{12}+B_{1} \cos \theta\right) \rho_{2}\right]}{\mu_{3}+\left(b_{31}-B_{2} \sin (\theta+\phi)\right) \rho_{1}+\left(b_{32}-B_{1} \sin \theta\right) \rho_{2}}, \\
\frac{d \rho_{2}}{d \theta} & =\frac{\rho_{2}\left[\mu_{2}+\left(b_{21}+B_{2} \cos (\theta+\phi)\right) \rho_{1}-\rho_{2}\right]}{\mu_{3}+\left(b_{31}-B_{2} \sin (\theta+\phi)\right) \rho_{1}+\left(b_{32}-B_{1} \sin \theta\right) \rho_{2}} .
\end{aligned}
$$

This is a nonlinear nonautonomous 2-dimensional system with $2 \pi$-periodic parametric forcing. There is an existing body of tools for such systems [1, 24].

5.1. Existence theory. The assumption $\dot{\theta}>0$ places restrictions on some of the parameter values. For example, if $X$ is the positive quadrant, the assumption can be satisfied for all $\phi$, all $\theta$, and all $\left(\rho_{1}, \rho_{2}\right) \in X$ if

$$
B_{1}<b_{32}, \quad B_{2}<b_{31}, \quad \text { and } \mu_{3}>0 .
$$

Recall that the constants $B_{1}$ and $B_{2}$ are necessarily nonnegative. Here it is assumed also that $B_{1}$ and $B_{2}$ are nonzero; the case where both are zero is discussed in section 3.6.

The following classical theorem plays a central role in proving existence of periodic solutions of a differential equation of the form (63). Let $E$ be a finite-dimensional real Hilbert space, and let $\langle\cdot \mid \cdot\rangle$ denote the inner product in $E$. Assume that $f \in C^{0,1-}(\mathbb{R} \times E, E)$ and $f$ is $T$-periodic with respect to $t \in \mathbb{R}$ for some $T>0$. The notation $f \in C^{0,1-}$ means that $f$ is continuous in $t \in \mathbb{R}$ and Lipschitz continuous in $x \in E$.

Theorem 5.1 (Krasnoselskii [24]). Let $E$ and $f$ be as in the paragraph above. Moreover, let $V \in C^{1}(E, \mathbb{R})$ be such that, for some $r_{0}>0$, we have

$$
\langle\nabla V(x) \mid f(t, x)\rangle<0 \quad \forall|x| \geq r_{0}, 0 \leq t \leq T .
$$

If either $V(x) \rightarrow \infty$ or $V(x) \rightarrow-\infty$ as $|x| \rightarrow \infty$, then the differential equation

$$
\dot{x}=f(t, x)
$$

has at least one T-periodic solution.

Proof. A proof based on topological degree arguments is presented in [1, 24].

Since $\rho_{1}$ and $\rho_{2}$ are nonnegative in (63), the state space of (63) is not a Hilbert space $E$. In order to use Krasnoselskii's theorem, we must modify it so that, instead of requiring $x \in E$, it is sufficient that $x \in X \subset E$, where $X$ is a suitable open set, in our case the positive quadrant in $E=\mathbb{R}^{2}$. A proof of this modified theorem is given in [22], using topological degree theory, based on the proof of Krasnoselskii's theorem presented in [1].

Theorem 5.2 (Kitanov [22]). Let $X$ be an open set in a finite-dimensional real Hilbert space $E$, and assume that $f \in C^{0,1-}(\mathbb{R} \times X, E)$ is T-periodic in $t \in \mathbb{R}$. Suppose that there is an open set $U$ with $\bar{U} \subset X$ and $a V \in C^{1}(X, \mathbb{R})$ such that

$$
\langle\nabla V(x) \mid f(t, x)\rangle<0 \quad \forall x \in X \backslash U, t \in[0, T] .
$$

If either $V(x) \rightarrow \infty$ or $V(x) \rightarrow-\infty$ as $x \rightarrow \partial X$, then the differential equation

$$
\dot{x}=f(t, x)
$$


has at least one T-periodic solution.

Proof. See Kitanov [22].

In order to apply Theorem 5.2 to (63), an appropriate Lyapunov-like function $V\left(\rho_{1}, \rho_{2}\right)$ is constructed that goes to infinity when $\left(\rho_{1}, \rho_{2}\right)$ approaches the boundary of the positive quadrant. $V$ is chosen as a positive definite function with respect to the nontrivial equilibrium of (63) that exists in the special case $B_{1}=B_{2}=0$. Under suitable values for the other parameters it is shown that this equilibrium is in the positive quadrant and is stable. Then, allowing $B_{1}$ and $B_{2}$ to be nonzero but not too large, it is shown that the value of $V$ continues to decrease along trajectories of (63) except in some small region $U$ near the equilibrium.

Theorem 5.3. Choose any positive number $\epsilon<2$. If (64) holds and

$$
\left|b_{12}+b_{21}\right|<2-\epsilon \quad \text { and } \quad B_{1}, B_{2}<\frac{\epsilon}{5},
$$

then there exists an open region in $\left(\mu_{1}, \mu_{2}\right)$-space bounded by two rays extending from the origin such that for any values of $\left(\mu_{1}, \mu_{2}\right)$ in this region and any value of $\phi$ system (63) has at least one $2 \pi$-periodic solution.

Proof. Let $X$ be the positive quadrant $\left\{\rho_{1}>0, \rho_{2}>0\right\}$, and let $f$ be the vector field of (63), namely

$$
\begin{aligned}
f_{1} & =\frac{\rho_{1}\left[\mu_{1}-\rho_{1}+\left(b_{12}+B_{1} \cos \theta\right) \rho_{2}\right]}{\mu_{3}+\left(b_{31}-B_{2} \sin (\theta+\phi)\right) \rho_{1}+\left(b_{32}-B_{1} \sin \theta\right) \rho_{2}}, \\
f_{2} & =\frac{\rho_{2}\left[\mu_{2}+\left(b_{21}+B_{2} \cos (\theta+\phi)\right) \rho_{1}-\rho_{2}\right]}{\mu_{3}+\left(b_{31}-B_{2} \sin (\theta+\phi)\right) \rho_{1}+\left(b_{32}-B_{1} \sin \theta\right) \rho_{2}} .
\end{aligned}
$$

Note that the denominator of both $f_{1}$ and $f_{2}$ is $\dot{\theta}$. Since (64) holds we know that $\dot{\theta}$ is positive for all $\left(\theta, \rho_{1}, \rho_{2}\right) \in(\mathbb{R}, X)$, and hence $f$ is in $C^{0,1-}\left(\mathbb{R} \times X, \mathbb{R}^{2}\right)$.

In the special case $B_{1}=B_{2}=0$, the nontrivial equilibrium of system (63) satisfies

$$
\begin{aligned}
& \mu_{1}-\rho_{1}+b_{12} \rho_{2}=0, \\
& \mu_{2}+b_{21} \rho_{1}-\rho_{2}=0 .
\end{aligned}
$$

The solution of the above system is

$$
q_{1}:=\rho_{1}^{*}=\frac{\mu_{1}+\mu_{2} b_{12}}{1-b_{12} b_{21}}, \quad q_{2}:=\rho_{2}^{*}=\frac{\mu_{2}+\mu_{1} b_{21}}{1-b_{12} b_{21}} .
$$

Condition (67) implies that $b_{12} b_{21}<1$; thus the denominators in the above expressions are positive. Further, (70) defines an invertible linear transformation from the $\left(\mu_{1}, \mu_{2}\right)$-plane to the $\left(q_{1}, q_{2}\right)$-plane since the determinant of the transformation matrix is $1 /\left(1-b_{12} b_{21}\right)>0$, and therefore distinct rays from the origin in the $\left(\mu_{1}, \mu_{2}\right)$-plane map to distinct rays from the origin in the $\left(q_{1}, q_{2}\right)$-plane and vice versa. The requirement that the nontrivial equilibrium lies in the first quadrant, $q_{1}>0, q_{2}>0$, therefore transforms to a requirement that $\left(\mu_{1}, \mu_{2}\right)$ lies in some sector bordered by two rays coming from the origin.

Define

$$
V\left(\rho_{1}, \rho_{2}\right)=\rho_{1}-q_{1}-q_{1} \ln \left(\frac{\rho_{1}}{q_{1}}\right)+\rho_{2}-q_{2}-q_{2} \ln \left(\frac{\rho_{2}}{q_{2}}\right)
$$


then $V \rightarrow \infty$ as $\left(\rho_{1}, \rho_{2}\right) \rightarrow \partial X, V$ is positive definite in $X$ with respect to $\left(q_{1}, q_{2}\right)$, and

$$
\nabla V=\left(1-\frac{q_{1}}{\rho_{1}}, 1-\frac{q_{2}}{\rho_{2}}\right)=\left(\frac{\rho_{1}-q_{1}}{\rho_{1}}, \frac{\rho_{2}-q_{2}}{\rho_{2}}\right) .
$$

Therefore,

$$
\langle\nabla V \mid f\rangle=\frac{\left(\rho_{1}-q_{1}\right)\left(\mu_{1}-\rho_{1}+b_{12} \rho_{2}+B_{1} \cos \theta \rho_{2}\right)+\left(\rho_{2}-q_{2}\right)\left(\mu_{2}+b_{21} \rho_{1}+B_{2} \cos (\theta+\phi) \rho_{1}-\rho_{2}\right)}{\dot{\theta}} .
$$

Rearranging (70) gives $\mu_{1}=q_{1}-b_{12} q_{2}$ and $\mu_{2}=q_{2}-b_{21} q_{1}$, so

$$
\begin{aligned}
\dot{\theta}\langle\nabla V \mid f\rangle=- & \left(\rho_{1}-q_{1}\right)^{2}-\left(\rho_{2}-q_{2}\right)^{2}+\left[b_{12}+b_{21}+B_{1} \cos \theta+B_{2} \cos (\theta+\phi)\right]\left(\rho_{1}-q_{1}\right)\left(\rho_{2}-q_{2}\right) \\
& +B_{1} q_{2}\left(\rho_{1}-q_{1}\right) \cos \theta+B_{2} q_{1}\left(\rho_{2}-q_{2}\right) \cos (\theta+\phi) .
\end{aligned}
$$

Since $\dot{\theta}>0$ the sign of $\langle\nabla V \mid f\rangle$ changes when $\left(X_{1}, X_{2}\right):=\left(\rho_{1}-q_{1}, \rho_{2}-q_{2}\right)$ crosses the curve

$$
-X_{1}^{2}-X_{2}^{2}+\beta X_{1} X_{2}+B_{1} q_{2} X_{1} \cos \theta+B_{2} q_{1} X_{2} \cos (\theta+\phi)=0,
$$

where $\beta=b_{12}+b_{21}+B_{1} \cos \theta+B_{2} \cos (\theta+\phi)$. Using (67),

$$
|\beta| \leq\left|b_{12}+b_{21}\right|+B_{1}+B_{2}<2-\epsilon+\frac{2 \epsilon}{5}=2-\frac{3 \epsilon}{5},
$$

and therefore the discriminant $\Delta$ of the quadratic polynomial in $\left(X_{1}, X_{2}\right)$ given by the lefthand side of (72) satisfies

$$
\Delta:=\beta^{2}-4(-1)(-1)<4-\frac{12 \epsilon}{5}+\frac{9 \epsilon^{2}}{25}-4=\frac{3 \epsilon}{5}\left(\frac{3 \epsilon}{5}-4\right)<0,
$$

since $\epsilon<2$. Thus for each fixed $\theta$ and $\phi$, (72) represents an ellipse. The point $\left(X_{1}, X_{2}\right)=(0,0)$ satisfies (72); hence the ellipse is not imaginary. Further, since the coefficients in front of the homogeneous quadratic terms in (72) are negative, and since $\dot{\theta}$ is positive, $\langle\nabla V \mid f\rangle$ will be negative outside this ellipse. In the special case $B_{1}=B_{2}=0$, this ellipse degenerates to the single point $\left(X_{1}, X_{2}\right)=(0,0)$, that is, $\left(\rho_{1}, \rho_{2}\right)=\left(q_{1}, q_{2}\right)$, and therefore $V$ is a Lyapunov function displaying global asymptotic stability of the equilibrium in this case.

To show that the ellipse (72) lies in the positive quadrant of the $\left(\rho_{1}, \rho_{2}\right)$-plane, that is, $X_{1}>-q_{1}$ and $X_{2}>-q_{2}$, it is sufficient to show that the extreme points of the ellipse do so. The normal to the ellipse is given by

$$
\vec{n}=\left[\begin{array}{c}
-2 X_{1}+\beta X_{2}+B_{1} q_{2} \cos \theta \\
-2 X_{2}+\beta X_{1}+B_{2} q_{1} \cos (\theta+\phi)
\end{array}\right] .
$$

The extreme top and bottom points of the ellipse are located where the first component of this normal is zero, and the extreme left and right points where the second component is zero. Setting the first component to zero, solving for $X_{1}$, substituting this back into (72), and simplifying gives

$$
P\left(X_{2}\right):=\Delta X_{2}^{2}+\left[2 \beta B_{1} q_{2} \cos \theta+4 B_{2} q_{1} \cos (\theta+\phi)\right] X_{2}+B_{1}^{2} q_{2}^{2} \cos ^{2} \theta=0 .
$$


$P$ is a parabola in $X_{2}$ opening downward since, as shown in (74), $\Delta<0$, and further, $P(0) \geq 0$. To show that both roots of (75) lie to the right of $-q_{2}$ it is therefore sufficient to show that $P\left(-q_{2}\right)<0$ :

$$
\begin{aligned}
P\left(-q_{2}\right) & =\Delta q_{2}^{2}-\left[2 \beta B_{1} q_{2} \cos \theta+4 B_{2} q_{1} \cos (\theta+\phi)\right] q_{2}+B_{1}^{2} q_{2}^{2} \cos ^{2} \theta \\
& =q_{2}^{2}\left[\Delta-2 \beta B_{1} \cos \theta-4 B_{2} \frac{q_{1}}{q_{2}} \cos (\theta+\phi)+B_{1}^{2} \cos ^{2} \theta\right] .
\end{aligned}
$$

Using the theorem conditions (67), along with (73) and (74), this becomes

$$
P\left(-q_{2}\right)<q_{2}^{2}\left[\frac{3 \epsilon}{5}\left(\frac{3 \epsilon}{5}-4\right)+2\left(2-\frac{3 \epsilon}{5}\right) \frac{\epsilon}{5}+4 \frac{\epsilon}{5} \frac{q_{1}}{q_{2}}+\frac{\epsilon^{2}}{25}\right]
$$

which simplifies to

$$
P\left(-q_{2}\right)<q_{2}^{2}\left(\frac{4 \epsilon}{5}\right)\left[\frac{q_{1}}{q_{2}}-2+\frac{\epsilon}{5}\right] .
$$

Since $0<\epsilon<2, P\left(-q_{2}\right)<0$ can be satisfied if

$$
\frac{q_{1}}{q_{2}}<\frac{8}{5}
$$

Thus if (76) holds, the ellipse (72) will lie above the line $X_{2}=-q_{2}$; that is, $\rho_{2}>0$. In a completely analogous way, starting by setting the second component of $\vec{n}$ to zero, the ellipse can be shown to lie to the right of the line $X_{1}=-q_{1}$; that is, $\rho_{1}>0$, provided that

$$
\frac{q_{2}}{q_{1}}<\frac{8}{5}
$$

Clearly (76), (77) and the positivity conditions $q_{1}>0, q_{2}>0$ are simultaneously satisfied in the region of the first quadrant bounded below by the ray $q_{2}=5 q_{1} / 8$ and above by the ray $q_{2}=8 q_{1} / 5$. As remarked earlier, this region corresponds to an open wedge-shaped region $W$ in the $\left(\mu_{1}, \mu_{2}\right)$-plane.

Equation (72) represents an ellipse for each $\theta$ and each $\phi$. Let $\hat{U}$ be the union of all these ellipses and their interiors, that is,

$$
\hat{U}=\bigcup_{\substack{0 \leq \theta \leq 2 \pi \\ 0 \leq \phi \leq 2 \pi}}\left\{\left(X_{1}, X_{2}\right) \mid-X_{1}^{2}-X_{2}^{2}+\beta X_{1} X_{2}+B_{1} q_{2} X_{1} \cos \theta+B_{2} q_{1} X_{2} \cos (\theta+\phi) \geq 0\right\} .
$$

Since the ellipse is continuous in both $\theta$ and $\phi$, and since the extreme points of each ellipse lie above $X_{2}=-q_{2}$ and to the right of $X_{1}=-q_{1}$, it follows that the union of the ellipses over all $\theta$ and $\phi$ in the compact set $[0,2 \pi] \times[0,2 \pi]$ is a closed set that lies strictly above $X_{2}=-q_{2}$ and to the right of $X_{1}=-q_{1}$. Let $U$ be any open set above $X_{2}=-q_{2}$, to the right of $X_{1}=-q_{1}$, and enclosing $\hat{U}$. Then under the hypotheses of the theorem, for $\left(\mu_{1}, \mu_{2}\right) \in W$ and for any $\phi$, the function $V$ satisfies the conditions of Theorem 5.2 for the system (63). Therefore (63) has at least one $2 \pi$-periodic solution. 


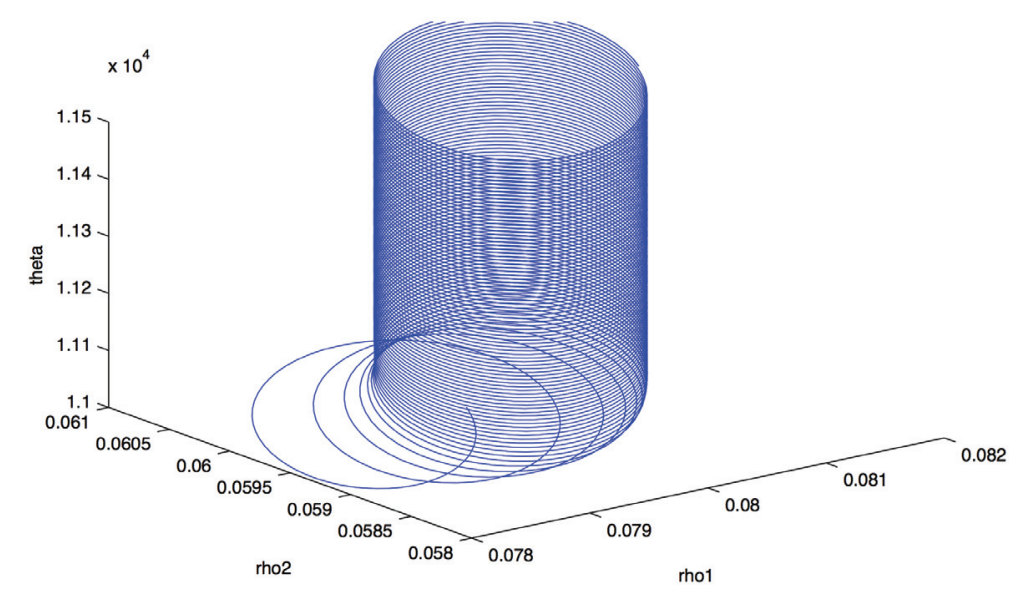

Figure 2. Approach to a periodic solution in $\left(\rho_{1}, \rho_{2}, \theta\right)$ coordinates.

Remark 5.4. As noted by Krasnoselskii [24], an a priori bound on the periodic solution is provided by $V$. In the case of the function $V$ used in the proof of Theorem 5.3, the periodic solution of (63) must reside in the set

$$
\left\{\left(\rho_{1}, \rho_{2}\right) \mid V\left(\rho_{1}, \rho_{2}\right) \leq \max _{\left(r_{1}, r_{2}\right) \in \partial U} V\left(r_{1}, r_{2}\right)\right\} .
$$

Remark 5.5. The existence of periodic solutions of (63) implies the existence of aperiodic solutions and more complex dynamics for the 4-dimensional normal form equations (36) or (38).

5.2. Phase portraits for quasi-periodic orbits. This section presents phase portraits of representative orbits that exist for the 4-dimensional normal form equations, under the hypotheses of Theorem 5.3. Under those assumptions, the trivial equilibrium with $B_{1}=B_{2}=0$ has negative trace and positive determinant, so it is asymptotically stable. This guarantees (locally) that the periodic orbit is orbitally asymptotically stable also. Therefore, in order to compute this orbit, it is sufficient to choose a nearby initial condition and then integrate the system until the transients die out and the solution converges to the desired orbit. Proceeding in this fashion with the 2-dimensional system (63), numerical integration converges to a periodic orbit in the $\rho_{1}>0, \rho_{2}>0$ quadrant; see [22].

Next consider the 3-dimensional truncated system (53). Numerical integration of this system with parameter values satisfying Theorem 5.3 yields the same periodic orbit as for (63), but now in a 3-dimensional "slab": $r_{1}>0, r_{2}>0$, and $0 \leq \theta \leq 2 \pi$. Period $2 \pi$ in $\theta$ means that the orbit leaves through the top of the slab at the same $\left(r_{1}, r_{2}\right)$ as the point where it enters through the bottom of the slab. See Figure 2 for numerical solution of (53), which converges to such a periodic orbit.

In order to see that a periodic orbit of (53) is not in general periodic for the full system, we consider the 4-dimensional normal form in polar coordinates (54). It is clear that the right-hand side of this system depends on only three variables, $\left(r_{1}, r_{2}, \theta\right)$, and not on the individual $\theta_{1}, \theta_{2}$. For initial conditions we can assume $\theta(0)=0$; that means $\theta_{1}(0)=\theta_{2}(0)$, 


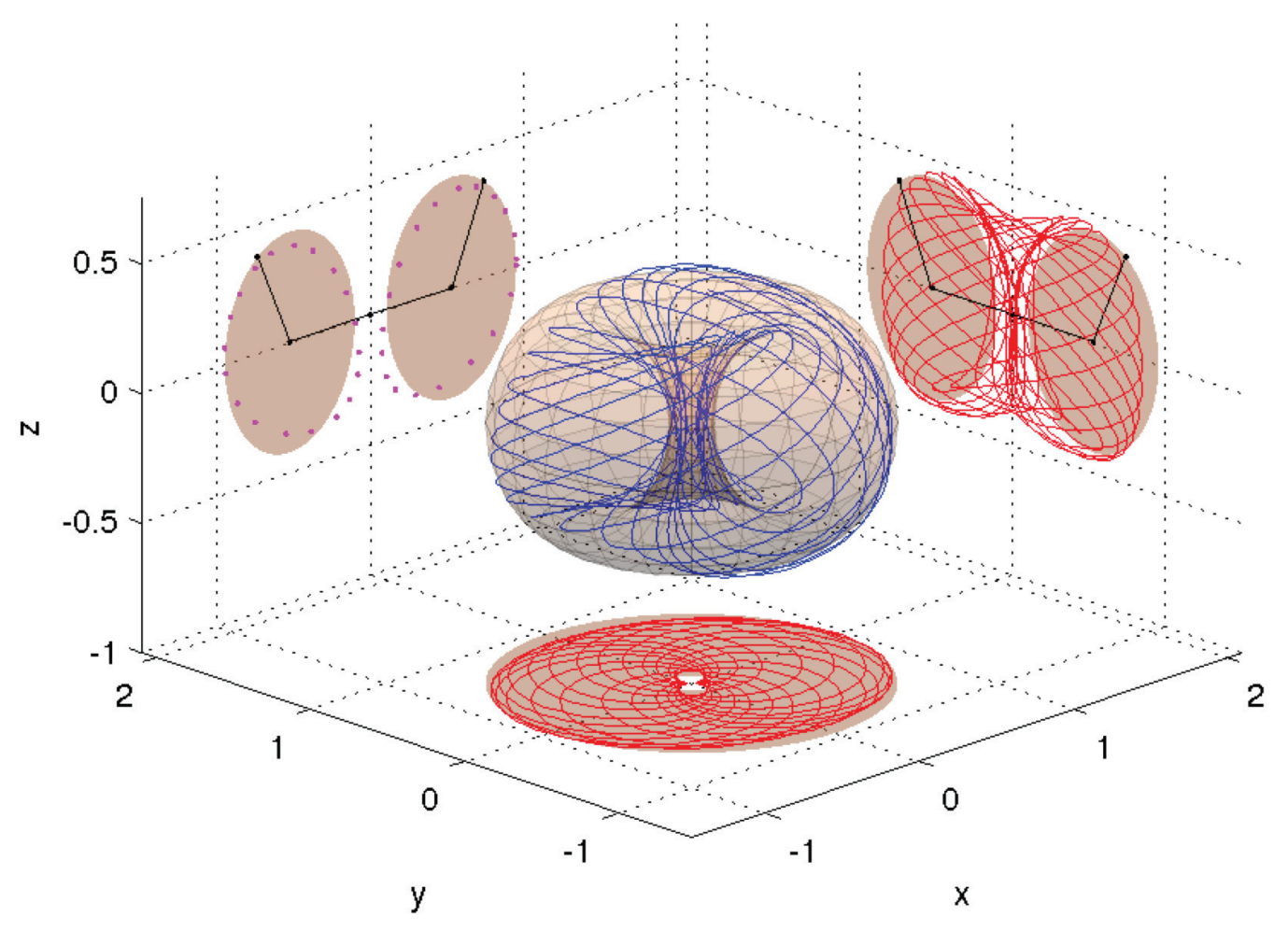

Figure 3. Toroidal breather. Computed quasi-periodic solution in three dimensions (blue) with two planar projections on coordinate planes (red) and Poincaré map for $y=0$ (magenta). The breathing torus and its cross sections at $x=0, y=0$, and $z=0$ (brown) are shown as they appear for the final point of the trajectory. This is the final frame from a computed video of the toroidal breather (83946_01.avi [local/web 164MB]). At the beginning of the video, the relationship between the polar coordinates $\left(r_{1}, r_{2}, \theta_{1}, \theta_{2}\right)$ of the normal form and the Cartesian coordinates $(x, y, z)$ of the plot is illustrated. The 2 -torus at $t=0$ is drawn, and its cross sections at $x=0, y=0$, and $z=0$ are projected onto the background planes. The distances $r_{1}$ and $r_{2}$ are drawn as black rods on the $x=0$ and $y=0$ projections. Time then progresses from $t=0$, and the leading end of the trajectory is drawn in blue. The trailing end of the trajectory disappears in order to keep the central part of the figure uncluttered. As time increases, the 2-torus (and its cross sections) are slowly changing since both $r_{1}$ and $r_{2}$ vary slowly with time. The leading end of the trajectory remains on the surface of the "breathing" 2-torus. At some points in the video it can be seen that before the trailing end of the trajectory disappears, it is off the instantaneous 2-torus. The projections of the entire trajectory are shown in red overlaid on the $x=0$ and $z=0$ cross sections. The Poincaré map of the trajectory as it crosses $y=0$ is plotted as magenta dots overlaid on the $y=0$ cross section. The sequence continues for four periods in $\theta$. At the end of the sequence, the entire trajectory is shown in blue.

and without loss of generality this common initial value can be zero. Then $r_{1}(0)$ and $r_{2}(0)$ will be the values where the periodic orbit enters the slab at $\theta=0$ in the previous calculation. Numerical integration of (54) gives a data file representing an orbit in a 4-dimensional space: $\left(r_{1}(t), r_{2}(t), \theta_{1}(t), \theta_{2}(t)\right)$. Here $\theta_{1}(t)$ and $\theta_{2}(t)$ vary much faster than $\theta(t)$, because they have angular frequency near $\omega$ which is order one, whereas $\theta(t)$ has angular frequency that is small compared to one. Thus a data file with $\theta$ between 0 and $2 \pi$ represents many oscillations in the $\theta_{1}$ and $\theta_{2}$ coordinates. This computed orbit is shown in Figure 3. The parameter values 
are $\mu_{1}=0.28, \mu_{2}=0.09, \phi=2.0, \omega=2.0, b_{12}=-0.25, b_{21}=0.3, c_{11}=0.5, c_{12}=0.7, c_{21}=$ $0.1, c_{22}=0.2, B_{1}=0.35, B_{2}=0.38, \gamma_{0}=0.15$, and $\eta_{0}=0.05$.

This orbit is plotted as follows. Suppose $r_{1}$ is greater than $r_{2}$; otherwise interchange $r_{1}$ and $r_{2}$. First, plot $\left(r_{1}(t), \theta_{1}(t)\right)$ as usual in a plane with polar coordinates. This will not be a periodic orbit in general, because it is a projection of something more complicated in four dimensions. Now at each point of this orbit construct a transverse 2-dimensional Poincaré section in 3-dimensional space. In this 2-dimensional Poincaré section, plot the $\left(r_{2}(t), \theta_{2}(t)\right)$ coordinates, with origin at the point where the previous orbit intersects the Poincaré section, and let this Poincaré section swing around the $\left(r_{1}(t), \theta_{1}(t)\right)$ orbit as $t$ increases. The resulting compound orbit resembles an orbit on an invariant 2-torus (see Figure 3), where the coordinates are

$$
x=\left(r_{1}+r_{2} \cos \theta_{2}\right) \cos \theta_{1}, \quad y=\left(r_{1}+r_{2} \cos \theta_{2}\right) \sin \theta_{1}, \quad z=r_{2} \sin \theta_{2} .
$$

In addition, however, $r_{1}$ and $r_{2}$, which are the two principal radii of the torus, vary periodically in $\theta$. In Figure $3, r_{1}$ varies between 0.464 and 0.535 , while $r_{2}$ varies between 0.347 and 0.456 . This means that the invariant set in the previous paragraph that "resembles" a 2torus is actually an invariant 3 -torus in the 4-dimensional state space, with three independent frequencies and with normal hyperbolicity inherited from the periodic orbit of Theorem 5.3. Since $\dot{\theta}_{1}=\dot{\theta}_{2}+\dot{\theta} / 2$ and $|\dot{\theta}| \ll\left|\dot{\theta}_{1}\right|$, the rotation numbers of this orbit (time-averages of the ratios of the angular frequencies) are either irrational or rational but a ratio involving very large integers. Therefore, the orbit is either quasi-periodic or periodic with a very long period; the latter case in practice, both experimentally and computationally, may be indistinguishable from quasi-periodic. Loosely, we refer to the orbit as quasi-periodic in either case, without actually computing the rotation number.

In the 3-dimensional representation used for Figure 3, the third frequency implied by Theorem 5.3 is manifested as a slow periodic modulation of the 2-torus; that is, the two principal radii of the 2-torus slowly vary periodically in time, with small amplitude. In a 3dimensional projection such as Figure 3, the cumulative effect over a long time is a thickening of the 2-torus manifold into a toroidal shell. This type of 3-dimensional attractor may be called a toroidal breather.

6. Secondary bifurcations. In section 4.1 it was shown that the system displays two primary Hopf bifurcations from the trivial solution, each giving rise to a periodic solution in one of the two normal modes, that is, either in-phase, with $r_{1}>0, r_{2}=0$, or anti-phase, with $r_{1}=0, r_{2}>0$. In this section we explore secondary bifurcations from these normal mode periodic solutions. The secondary bifurcations of codimension one give birth either to mixed mode phase-locked periodic solutions as studied in section 4 or to quasi-periodic solutions of "breather" type as in section 5. We show that an Arnold tongue plays a fundamental role in the determination of which of these two types of solution arises in this secondary bifurcation. A detailed numerical study in section 6.3 extends these analytical results, using MATLAB. In addition, there exist at least three types of secondary bifurcations of codimension two, explored analytically in subsection 6.1.3 and numerically in section 6.3. For most of this section, as in section 5, we assume $a_{11}<0, a_{22}<0$, so that both of the primary Hopf bifurcations are supercritical and stable. The subcritical case is considered briefly in subsection 6.3.4. 
6.1. Secondary bifurcations from the in-phase normal mode. In this subsection, the case of secondary bifurcation from the in-phase normal mode solution, with $r_{1}=\sqrt{\mu_{1}}>0$ and $r_{2}=0$, is considered. The anti-phase case is similar, as shown in subsection 6.2.

The truncated 3-dimensional system has the form (53), which we repeat here for convenience as

$$
\begin{aligned}
\dot{r}_{1} & =r_{1}\left[\mu_{1}-r_{1}^{2}+\left(b_{12}+B_{1} \cos \theta\right) r_{2}^{2}\right], \\
\dot{r}_{2} & =r_{2}\left[\mu_{2}+\left(b_{21}+B_{2} \cos (\theta+\phi)\right) r_{1}^{2}-r_{2}^{2}\right], \\
\dot{\theta} & =2\left[\mu_{3}+\left(b_{31}-B_{2} \sin (\theta+\phi)\right) r_{1}^{2}+\left(b_{32}-B_{1} \sin \theta\right) r_{2}^{2}\right] .
\end{aligned}
$$

System (78) is well-posed and uniquely defines solutions $\left(r_{1}(t), r_{2}(t), \theta(t)\right)$ for any given initial point $\left(r_{1}(0), r_{2}(0), \theta(0)\right)$. At any point on the in-phase normal mode branch of solutions $\left(r_{1}, r_{2}\right)=\left(\sqrt{\mu_{1}}, 0\right)$, system $(78)$ reduces to a single equation for $\theta$, given by

$$
\dot{\theta}=2\left(\mu_{3}+b_{31} \mu_{1}\right)-2 B_{2} \mu_{1} \sin (\theta+\phi)
$$

and this too is well-posed. However, a problem arises in translating these solutions $\left(\sqrt{\mu_{1}}, 0, \theta\right)$ back to the 4-dimensional system, for example expressed in coordinates $\left(r_{1}, \theta_{1}, r_{2}, \theta_{2}\right)$; see (54). By definition, $\theta \equiv \theta_{1}-\theta_{2}$, but since $\theta_{2}$ is undefined when $r_{2}=0$, this means the value of $\theta$ is arbitrary on the in-phase normal mode branch. In particular, any bifurcation that occurs in the $\theta$ variable while $r_{2}=0$ is a "phantom bifurcation" for the original system, since it is not visible there. One might think of this bifurcation as occurring inside the point in polar coordinates $\left(r_{2}=0, \theta=\right.$ arbitrary). Nevertheless, for arbitrarily small $r_{2}>0, \theta_{2}$ is well defined, as is $\theta \equiv \theta_{1}-\theta_{2}$. Let us return to this issue after further investigation of (78).

In the next two subsections, we consider two mutually exclusive cases for system (78):

1. System (78) has an equilibrium point $\left(r_{1}, r_{2}, \theta\right)=\left(\sqrt{\mu_{1}}, 0, \theta_{0}\right)$ for some fixed $\theta_{0}$. The possibility of multiple equilibrium points $\theta_{0}$ for the same $\left(r_{1}, r_{2}\right)$ is allowed. This case is shown in subsection 6.1.1 to correspond to the interior of an Arnold tongue and to give rise to periodic solutions.

2. There is no equilibrium for the $\dot{\theta}$ equation in (78) when $\left(r_{1}, r_{2}\right)=\left(\sqrt{\mu_{1}}, 0\right)$, that is, for (79). Then, on the in-phase normal mode solution, $\dot{\theta}$ is of one sign, either always positive or always negative, but it is periodic, continuous, and bounded away from zero. In subsection 6.1.2, this case is shown to correspond to the exterior of an Arnold tongue and to give rise to quasi-periodic solutions.

6.1.1. Periodic solutions and Arnold tongues. Assume that system (78) has an equilibrium point $\left(r_{1}, r_{2}, \theta\right)=\left(\sqrt{\mu_{1}}, 0, \theta_{0}\right)$, and begin the analysis of secondary bifurcations with the Jacobian matrix of system (78), evaluated at this equilibrium, namely,

$$
J_{1}=\left[\begin{array}{ccc}
-2 \mu_{1} & 0 & 0 \\
0 & \mu_{2}+\left(b_{21}+B_{2} \cos \left(\theta_{0}+\phi\right)\right) \mu_{1} & 0 \\
4 \sqrt{\mu_{1}}\left(b_{31}-B_{2} \sin \left(\theta_{0}+\phi\right)\right) & 0 & -2 B_{2} \mu_{1} \cos \left(\theta_{0}+\phi\right)
\end{array}\right] .
$$

Let us denote the diagonal elements of $J_{1}$ by $\left\{\lambda_{1}, \lambda_{2}, \lambda_{3}\right\}$, respectively. Clearly these are the eigenvalues of $J_{1}$. They can be used to determine stability and bifurcations. A zero eigenvalue of $J_{1}$ in general corresponds to a secondary bifurcation. Note that $\lambda_{1} \equiv-2 \mu_{1}<0$ on the 
(a)

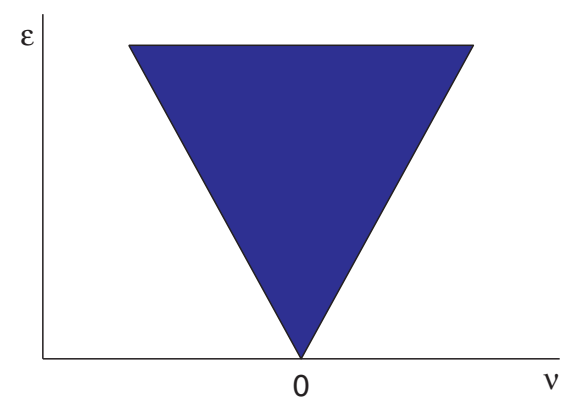

(b)

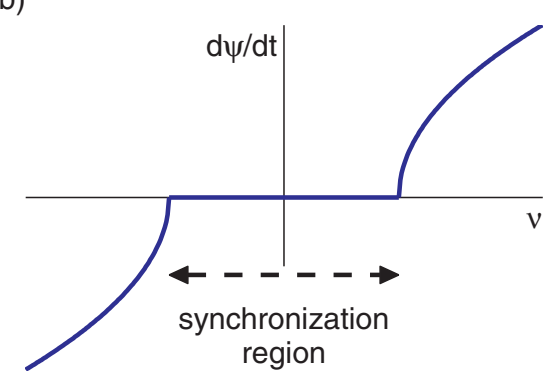

Figure 4. (a) Arnold tongue for the phase equation (84). At points $(\nu, \epsilon)$ lying inside the triangular "tongue" region $|\nu|<\epsilon$, (84) has a pair of equilibrium solutions, satisfying $\psi=\sin ^{-1}\left(\frac{\nu}{\epsilon}\right)$. (b) The dependence on $\nu$ of the time-average of $\dot{\psi}$ is shown, along a slice through the tongue for fixed $\epsilon>0$. Inside the tongue, the mean of the phase frequency $\dot{\psi}$ goes to 0 asymptotically in $t$ as the two normal modes become synchronized. Outside but near the tongue, the mean phase frequency can change rapidly.

in-phase normal mode branch, implying asymptotic stability and no bifurcation in the $r_{1}$ direction. Typically, a secondary bifurcation that gives rise to a solution with $r_{2}>0$ will occur when

$$
\lambda_{2}\left(\theta_{0}\right) \equiv \mu_{2}+b_{21} \mu_{1}+B_{2} \mu_{1} \cos \left(\theta_{0}+\phi\right)=0 .
$$

Here $\theta_{0}$ is the assumed equilibrium point of the $\dot{\theta}$ phase equation in (78), which simplifies at a point on the in-phase primary branch to (79). Because $r_{2}$ is an amplitude coordinate, the secondary bifurcation corresponding to condition (81) is of pitchfork type. Because we have assumed $a_{22}<0$ in (78), the new solution (with $r_{2}>0$ ) bifurcates supercritically in $\mu_{2}$.

The possibility of vanishing of the third eigenvalue, $\lambda_{3} \equiv-2 B_{2} \mu_{1} \cos \left(\theta_{0}+\phi\right)$ in (80), is investigated below in this subsection, where it is seen that $\lambda_{3}=0$ corresponds to the boundaries of an Arnold tongue. The simultaneous vanishing of both eigenvalues $\lambda_{2}=\lambda_{3}=0$ (a codimension-two double zero secondary bifurcation) is explored in subsection 6.1.3.

Define new parameters to simplify (79) and (81) by the nonsingular transformation

$$
\left[\begin{array}{l}
\epsilon \\
\delta \\
\nu
\end{array}\right]=\left[\begin{array}{lll}
B_{2} & 0 & 0 \\
b_{21} & 1 & 0 \\
b_{31} & 0 & 1
\end{array}\right]\left[\begin{array}{l}
\mu_{1} \\
\mu_{2} \\
\mu_{3}
\end{array}\right], \quad \psi=\theta+\phi, \quad \psi_{0}=\theta_{0}+\phi, \quad \tau=2 t .
$$

Then (81) and (79) respectively take the simpler form

$$
\begin{aligned}
0 & =\delta+\epsilon \cos \left(\psi_{0}\right), \\
\dot{\psi} & =\nu-\epsilon \sin (\psi),
\end{aligned}
$$

where $\dot{\psi}=\frac{d \psi}{d \tau}$. The phase differential equation (84) is sometimes called the Adler equation; see [31]. This equation exhibits the well-known phenomenon of Arnold tongues, shown in Figure 4(a). The Arnold tongue for the Adler equation is defined as the set in the $(\nu, \epsilon)$ plane for which (84) has an equilibrium solution $\psi_{0}$, i.e., $\dot{\psi}=0$. This is possible if and 
only if $|\nu / \epsilon| \leq 1$, and therefore the boundaries of the Arnold tongue are the lines $\nu= \pm \epsilon$. Only $\epsilon>0$ is allowed, because $B_{2}>0$ by definition and $\mu_{1}>0$ by hypothesis. At each point interior to the tongue $|\nu|<\epsilon$, the Adler equation (84) has two distinct equilibria $\psi_{0}=\psi_{j} \in\left(-\frac{\pi}{2}, \frac{3 \pi}{2}\right), j=1,2$, defined by

$$
\psi_{1}=\arcsin \left[\frac{\nu}{\epsilon}\right], \quad \psi_{2}=\pi-\psi_{1},
$$

where $\psi_{1}$ is the principal value of $\arcsin$ in $(-\pi / 2, \pi / 2)$. The equilibrium $\psi_{1}$ is asymptotically stable, and $\psi_{2} \in(\pi / 2,3 \pi / 2)$ is unstable, as solutions of the phase equation (84). These two equilibria inside the tongue merge and disappear on outward crossing of the boundary of the tongue $|\nu|=\epsilon$, in what is called a saddle-node homoclinic bifurcation on an invariant circle $\left[25\right.$, p. 250]. On the boundary $\nu=\epsilon$, the two equilibria merge at the value $\psi_{1}=\psi_{2}=\pi / 2$, while on the boundary $\nu=-\epsilon$, they merge with the values $\psi_{1}=-\pi / 2, \psi_{2}=3 \pi / 2 \equiv(-\pi / 2$ $\bmod 2 \pi$ ). At each point outside of the tongue (i.e., $|\nu|>\epsilon), \dot{\psi}$ cannot change sign; therefore, $\psi(t)$ is a strictly monotone function, either increasing or decreasing with $t$ depending on the sign of $\nu$; see subsection 6.1.2.

Now consider the eigenvalue $\lambda_{3}$ of the Jacobian $J_{1}$ in (80). In the notation of (82), this requires

$$
\lambda_{3}=-2 B_{2} \mu_{1} \cos (\theta+\phi) \equiv-2 \epsilon \cos \psi=0 .
$$

Note that $\lambda_{3}=0$ is the same as $\frac{d \dot{\psi}}{d \psi}=0$ in (84); that is, $\lambda_{3}=0$ defines the boundaries of the Arnold tongue for the Adler equation, where the two phase equilibria $\psi_{1}, \psi_{2}$ merge and vanish in a saddle-node bifurcation. As remarked in section 6.1, this saddle-node bifurcation in $\theta$ on the normal mode branch with $r_{2}=0$ is a "phantom bifurcation" for the original 4-dimensional system, since it occurs "inside the point" $r_{2}=0$ and is not observable. We return to this paradox of bifurcation in $\theta$ after exploring a pitchfork bifurcation that can lead to $r_{2}>0$.

In the case $\dot{\psi}=0$ in (84) (and thus $\psi=\psi_{1}$ or $\psi_{2}$ ), squaring and adding the two equations (83) and (84) to eliminate the trigonometric functions gives a right circular cone with its axis along the $\epsilon$-axis in three dimensions:

$$
\delta^{2}+\nu^{2}=\epsilon^{2}
$$

Note that in the 3 -dimensional $(\epsilon, \delta, \nu)$ parameter space, the boundaries $\nu= \pm \epsilon$ of the Arnold tongue are tangent planes touching the cone (86) in the lines $\{\delta=0, \nu= \pm \epsilon\}$. The singular behavior on these lines of contact is studied in subsection 6.1.3.

Now consider the conditions for a phase-locked secondary bifurcation at $\lambda_{2}=0$ on the primary branch $\left\{r_{1}=\sqrt{\mu_{1}}, r_{2}=0\right\}$ for fixed $\mu_{1}>0$; that is, the simultaneous solution of (81) and (79), or equivalently of (83) and (84), with $\theta \equiv \theta_{0}$ and $\dot{\theta}=\dot{\psi}=0$. This secondary bifurcation occurs as $\delta$ crosses the circle (86) transversally for fixed $\epsilon$. However, at the top and bottom of the cones $\{\delta=0, \nu= \pm \epsilon\}$, this path is a tangent to the cone, and the "crossing" is degenerate. Furthermore, as shown in subsection 6.1.3 and confirmed numerically in section 6.3 , codimension-two bifurcations exist at these top and bottom points, which give rise to additional secondary bifurcations nearby. Therefore in this subsection we must exclude neighborhoods of each of these two points $(\delta=0, \nu= \pm \epsilon)$ for fixed $\epsilon>0$. That is, we postulate a small parameter $\delta_{1}>0$, which is small compared to $\epsilon$ but is large enough 
to exclude the neighborhood of the codimension-two bifurcation, and consider only points on the cone (86) satisfying $|\delta|=\sqrt{\epsilon^{2}-\nu^{2}}>\delta_{1}$. With these restrictions, there are two such crossings and they are transverse. At $\delta=-\sqrt{\epsilon^{2}-\nu^{2}}<-\delta_{1}$, the corresponding equilibrium point of $(84)$ is $\psi_{1} \in\left(-\frac{\pi}{2}, \frac{\pi}{2}\right)$. As noted above, this equilibrium is asymptotically stable for the phase equation (84). At $\delta=+\sqrt{\epsilon^{2}-\nu^{2}}>\delta_{1}$, the corresponding equilibrium point of (84) is $\psi_{2} \in\left(\frac{\pi}{2}, \frac{3 \pi}{2}\right)$, and this equilibrium is unstable for (84). Now returning to the 3 -dimensional truncated normal form equation (53), it is clear that these two bifurcations are supercritical pitchforks, in the sense that $r_{2}>0$ for $\mu_{2}$ (or $\delta$ ) increasing past the bifurcation point. The equilibrium phase $\psi_{1}$ of (84) corresponds to the bifurcation of an equilibrium point $\left(r_{1}, r_{2}, \theta_{0}\right)$ with $r_{1} \gg r_{2}>0$ and $\theta_{0}=\psi_{1}-\phi$, and this point is asymptotically stable for $\delta>-\sqrt{\epsilon^{2}-\nu^{2}}$. Similarly, equilibrium phase $\psi_{2}$ also corresponds to the bifurcation of an equilibrium point $\left(r_{1}, r_{2}, \theta_{0}\right)$ with $r_{1} \gg r_{2}>0$ and $\theta_{0}=\psi_{2}-\phi$, but for $\delta>+\sqrt{\epsilon^{2}-\nu^{2}}$, and this equilibrium point is unstable. In other words, a stable equilibrium point of (53) is born as $\delta$ enters the circle (86) from the left, and a second unstable equilibrium point is born as $\delta$ leaves the circle (86) on the right, provided that these crossings lie outside of the two excluded neighborhoods.

Finally, consider the 4-dimensional normal form equation (54) in polar coordinates $\left(r_{1}, r_{2}\right.$, $\left.\theta_{1}, \theta_{2}\right)$. Each of the two equilibrium points of the 3-dimensional normal form (78) with $r_{1} \gg$ $r_{2}>0$ represents an orbit of (54) for which both normal modes are "switched on." Since $0=\dot{\psi}=\dot{\theta}=2\left(\dot{\theta}_{1}-\dot{\theta}_{2}\right)$, the two normal modes are synchronized; that is, they have the same angular frequencies and a fixed phase difference. Therefore, the two secondary pitchfork bifurcations of the previous paragraph give rise to phase-locked mixed mode periodic solutions in the 4-dimensional state space.

As noted above, these two pitchfork bifurcations occurring on $\delta= \pm \sqrt{\epsilon^{2}-\nu^{2}}$, away from $\delta=0$, are both supercritical in the sense that each has $r>0$ for $\delta$ to the right of its respective bifurcation curve. Therefore, the bifurcation on $\delta=-\sqrt{\epsilon^{2}-\nu^{2}}$ has $r>0$ inside the cone, while the bifurcation on $\delta=+\sqrt{\epsilon^{2}-\nu^{2}}$ has $r>0$ outside the cone. This raises a natural question. Does the bifurcation branch "flip" from inside to outside the cone, in a neighborhood of the points $\delta=0, \nu= \pm \epsilon$ at the top and bottom of the cone? The answer to this question involves the codimension-two bifurcations studied in subsections 6.1.3 and section 6.3.

Now return to the paradox of the "phantom" saddle-node bifurcation in $\theta$, which is undefined in the 4-dimensional normal form equation (54). A prerequisite for the existence of the phase-locked mixed mode periodic solutions of the previous paragraph is the existence of phase equilibria $\theta_{0}=\psi_{j}-\phi(j=1,2)$ for the 3 -dimensional normal form (53). These phase equilibria exist only inside the Arnold tongue. Therefore, the Arnold tongue defines a region in which the secondary pitchfork bifurcation to $r_{2}>0$ can give rise to phase-locked periodic solutions. On crossing to the outside of the Arnold tongue, the phase equilibria $\theta_{0}$ disappear in a saddle-node bifurcation, and there is no bifurcation to a phase-locked mixed mode periodic solution. The behavior of the system outside of the Arnold tongue is the subject of the next section.

6.1.2. Bifurcation of quasi-periodic solutions from the in-phase normal mode. In this subsection, assume that the unfolding parameters lie outside of the Arnold tongue in Figure 4; that is, $|\nu|>\epsilon>0$. There are two cases: either $\nu>\epsilon$ and $\dot{\psi}>0$, or $\nu<-\epsilon$ and $\dot{\psi}<0$. In these cases $\psi(t)$ is strictly monotone increasing in $t$ or $-t$, respectively; that is, $\psi$ is a time- 
like variable. Consider here the first case; the second is similar. Under these assumptions, we study secondary bifurcation from the in-phase normal mode and show that it leads to quasi-periodic solutions on a toroidal breather, as investigated in section 5 .

Bifurcation from a periodic solution occurs when a corresponding Floquet multiplier crosses the unit circle. These Floquet multipliers are the eigenvalues of the linearization of the Poincaré map defined by the vector field, in a neighborhood of the known periodic solution. The Poincaré map of a nonlinear system is difficult to calculate. However, this difficulty may be overcome by means of the following well-known fact $[17,28]$. The linearization of the Poincaré map of a nonlinear vector field about a known periodic orbit is the same as the Poincaré map of the linearization of the vector field about that orbit. The latter is much easier to calculate.

Return to the 3 -dimensional system $(78)$ in $\left(r_{1}, r_{2}, \theta\right)$. In the present case, $\dot{\theta}>0$ and $\dot{\theta}$ is bounded away from zero in some neighborhood of the in-phase periodic solution, so we can use $\theta$ as the "time variable" and reduce the 3 -dimensional system to a nonautonomous 2 -dimensional system as in section 5 , that is,

$$
\begin{aligned}
\frac{d r_{1}}{d \theta} & =\frac{r_{1}\left[\mu_{1}-r_{1}^{2}+\left(b_{12}+B_{1} \cos \theta\right) r_{2}^{2}\right]}{D(\theta)} \\
\frac{d r_{2}}{d \theta} & =\frac{r_{2}\left[\mu_{2}+\left(b_{21}+B_{2} \cos (\theta+\phi)\right) r_{1}^{2}-r_{2}^{2}\right]}{D(\theta)},
\end{aligned}
$$

where

$$
D(\theta)=2\left[\mu_{3}+\left(b_{31}-B_{2} \sin (\theta+\phi)\right) r_{1}^{2}+\left(b_{32}-B_{1} \sin \theta\right) r_{2}^{2}\right]
$$

and we verify below that $D(\theta)>0$. Then (87) has equilibrium solution $\left(r_{1}, r_{2}\right)=\left(\sqrt{\mu_{1}}, 0\right)$. Translate to new coordinates $\left(r_{1}, r_{2}\right)=\left(\sqrt{\mu_{1}}+v_{1}, v_{2}\right)$ and linearize (87) at the new origin. Also, simplify the parameters and phase as in (82). The result is

$$
\left[\begin{array}{l}
v_{1}^{\prime} \\
v_{2}^{\prime}
\end{array}\right]=\frac{1}{D_{0}(\psi)}\left[\begin{array}{cc}
-2 \mu_{1} & 0 \\
0 & \delta+\epsilon \cos \psi
\end{array}\right]\left[\begin{array}{l}
v_{1} \\
v_{2}
\end{array}\right] \equiv A(\psi)\left[\begin{array}{l}
v_{1} \\
v_{2}
\end{array}\right]
$$

where

$$
D_{0}(\psi)=2[\nu-\epsilon \sin (\psi)], \quad v_{j}{ }^{\prime} \equiv \frac{d v_{j}}{d \psi}, j=1,2 .
$$

Here $D_{0}(\psi)>0$ follows from the assumption $\nu>\epsilon>0$, and therefore $D(\theta)>0$ also holds in (88), for $\left(r_{1}, r_{2}\right)$ in a suitable neighborhood of $\left(\sqrt{\mu_{1}}, 0\right)$. The coefficient matrix $A(\psi)$ in $(89)$ is $2 \pi$-periodic in $\psi$, so that Floquet theory applies. This system is diagonal, which makes it easy to solve. In the subspace $\left\{\left(v_{1}, 0\right)\right\}$,

$$
\frac{d v_{1}}{d \psi}=\left[\frac{-\mu_{1}}{\nu-\epsilon \sin \psi}\right] v_{1}
$$

has the solution

$$
v_{1}(\psi)=v_{1}(0) \cdot \exp \left[-\frac{\mu_{1}}{\nu} \int_{0}^{\psi} \frac{d s}{1-\alpha \sin s}\right]
$$


where $0<\alpha \equiv \epsilon / \nu<1$. From this we obtain a Floquet multiplier in the $v_{1}$ direction as

$$
\frac{v_{1}(2 \pi)}{v_{1}(0)}=\exp \left[-\frac{\mu_{1}}{\nu} \int_{0}^{2 \pi} \frac{d s}{1-\alpha \sin s}\right] .
$$

Since this integral is always positive, the Floquet multiplier has modulus less than one, and this implies asymptotic stability in the $v_{1}$ (or $r_{1}$ ) direction, as expected. The corresponding Floquet exponent is

$$
\chi_{1}=-\frac{\mu_{1}}{2 \pi \nu} \int_{0}^{2 \pi} \frac{d \psi}{1-\alpha \sin \psi},
$$

which is always negative. Similarly, for the second Floquet exponent $\chi_{2}$, we have

$$
\frac{d v_{2}}{v_{2}}=\left[\frac{\delta+\epsilon \cos \psi}{2 \nu(1-\alpha \sin \psi)}\right] d \psi
$$

which we integrate to obtain

$$
\chi_{2}=\frac{\delta}{4 \pi \nu} \int_{0}^{2 \pi} \frac{d \psi}{1-\alpha \sin \psi}
$$

since the second term in the integrand of (91) integrates to zero. The integral is always positive as above, and therefore the Floquet exponent $\chi_{2}$ is zero if and only if

$$
\delta \equiv \mu_{2}+\mu_{1} b_{12}=0 .
$$

The Floquet exponent $\chi_{2}$ crosses from negative to positive as $\delta$ does, so it follows that the in-phase normal mode solution is asymptotically stable for $\delta<0$ and unstable for $\delta>0$, undergoing a bifurcation at $\delta=0$. For the assumed case of $a_{22}<0$, it is clear from (87) that this is a supercritical pitchfork bifurcation; that is, a solution exists with $r_{2}>0$ when $\delta>0$. But $r_{2}>0$ implies that $\theta_{2}$ is defined, and then, since $\dot{\theta} \equiv \dot{\theta_{1}}-\dot{\theta_{2}}>0$, in this case the in-phase and anti-phase modes cannot phase-lock. The solution in this case is quasi-periodic with two primary frequencies of order one and a third low frequency "modulation" of $r_{1}, r_{2}$, that is, a toroidal breather, as found in section 5 .

In the $(\epsilon, \delta, \nu)$ parameter space, the bifurcation plane $\delta=0$ intersects the cone (86) exactly at the top and bottom where $\nu= \pm \epsilon$, that is, where the boundary of the Arnold tongue touches the cone. The behavior near this degenerate line of intersection is explored in the next subsection.

6.1.3. Codimension-two secondary bifurcations from the in-phase mode. As noted in subsection 6.1, the Jacobian (80) may have a double zero eigenvalue, $\lambda_{2}=\lambda_{3}=0$. Generically, a double zero eigenvalue leads to the Bogdanov-Takens bifurcation. However, that is not the case here. As can be seen from (80), the double zero eigenvalue here is semisimple, instead of nonsemisimple as required for the Bogdanov-Takens case. This is a consequence of the pitchfork symmetry for $r_{2}$ in (53), which in turn is a consequence of the fact that $r_{2}$ is the amplitude of a periodic oscillation. Therefore, one may anticipate that the normal form of this 
codimension-two secondary bifurcation corresponds to that of the classical codimension-two saddle-node-Hopf (or fold-Hopf) bifurcation, reduced to two dimensions by the suppression of the phase variable, since both have the same symmetry; see [9, 16, 25, 38].

From (80), it is clear that $\lambda_{3}=0$ only if $\cos \psi=0$ (where $\psi \equiv \theta+\phi$ as in (82)), and then $\psi= \pm \pi / 2$. In this case, $\lambda_{2}=0$ if and only if $\delta \equiv \mu_{2}+b_{21} \mu_{1}=0$. Therefore, $\lambda_{2}=\lambda_{3}=0$ at the top and bottom points on the cone (86), i.e., the maximum values of $|\nu|$ for fixed $\epsilon>0$. At these points, the third eigenvalue is $\lambda_{1}=-2 \mu_{1}=-2 r_{1}^{2}=\mathcal{O}(\epsilon)$, which is bounded away from zero by hypothesis. Consider here the case $\psi=\pi / 2$ at the top of the cone; the case $\psi=-\pi / 2$ is similar. Apply the center manifold theorem to reduce the 3 -dimensional system (53) locally to a 2 -dimensional system in $r_{2}, \psi$. Next change the parameters $\left(\mu_{1}, \mu_{2}, \mu_{3}\right)$ as in (82). We make explicit the fact that, near the point of secondary bifurcation, $r_{2}$ is small compared to $r_{1}$, by substituting $r_{2}=r \sqrt{\epsilon}$. The resulting system is

$$
\begin{aligned}
\dot{r} & =r\left[\delta+\epsilon \cos (\psi)-\epsilon r^{2}\right], \\
\dot{\psi} & =2\left[\nu-\epsilon \sin (\psi)+\left(b_{32}-B_{1} \sin (\psi-\phi)\right) \epsilon r^{2}\right] .
\end{aligned}
$$

Now write $\psi=\pi / 2+z$ and expand $\cos \psi, \sin \psi$ in a Taylor series about $\psi=\pi / 2$; that is, $\cos (\pi / 2+z)=-\sin z=-z+\cdots$ and $\sin (\pi / 2+z)=\cos z=1-z^{2} / 2+\cdots$. Finally, divide through by $\epsilon \neq 0$, rescale $t$ by $\epsilon$ (slow time), and introduce new small unfolding parameters $\epsilon_{1}=\delta / \epsilon$ and $\epsilon_{2}=2(\nu-\epsilon) / \epsilon$. Then (93) locally becomes

$$
\begin{aligned}
& \dot{r}=r\left[\epsilon_{1}-z-r^{2}\right], \\
& \dot{z}=\epsilon_{2}+z^{2}+\eta r^{2},
\end{aligned}
$$

where

$$
\eta \equiv 2\left[b_{32}-B_{1} \sin \left(\frac{\pi}{2}-\phi\right)\right]
$$

and we assume $\eta \neq 0$. As expected, system (94) has the same form as the 2-dimensional normal form for the well-known codimension-two saddle-node-Hopf bifurcation; see [9, 16, 25, 38]. These references present the complete set of bifurcation diagrams and phase portraits for that case, which will not be repeated here. However, these different authors use different choices of notation in their normal forms. For consistency in the following, we refer only to the normal forms and diagrams of Kuznetsov [25, Chapter 8] for the saddle-node-Hopf bifurcation. System (94) corresponds to the normal form (8.82) in [25] with the transformations $(r, z) \rightarrow(\rho, \xi)$, $\left(\epsilon_{1}, \epsilon_{2}\right) \rightarrow\left(\beta_{2}, \beta_{1}\right), \theta=-1$, and $s=\operatorname{sign}(\eta)$.

There are some fundamental distinctions that must be made between the present case (94) of double zero bifurcation and the classical saddle-node-Hopf case, which we enumerate as follows:

1. The state space of the variable $z \equiv \psi-\pi / 2$ here is $\mathrm{S}^{1}$, not $\mathbb{R}^{1}$. The state variable $r$ is in $\mathbb{R}^{+}$for both cases. Therefore, the phase portraits represented in a half-plane in $[9,16,25,38]$ must be wrapped around a half-cylinder $\mathbb{R}^{+} \times \mathrm{S}^{1}$ for the present case. In the saddle-node-Hopf case, most solutions grow unbounded as $|z| \rightarrow \infty$, but these will return with period $2 \pi$ in $z$ in the present case. 
2. The saddle-node bifurcation on the $z$-axis where $r=0$ in (94) is a "phantom" bifurcation for our original system, as explained in section 6.1. Even so, the codimension-two bifurcation (94) serves to organize the behavior of solutions for small $r_{2}>0$.

3. There is a pitchfork bifurcation in system (94) that occurs as the parameters cross the parabola $\epsilon_{2}=-\epsilon_{1}^{2}$. (This parabola is a local approximation to the top of the inphase cone described in section 6.1.) The bifurcation gives birth to a pair of equilibria $\left( \pm r_{0}, z_{0}\right), r_{0}>0$. In the case of the saddle-node-Hopf bifurcation [25], this pitchfork bifurcation is actually a Hopf bifurcation of a periodic orbit. In the present case, this secondary pitchfork bifurcation implies both $r_{1}>0$ and $r_{2}>0$; that is, both normal modes are activated. However, at this equilibrium, also $\dot{z}=0$, which implies that the two normal modes are phase-locked with a single frequency. Thus the pitchfork bifurcation in (94) gives rise to a phase-locked mixed mode periodic solution.

In the saddle-node-Hopf case, the direction of the Hopf bifurcation (subcritical or supercritical) is determined by the coefficient $s$ in [25] corresponding to $\eta$ in (94). For the present case, rather than using the labels subcritical and supercritical, we call the pitchfork bifurcation "inward" if $r>0$ exists in the interior of the cone and "outward" if $r>0$ exists outside the cone. It follows that, for (94), the pitchfork bifurcation is "inward" if $\eta>0$ and "outward" if $\eta<0$.

This leads to an interesting paradox. Consider a neighborhood of the codimension-two point at the top of the in-phase cone, with $\eta<0$, where the pitchfork bifurcation is outward. However, on the left side of the cone, as shown in the previous section, the pitchfork bifurcation is inward. To resolve this paradox, the direction of the pitchfork must flip from inward to outward, near the top of the cone, on the left. The generic mechanism for this type of behavior is Bautin's bifurcation. The same argument implies a Bautin bifurcation on the right side of the bottom point of the cone.

4. System (94) does not exhibit the full range of behaviors of the classical saddle-nodeHopf normal form in references [9, 16, 25, 38], which all conclude that there are four distinct cases, determined by the sign of the coefficient $\eta$ in (94) and whether the $r z$ and $r^{2}$ terms have the same or opposite signs. Due to the special form imposed by the Huygens symmetry, the $r z$ and $z^{2}$ terms in (94) have coefficients that are equal in magnitude but opposite in sign. Therefore, of the four distinct cases that can exist in the generic case, only two may occur in the case (94). In the notation of Kuznetsov [25], system (94) corresponds to the case with $\theta=-1$, where $\theta$ is the coefficient of the $r z$ term. Therefore, the bifurcation diagrams and phase portraits for (94) are as shown in [25]: their Figure 8.14 for $\eta<0$ and Figure 8.16 for $\eta>0$, but the cases in their Figures 8.13 and 8.17 cannot occur for (94).

5. In two of the four cases presented in $[9,16,25,26,38]$, the topological behavior of the solutions of the normal form equations is determined by the quadratic terms only. In the remaining two cases the quadratic system has a degenerate secondary Hopf bifurcation, and cubic terms must be included to remove this degeneracy. It is proven in $[9,16,25]$ (using Gavrilov's transformation) that generically three of the four possible cubic terms may be eliminated, in such a way that the remaining one is sufficient to determine (topologically) the phase portraits in the degenerate case. The four possible cubic terms in the saddle-node-Hopf case are $r z^{2}, r^{3}$ in the $\dot{r}$ equation 
and $r^{2} z, z^{3}$ in the $\dot{z}$ equation. The Huygens symmetry in the present case forces three of these four cubic terms to be zero, leaving only the $r^{3}$ term in the $\dot{r}$ equation, as in (94). Previous authors have not considered this case. However, an application of the results in $[9,16,25]$ to the system (94) shows that the $r^{3}$ cubic term in (94) is sufficient to remove this case of degeneracy in the truncated quadratic system. This case occurs only for $\eta>0$ in (94) and behaves as shown in Figure 8.16 of [25]. For (94), the secondary Hopf bifurcation gives birth to a periodic orbit $(r(t), z(t))$ with a small frequency, surrounding the pitchfork equilibrium point $\left(r_{0}, z_{0}\right), r_{0}>0$. In the saddle-node-Hopf case, this periodic orbit corresponds to a 2-torus of quasi-periodic orbits surrounding the periodic orbit from the primary Hopf bifurcation, and the periodic orbit grows rapidly to a heteroclinic orbit and then "bursts," existing only very locally in the codimension-two unfolding. In the present case, this "secondary Hopf bifurcation" would give rise to a slow quasi-periodic modulation of the mixed mode phase-locked periodic solution created in the pitchfork bifurcation in item 3 .

As a concrete example to illustrate the above general theory, take parameter values as chosen in Figure 5 of section 6.3. First consider the point at the top of the cone for the in-phase primary bifurcation (cone on the left in Figure 5), where $\psi=\pi / 2, \nu=\epsilon>0$, and $\eta=-1.51$. Applying the above facts at this point, one finds a codimension-two secondary bifurcation with bifurcation diagram and phase portraits as in Figure 8.14 in [25]. In this case, locally the pitchfork bifurcation has $r>0$ outside the cone, and there is no "secondary Hopf" bifurcation. The discussion in item 3 above predicts a Bautin bifurcation to the left of the double zero bifurcation point. These theoretical predictions are confirmed by the numerical analysis in section 6.3 .

For the codimension-two point at the bottom of the in-phase cone in Figure 5, we have $\nu=-\epsilon<0$ and $\psi=(-\pi / 2 \bmod 2 \pi)$. The change of $\psi$ by $\pi$ reverses the signs of both the $r z$ and $z^{2}$ terms in (94). In order for these terms to match the normal form in [25], we transform $z \rightarrow-z$ in (94) (and hence $\theta \rightarrow-\theta$ ). Then the system (94) becomes

$$
\begin{aligned}
& \dot{r}=r\left[\epsilon_{1}-z-r^{2}\right], \\
& \dot{z}=-\epsilon_{2}+z^{2}+\eta r^{2},
\end{aligned}
$$

where now we have redefined $\epsilon_{2} \equiv 2(\nu+\epsilon) / \epsilon$ and $\eta \equiv-2\left[b_{32}-B_{1} \sin (-\pi / 2-\phi)\right]$. For the parameter values in Figure $5, \eta=+1.29$. This corresponds to the case depicted in Figure 8.16 of [25], with the substitution $\left(\epsilon_{1}, \epsilon_{2}\right) \rightarrow\left(\beta_{2},-\beta_{1}\right)$. Locally, the pitchfork bifurcation has $r>0$ inside the cone, and there does exist a "secondary Hopf" bifurcation with small frequency inside the cone, as confirmed by the numerical analysis in section 6.3. In addition, the numerical analysis finds a Bautin degenerate bifurcation to the right of the double zero bifurcation point, as suggested by the normal form analysis in this subsection.

6.2. Secondary bifurcation from the anti-phase normal mode. The case of secondary bifurcation from the anti-phase solutions is entirely analogous to that for the in-phase case presented in Section 6.1. On the primary anti-phase branch $r_{1}=0, r_{2}=\sqrt{\mu_{2}}>0$, the Jacobian of the truncated normal form (78), analogous to (80), is

$$
J_{2}=\left[\begin{array}{ccc}
\mu_{1}+\left(b_{12}+B_{1} \cos \theta_{0}\right) \mu_{2} & 0 & 0 \\
0 & -2 \mu_{2} & 0 \\
0 & 4 \sqrt{\mu_{2}}\left(b_{32}-B_{1} \sin \left(\theta_{0}\right)\right) & -2 B_{1} \mu_{2} \cos \left(\theta_{0}\right)
\end{array}\right] .
$$


The eigenvalues $\left\{\lambda_{1}, \lambda_{2}, \lambda_{3}\right\}$ may be read off the diagonal of $J_{2}$ as before for $J_{1}$. On the anti-phase normal mode branch, $\lambda_{2} \equiv-2 \mu_{2}<0$, implying stability in the $r_{2}$ direction. A secondary bifurcation that gives rise to a solution with $r_{1}>0$ may occur when

$$
\lambda_{1} \equiv \mu_{1}+b_{12} \mu_{2}+B_{1} \mu_{2} \cos \left(\theta_{0}\right)=0,
$$

where $\theta_{0}$ is an equilibrium point of the $\dot{\theta}$ phase equation in (53), which simplifies on the anti-phase branch $\left(r_{1}, r_{2}\right)=\left(0, \sqrt{\mu_{2}}\right)$ to

$$
\dot{\theta}=2\left(\mu_{3}+b_{32} \mu_{2}\right)-2 B_{1} \mu_{2} \sin (\theta) .
$$

Define new parameters in (98) by the nonsingular transformation analogous to (82),

$$
\left[\begin{array}{l}
\epsilon \\
\delta \\
\nu
\end{array}\right]=\left[\begin{array}{lll}
1 & b_{12} & 0 \\
0 & B_{1} & 0 \\
0 & b_{32} & 1
\end{array}\right]\left[\begin{array}{l}
\mu_{1} \\
\mu_{2} \\
\mu_{3}
\end{array}\right] .
$$

Then the equations corresponding to (83) and (84) are

$$
\begin{gathered}
0=\epsilon+\delta \cos \left(\psi_{0}\right), \\
\dot{\psi}=\nu-\delta \sin (\psi),
\end{gathered}
$$

where (102) is again the Adler equation, which exhibits Arnold tongues of stable/unstable pairs of equilibria (with $\dot{\psi}=0$ ) for $|\nu|<\delta$ in the $(\nu, \delta)$-plane. All of the secondary bifurcation phenomena described in section 6.1 are duplicated here for the anti-phase primary branch of solutions, but with $\delta$ and $\epsilon$ interchanged. Inside the Arnold tongue, secondary bifurcation of phase-locked mixed mode periodic solutions occurs on crossing the right circular cone with its axis along the $\delta$-axis,

$$
\epsilon^{2}+\nu^{2}=\delta^{2}
$$

Outside of the Arnold tongue, there exists secondary bifurcation to quasi-periodic solutions on a toroidal breather, as in subsection 6.1.2.

Equations (86) and (103) represent right circular cones with orthogonal axes in the $(\epsilon, \delta, \nu)$ coordinates; however, in the original $\left(\mu_{1}, \mu_{2}, \mu_{3}\right)$ parameters these cones are linearly transformed by (82) and (100). The analysis in sections 6.1-6.2 is local to a neighborhood of each of the secondary bifurcations. Therefore, it cannot describe the behavior between the two cones or deal with the case when the two cones intersect. For a more global understanding of the connections between these two local secondary bifurcations, we turn to numerical analysis.

6.3. Numerical study of secondary bifurcations. We use numerical analysis of the normal form equations (53) to extend the local analytical results of the previous subsections and to show how they are connected with the periodic and quasi-periodic solutions of sections 4 and 5 . These results are expressed in terms of the original normal form parameters $\left(\mu_{1}, \mu_{2}, \mu_{3}\right)$. In addition, we discuss these bifurcations in terms of the 4-dimensional system (54).

The cubic truncated normal form (53) is scale-invariant in the following sense. Given a positive factor $g$, scaling the parameters $\mu_{1}, \mu_{2}$, and $\mu_{3}$ by $g$, scaling time $t$ by $1 / g$, and 
scaling the state variables $r_{1}$ and $r_{2}$ by $\sqrt{g}$ yields an identical system. Therefore, away from the origin in the $\mu$-parameter space we may scale by $g=1 / \sqrt{\mu_{1}^{2}+\mu_{2}^{2}+\mu_{3}^{2}}$, showing that it is enough to consider values of $\mu$ on the unit sphere centered at the origin. In addition, any bifurcations occurring at points on this sphere also occur at all points on the rays connecting these points to the origin. Algebraically, it is sometimes convenient to consider planes with $\mu_{i}$ a nonzero constant, $1 \leq i \leq 3$. Since all points on such a plane lie on exactly one ray from the origin, the bifurcation diagram on such a plane is topologically equivalent to that on an open hemisphere of the unit sphere.

In this section, we numerically compute the bifurcation varieties of the cubic truncated normal form (53) for secondary bifurcation from the normal mode solutions. The normal mode in-phase solution for $(54),\left(r_{1}, r_{2}\right)=\left(\sqrt{\mu_{1}}, 0\right)$, exists for $\mu_{1}>0$, and the anti-phase solution, $\left(r_{1}, r_{2}\right)=\left(0, \sqrt{\mu_{2}}\right)$, exists for $\mu_{2}>0$, but they are not stable for all parameter values. Consider the in-phase normal mode solution; the analysis for the anti-phase solution is analogous. Following the analysis of sections 6.1.1 and 6.1.2, we consider three cases for $\mu_{3}$, as defined by the Arnold tongue of section 6.1.1:

(i) $\mu_{3}>\left(-b_{31}+B_{2}\right) \mu_{1}$; that is, $\nu$ lies above the Arnold tongue.

(ii) $\mu_{3}<\left(-b_{31}-B_{2}\right) \mu_{1}$; that is, $\nu$ lies below the Arnold tongue.

(iii) $\left(-b_{31}-B_{2}\right) \mu_{1}<\mu_{3}<\left(-b_{31}+B_{2}\right) \mu_{1}$; that is, $\nu$ lies inside the Arnold tongue.

6.3.1. Secondary bifurcation to a toroidal breather. In case (i), the Floquet analysis of section 6.1.2 shows that the in-phase normal mode solution loses stability when $\delta$ increases through zero, or equivalently when $\mu_{2}$ crosses the half-plane,

$$
\mu_{2}+b_{21} \mu_{1}=0, \quad \mu_{1}>0
$$

in the positive direction. The half-plane (104) shall be called the "in-phase plane." The inphase plane is shown in yellow on the left in Figures 5 and 6 . For case (ii), we have $\dot{\theta}<0$, and the only difference from the above is that $\theta$ decreases monotonically in $t$; the "in-phase plane" is unaltered. A cross section of the plot in Figure 5 is shown in Figure 6.

In both cases (i) and (ii), that is, when $\left|\mu_{3}+b_{31} \mu_{1}\right|>B_{2} \mu_{1}$, when $\mu$ crosses the inphase plane (in the direction of increasing $\mu_{2}$ ), the Poincaré map for this periodic orbit has an eigenvalue increasing through unity, as shown in section 6.1.2. Since the equilibrium corresponding to the normal mode solution persists and the map is odd, the map undergoes a pitchfork bifurcation. The pitchfork has two nontrivial branches, but the solution on the second one has $r_{2}<0$ and is the symmetric pair to the solution from the first branch, as in Lemma 2.1, and they are related to each other by switching the two oscillators. We discuss only the branch yielding $r_{2}>0$. The solution on the nontrivial branch of the pitchfork is a periodic orbit for (53) with all three state variables oscillating. The type of pitchfork (superor subcritical) and the associated stability of this new solution are discussed below. When interpreted in terms of the 4-dimensional system (54), the bifurcation at the in-phase plane is a secondary Hopf bifurcation of the equilibrium point for the 3-dimensional Poincaré map for the in-phase normal mode periodic orbit on a hyperplane with constant $\theta_{1}$. The new solution born from this bifurcation is a quasi-periodic breather, and it lives on a 3-torus since the frequencies of $\theta_{1}, \theta_{2}$, and $\theta$ are not rationally related. 
6.3.2. Secondary bifurcation to periodic solutions. In case (iii), the parameters lie inside the Arnold tongue and the secondary bifurcations are as presented in section 6.1.1. We conclude that for case (iii), the in-phase normal mode solution is stable if $\mu_{2}$ is below the cone

$$
\left(\mu_{2}+b_{21} \mu_{1}\right)^{2}+\left(\mu_{3}+b_{31} \mu_{1}\right)^{2}=\left(B_{2} \mu_{1}\right)^{2}, \quad \mu_{1}>0 .
$$

This cone is called the "in-phase cone" and is shown in blue in Figure 5. It is the same as the cone (86) in Section 6.1.1.

The in-phase plane (104) of cases (i) and (ii) passes through the center line of the in-phase cone and intersects the cone at its top and bottom. The boundaries between cases (i) and (iii) and between (ii) and (iii) are precisely these intersections and are shown as magenta lines and circles in Figures 5 and 6.

In case (iii), the in-phase normal mode periodic solution of (54) is represented in system (53) not as a single periodic orbit but as two equilibria, $e_{1}$ and $e_{2}$ with different values of $\theta$, and the two orbits on the invariant curve $\left(r_{1}, r_{2}, \theta\right)=\left(\sqrt{\mu_{1}}, 0, \theta\right), \theta \in[0,2 \pi)$, that connect them. Indeed, the planes $\mu_{3}=-b_{31} \mu_{1} \pm B_{2} \mu_{1}$, which are tangent to the in-phase cones where the in-phase planes meet them, are saddle-node on an invariant circle bifurcations for system (53). These planes are not presented in Figure 5 since they are not bifurcations for the 4-dimensional system (54) but are rather the "phantom bifurcations" of section 6.1.1. Both equilibria are stable in the $r_{1}$ direction, but $e_{1}$ is stable in the $\theta$ direction while $e_{2}$ is not. For $\mu_{2}$ to the left of the in-phase cone (105) in Figure 5, both equilibria are stable in the $r_{2}$ direction. As $\mu$ crosses into the interior of the in-phase cone (105), the equilibrium $e_{1}$ undergoes a pitchfork bifurcation and becomes unstable in the $r_{2}$ direction. This bifurcation may be supercritical or subcritical, as described in section 6.3.3. As $\mu$ passes back to the exterior of the in-phase cone so that $\mu_{2}$ is now to the right of this cone in Figure 5, the second equilibrium, $e_{2}$, also loses stability in the $r_{2}$ direction via a pitchfork bifurcation, which again can be either super- or subcritical. The equilibrium of (53) that lies on the nontrivial branch of the pitchfork represents a phase-locked mixed mode periodic solution of system (54), where $r_{1}$ and $r_{2}$ are positive constants and $\theta_{1}$ and $\theta_{2}$ are varying but with constant difference. For (54) then, the in-phase cone marks a pitchfork bifurcation of the equilibrium point of the 3-dimensional Poincaré map for the in-phase normal mode periodic orbit on a hyperplane with constant $\theta_{1}$. For (53) the intersections of the in-phase plane and the in-phase cone are degenerate double zero bifurcations where a saddle-node on an invariant circle meets a pitchfork bifurcation of the in-phase equilibrium, as studied in section 6.1.3. For system (54) these bifurcations at the plane-cone intersections are degenerate double unity bifurcations of the equilibrium point for the 3-dimensional Poincaré map for the in-phase normal mode periodic orbit on a hyperplane with constant $\theta_{1}$.

The analogous conclusion for the anti-phase normal mode solution is that it is stable, provided that $\mu_{1}$ is below the "anti-phase plane"

$$
\mu_{1}+b_{12} \mu_{2}=0, \quad \mu_{2}>0
$$

if $\left|\mu_{3}+b_{32} \mu_{2}\right|>B_{1} \mu_{2}$, or provided that $\mu_{1}$ is below the "anti-phase cone"

$$
\left(\mu_{1}+b_{12} \mu_{2}\right)^{2}+\left(\mu_{3}+b_{32} \mu_{2}\right)^{2}=\left(B_{1} \mu_{2}\right)^{2}, \quad \mu_{2}>0,
$$




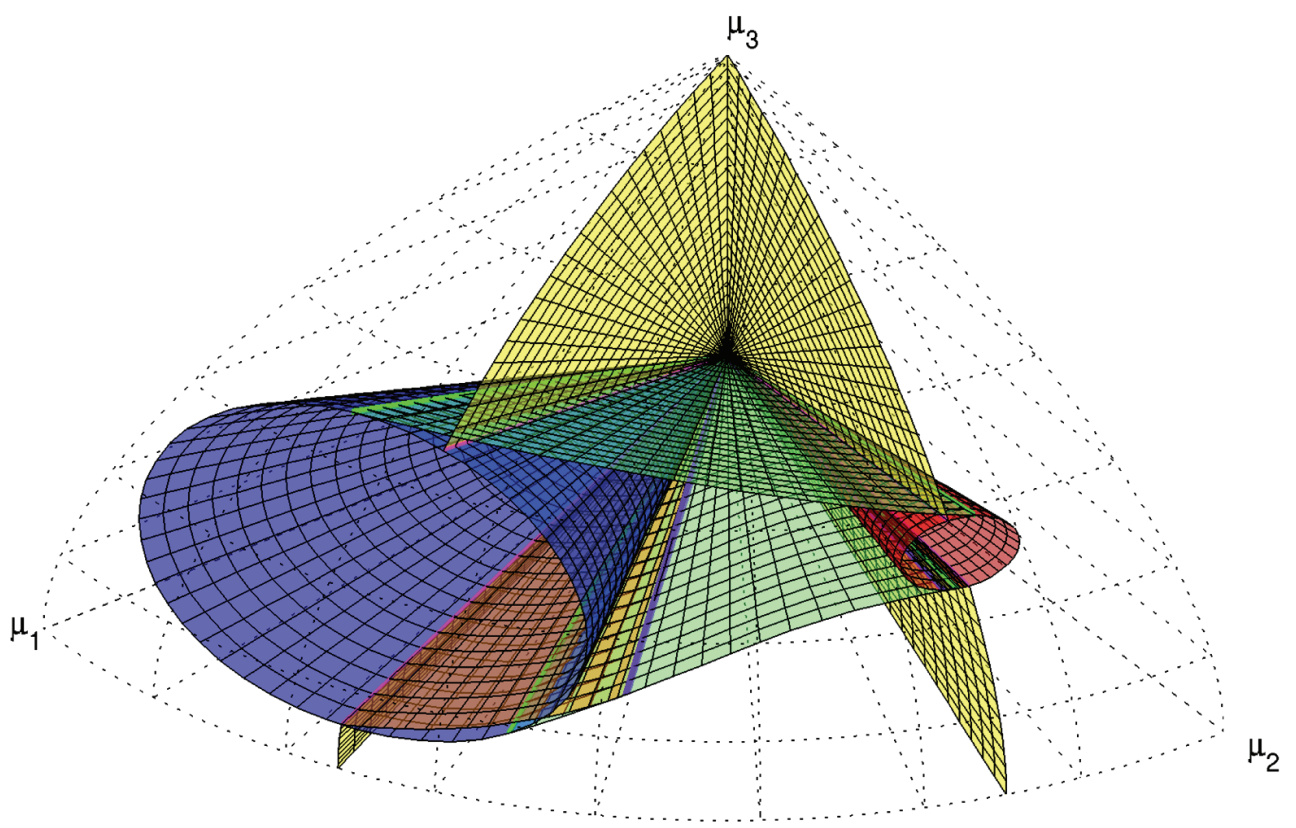

Figure 5. Secondary bifurcations for (53) in the $\mu$ parameter space in the case that the in-phase and anti-phase planes are regularly oriented, the cones do not intersect, and there are two mixed mode saddle-node surfaces that are smooth. The specific parameter values are $b_{12}=-0.3, b_{21}=-0.5, b_{31}=-0.6, b_{32}=-0.7$, $B_{1}=0.1, B_{2}=0.45$, and $\phi=1$. The yellow planar surfaces mark bifurcations of the normal mode solutions giving rise to quasi-periodic "breathers," which are locally stable and exist between these two planes. The blue and red cones are manifolds of bifurcations of the normal mode in-phase and anti-phase solutions, respectively, to mixed mode phase-locked periodic solutions. The green surfaces mark saddle-node bifurcations where two mixed mode phase-locked solutions coalesce. These surfaces intersect tangentially with the cones (green solid lines) in a degenerate pitchfork bifurcation for (53), determining the boundary of the out-facing and in-facing cone portions, which are colored slightly differently. The magenta lines, which are the intersections of the yellow planes with the cones, mark the location of a degenerate double zero bifurcation, and the purple lines on the green surfaces mark Bogdanov-Takens bifurcations. Surfaces of Hopf bifurcations (orange) connect the Bogdanov-Takens curves and the double zero curves. In the interior of the cones, one mixed mode phase-locked solution exists along with the normal mode solutions. Outside of the cones and between the two green surfaces, two mixed mode phase-locked solutions exist. Outside the region bounded by the cones and the saddle-node surfaces, no mixed mode phase-locked solutions exist.

if $\left|\mu_{3}+b_{32} \mu_{2}\right|<B_{1} \mu_{2}$. A quasi-periodic solution is born when $\mu$ crosses the anti-phase plane outside of the anti-phase cone, and a phase-locked mixed mode solution is born as $\mu$ crosses the surface of the anti-phase cone. The anti-phase cone is shown in red in Figures 5 and 6.

The above analysis reveals two distinct cases. Depending on the value of $b_{21}$, the in-phase plane (104) can lie anywhere in the region $\mu_{1}>0$, whereas the anti-phase plane (106) lies somewhere in the region $\mu_{2}>0$, depending on the value of $b_{12}$. These planes are parallel to the $\mu_{3}$-axis and intersect the $\left(\mu_{1}, \mu_{2}\right)$-plane in rays coming from the origin. Define the usual positive angle on the $\left(\mu_{1}, \mu_{2}\right)$-plane. If one starts with this angle at $-\pi / 2$ (the negative $\mu_{2}$-axis) and increases it, given random values for $b_{21}$ and $b_{12}$, one will most often encounter the in-phase plane before the anti-phase one. This shall be called the regular orientation of 


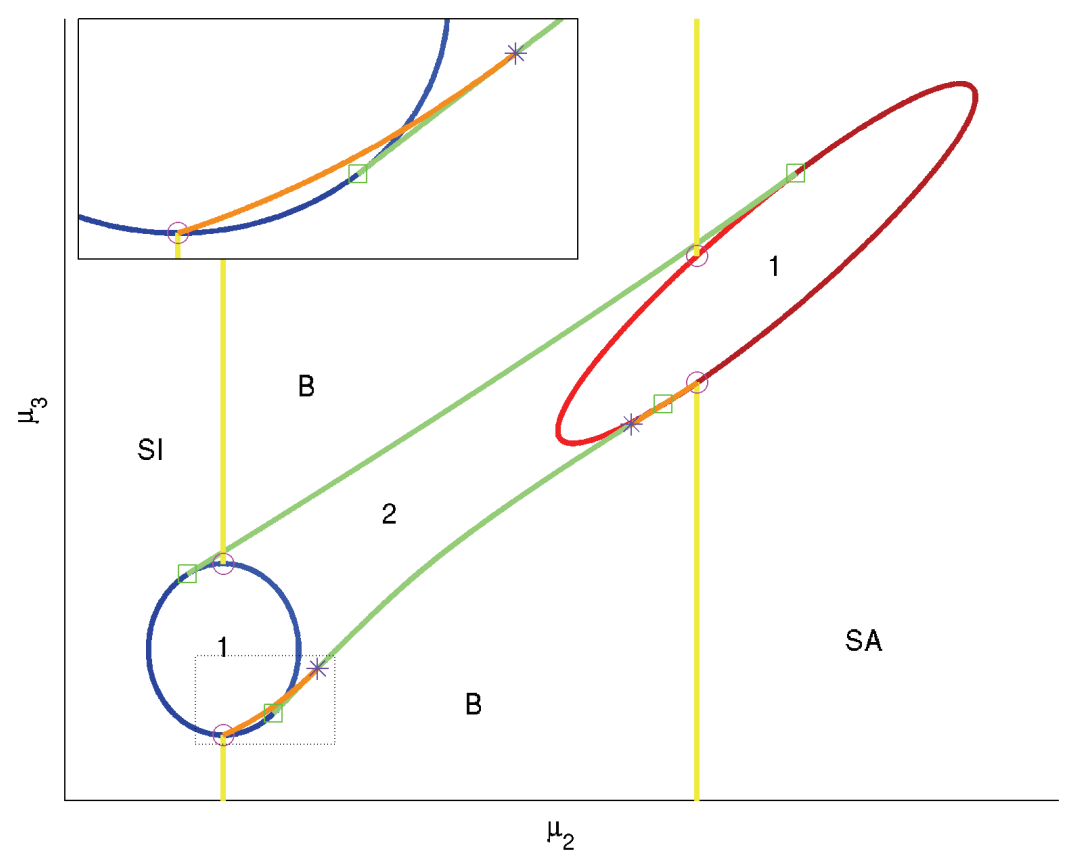

Figure 6. Cross section of the plot in Figure 5 along a plane with constant $\mu_{1}>0$. The colors correspond to those in the previous figure. The nonzero number of mixed mode phase-locked solutions in each region is indicated. The areas marked SI and SA are where stable in-phase and anti-phase, respectively, normal mode solutions exist. The areas marked $B$ are where the stable solutions are breathers. Squares mark the degenerate pitchfork bifurcations where the saddle-node surfaces intersect the cones, circles mark the double zero bifurcations at the extremes of the cones, and stars mark the Bogdanov-Takens bifurcations. The orange lines are Hopf bifurcations of a mixed mode solution. The inset shows a blow up of the region near the bottom of the in-phase cone marked by the dotted line.

these planes. However, if

$$
b_{12}<0, \quad b_{21}<0, \quad \text { and } \quad b_{12} b_{21}>1,
$$

then both planes are in the positive quadrant and, due to the last condition in (108), one encounters the anti-phase plane before the in-phase one. When (108) holds, we shall say the normal mode planes are in inverted orientation. If the planes are in the regular orientation, then between the two normal mode planes neither normal mode solution is stable. If the planes are in inverted orientation, then between the planes both the in-phase and anti-phase normal modes are simultaneously stable. In this case, the basins of attraction for the two normal modes change in size depending on how close $\mu$ is to the corresponding plane. If $\mu$ is closer to the in-phase plane than to the anti-phase plane, then the in-phase normal mode's basin of attraction is smaller. Numerical investigation causes us to conjecture that the pitchfork bifurcation of the Poincaré map for (53) for cases (i) and (ii) is supercritical if the planes are regularly oriented and is subcritical if the planes have inverted orientation. Consequently, 
breather solutions of (54) exist between the normal mode planes and are stable when the planes are regularly oriented but unstable when they are inverted. Mixed mode phase-locked solutions also interact with the breather solutions as discussed in the next section.

6.3.3. Bifurcations of the mixed mode solutions. The geometry of further bifurcations of the system depends strongly on the values of the parameters $b_{i j}$ and $B_{j}$. For now assume that these parameters are such that the cones do not intersect (and one is not inside the other) and the denominator of $h(\theta)$ given in (59) with $s_{1}=s_{2}=-1$ is always positive. Further, assume that the product $B_{1} B_{2}$ is sufficiently small so that $h(\theta)$ has at most two zeros (Remark 4.2).

Under the above restrictions, the equilibria for (53), which arose from the secondary bifurcations at the cone surfaces, disappear in a saddle-node bifurcation on a set of surfaces (the green surfaces in Figure 5 and green lines in Figure 6) that are tangent to and terminate at the two cones. In terms of system (54), at these surfaces a saddle-node of two mixed mode periodic orbits is occurring. These surfaces are defined by the requirements that $h(\theta)$, given by (59) with $s_{1}=s_{2}=-1$, and its derivative vanish simultaneously, so that a small perturbation causes this zero of $h$ to either disappear or split into two. In addition, the equilibrium values for $r_{1}^{2}$ and $r_{2}^{2}$, given by (58) with $s_{1}=s_{2}=-1$, at this zero of $h$ must be nonnegative. It is not possible to solve this system of equations analytically; however, by fixing say $\mu_{1}$, it is possible to solve the two equations for $\mu_{2}$ and $\mu_{3}$ in terms of $\theta$, yielding the following parametric representation of a curve lying on these surfaces:

$$
\begin{aligned}
D(\theta) & =1-\left(b_{12}+B_{1} \cos (\theta)\right)\left(b_{21}+B_{2} \cos (\theta+\phi)\right), \\
N_{1}(\theta) & =b_{31}-B_{2} \sin (\theta+\phi)+\left(b_{32}-B_{1} \sin (\theta)\right)\left(b_{21}+B_{2} \cos (\theta+\phi)\right), \\
N_{2}(\theta) & =b_{32}-B_{1} \sin (\theta)+\left(b_{31}-B_{2} \sin (\theta+\phi)\right)\left(b_{12}+B_{1} \cos (\theta)\right), \\
\mu_{2} & =\mu_{1} \frac{-N_{1}(\theta) D^{\prime}(\theta)+N_{1}^{\prime}(\theta) D(\theta)}{N_{2}(\theta) D^{\prime}(\theta)-N_{2}^{\prime}(\theta) D(\theta)}, \\
\mu_{3} & =-\frac{\mu_{1} N_{1}(\theta)+\mu_{2} N_{2}(\theta)}{D(\theta)} .
\end{aligned}
$$

The intersection of these saddle-node surfaces with the cones can be computed. This occurs when one of the equilibrium values $r_{10}$ or $r_{20}$ at the zero of $h$ becomes zero. Solving $h(\theta)=h^{\prime}(\theta)=0$, along with $r_{10}=\mu_{1}$ and $r_{20}=0$, after considerable simplification, the angle $\theta$ at which the surfaces intersect the in-phase cone is given by the solution of

$$
\begin{aligned}
\left(b_{12}+B_{1} \cos (\theta)\right)\left(b_{31} \sin (\theta+\phi)-b_{21} \cos (\theta+\phi)-B_{2}\right) & \\
& +\cos (\theta+\phi)+\left(b_{32}-B_{1} \sin (\theta)\right) \sin (\theta+\phi)=0 .
\end{aligned}
$$

This equation has between zero and four solutions (if $B_{1}$ and $B_{2}$ are sufficiently small, there are always exactly two solutions), which can then be used in (112) and (113) to determine the lines of intersection on the cone. The analogous equation for intersection with the anti-phase cone is

$$
\left(b_{21}+B_{2} \cos (\theta+\phi)\right)\left(b_{32} \sin (\theta)-b_{12} \cos (\theta)-B_{1}\right)+\cos (\theta)+\left(b_{31}-B_{2} \sin (\theta+\phi)\right) \sin (\theta)=0 .
$$


As shown in Figure 6, for the situation considered here, the saddle-node surfaces together with the cones bound a region in $\mu$-space wherein lie one or two mixed mode phase-locked solutions of (54). The intersections of the saddle-node surfaces with the cones divide the cones into two portions, an "out-facing" portion that is the boundary between regions with zero and one mixed mode solution, and an "in-facing" portion that is the boundary between regions with one and two mixed mode solutions. Whether the pitchfork bifurcation at a point on the cone surface is supercritical or subcritical depends on both the portion (in- or out-facing) that the point is on, and whether its $\mu_{2}$ or $\mu_{1}$ values are above or below the in-phase or anti-phase plane through the cone's center. For points on the out-facing portion of the in-phase cone and with $\mu_{2}$ below the in-phase plane, and for points on the in-facing portion of the in-phase cone and with $\mu_{2}$ above the in-phase plane, the bifurcation is supercritical. For all other points the bifurcation is subcritical. For the anti-phase cone the situation is the same but with $\mu_{2}$ replaced by $\mu_{1}$ in the above. Supercritical bifurcations from the out-facing portions of the cones give rise to stable mixed mode solutions, whereas supercritical bifurcations on the in-facing portions, although they transfer one dimension of stability from the normal mode to the mixed mode solution, still yield unstable mixed mode solutions since there is an instability in another direction. There are four rays on each cone surface that separate the super- and subcritical pitchfork bifurcations. For system (53), the intersections of the cones with the saddle-node surfaces are degenerate pitchfork bifurcations of the normal mode equilibria (solid green lines in Figure 5 and green squares in Figure 6), and the intersections of the in-phase (anti-phase) cones with the in-phase (anti-phase) planes are degenerate double zero bifurcations (magenta lines in Figure 5 and magenta circles in Figure 6). In terms of the 4-dimensional system (54), these are degenerate pitchfork and degenerate double unity bifurcations, respectively, of the equilibrium point of the 3-dimensional Poincaré map for the in-phase (anti-phase) periodic orbit on a hyperplane with constant $\theta_{1}\left(\theta_{2}\right)$.

A codimension-two Bogdanov-Takens bifurcation for system (53) can occur on the saddlenode surfaces, that is, a generic double zero eigenvalue of the Jacobian with a 1-dimensional eigenspace. Our investigations for the simple situation displayed in Figure 5 indicate that a Bogdanov-Takens bifurcation occurs on the saddle-node surface near the in-phase cone if the value of $\mu_{2}$ at the intersection of the saddle-node surface and the in-phase cone is above the inphase plane. A similar statement is true for Bogdanov-Takens points near the anti-phase cone, with $\mu_{2}$ replaced by $\mu_{1}$. The Bogdanov-Takens bifurcations are marked as purple lines and stars in Figure 5. A curve of Hopf bifurcations for system (53) emerges from the BogdanovTakens points tangent to the saddle-node surface and extends into the cone terminating at the degenerate double zero bifurcation (where the cone intersects the plane). The mixed mode solution that exists inside the cone is the one that undergoes Hopf bifurcation on this curve. The Hopf bifurcations are shown as orange surfaces and lines in Figures 5 and 6. Very nearby in parameter space to these Hopf bifurcations there appear to be homoclinic (to the unstable mixed mode equilibrium) or heteroclinic bifurcations (which connect the equilibria $e_{1}$ and $e_{2}$ ). Interpreted for system (54), these Hopf bifurcations are secondary Hopf bifurcations of the equilibrium point of the 3-dimensional Poincaré map for a mixed mode phase-locked periodic orbit on a hyperplane with constant $\theta_{1}\left(\theta_{2}\right)$. Initially, these orbits have a bounded value for $\theta$, but this is quickly lost when the heteroclinic/homoclinic bifurcation is met, and the orbit becomes a breather where $\dot{\theta}$ remains of one sign. 
A

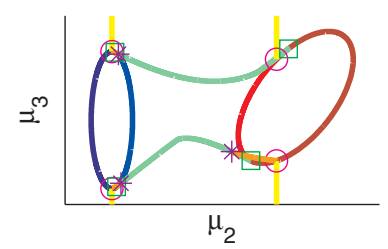

$\mathrm{D}$

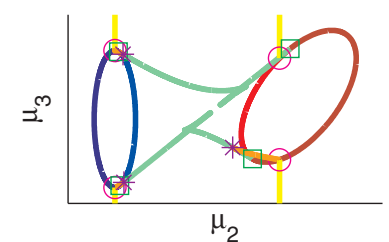

G

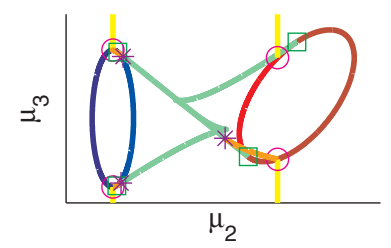

B

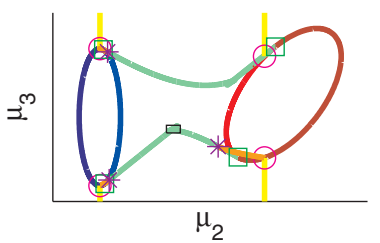

$\mathrm{E}$

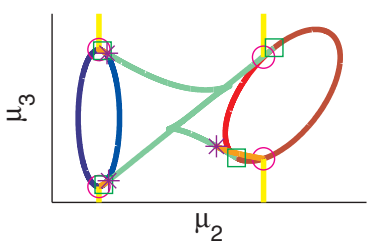

$\mathrm{H}$

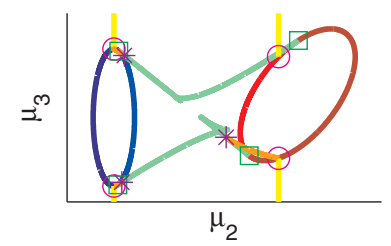

C

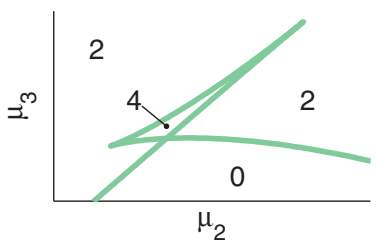

$\mathrm{F}$

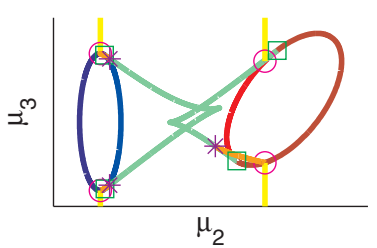

I

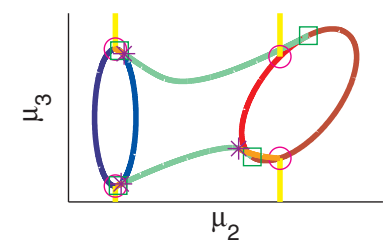

Figure 7. Bifurcation diagrams on a plane with constant $\mu_{1}$ showing existence of four mixed mode solutions, which come about through the generation of folds on the saddle-node (green) surfaces. Other symbols are as in Figure 6. The sequence shows this generation as $\phi$ is increased while $b_{12}=-0.3, b_{21}=0.25, b_{31}=-0.6$, $b_{32}=-0.2, B_{1}=0.1$, and $B_{2}=0.45$. A. $\phi=2.8$. A kink is just developing in the bottom surface; one will appear in the upper surface shortly. B. $\phi=3.0$. A swallowtail feature has developed in both surfaces. C. A blow up of the swallowtail feature from the area indicated by the black box in $B$. The number of mixed mode periodic solutions in each region is indicated. D. $\phi=3.095$. The swallowtails grow toward each other. E. $\phi=3.1$. The ends of the swallowtails meet, destroying themselves, and the surfaces exchange portions. F. $\phi=3.5$. Each branch of the saddle-node surface now clearly touches just one cone and has one cusp. G. $\phi=4.16$. The cusps have moved toward the other half of the other surface, and the situation is now the reverse of that in $\mathrm{E}$; the branches are about to reconnect and reform swallowtails. H. $\phi=4.2$. The surfaces have again exchanged portions so that they each again touch both cones; the swallowtail features are shrinking in size. I. $\phi=4.8$. The surfaces are again smooth, and there are no regions with four mixed mode solutions.

The saddle-node surfaces need not be smooth. If $h(\theta)$ has four solutions, this is manifested as folds in the saddle-node surfaces generating regions where four mixed mode solutions exist. Figure 7 shows a sequence of bifurcation diagrams on a plane with constant $\mu_{1}$ for different values of $\phi$. The saddle-node surfaces are the green curves in these cross sections. Initially each saddle-node surface is smooth, starts at the in-phase cone, and ends at the anti-phase cone (Figure 7A). Two mixed mode solutions exist between the surfaces. As $\phi$ is increased, both saddle-node curves form a kink that turns into a "swallowtail" feature that grows in size (Figure 7B and $\mathrm{C}$ ). Inside each swallowtail there are four mixed mode solutions. As $\phi$ is increased further, one end of each of the swallowtails extends toward the other until they eventually meet, joining the two saddle-node surfaces and destroying the swallowtails (Figure 7D and E). The surfaces then exchange ends and split apart so that one surface now 
A

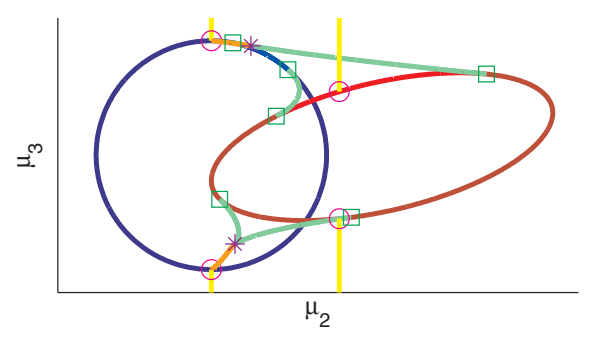

$\mathrm{B}$

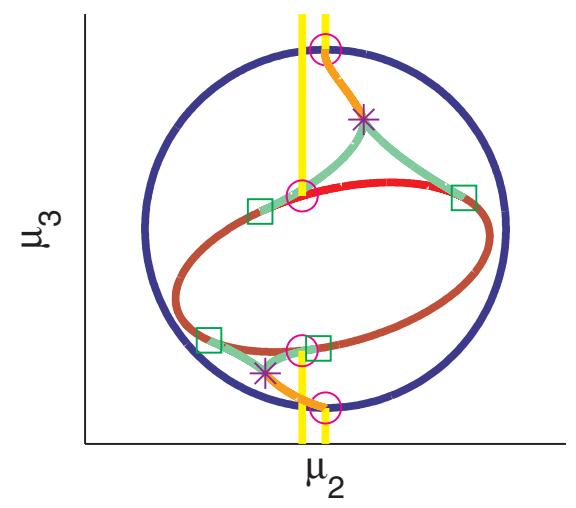

Figure 8. Bifurcation diagrams for systems (53) and (54) on a plane with constant $\mu_{1}$ when the cones are close. A. Intersecting cones showing three branches of the saddle-node surface for mixed mode solutions. Parameter values: $b_{12}=-0.4, b_{21}=-2, b_{31}=-0.5, b_{32}=-0.2, B_{1}=0.1, B_{2}=0.45, \phi=0.6$. The Bogdanov-Takens point on the lower branch is close to but not exactly at the cusp. B. The anti-phase cone completely inside the in-phase cone. Parameters as in A, but $b_{12}=-0.515$. Symbols and colors as in Figure 6 .

starts and ends at the in-phase cone, while the second starts and ends at the anti-phase cone (Figure 7F). The region of four mixed mode solutions is now the area between the intersection of the two surfaces. As $\phi$ is increased further, each cusp moves toward the other portion of the other surface until the cusp eventually meets and rejoins the other surface (Figure 7G). The surfaces reexchange portions of themselves so that each surface now connects to both cones once again, and the swallowtail features have been recreated (Figure 7H). Eventually, as $\phi$ approaches its original value $(\bmod 2 \pi)$, the surfaces again become smooth.

Sometimes the swallowtails in the saddle-node surface appear on the imaginary portion, which, if it were plotted, lies beyond the tangency with the cones. This portion of the saddlenode surface represents bifurcations of equilibria given by (58), but for which one of $r_{1}^{2}$ or $r_{2}^{2}$ is negative. As an appropriate parameter is varied, this swallowtail typically moves past the cone to the real portion of the saddle-node surface, and the effect is that first one fold and then an opposite one appear at the intersection with the cone. This second fold may grow out and meet the fold of the other saddle-node surface before the entire swallowtail has moved into the real portion of the saddle-node surface.

More than two branches of the saddle-node surface are also possible. Figure 8A shows a bifurcation diagram where there are three such surfaces. In this case the cones are intersecting one another, and the number of mixed mode solutions is between zero and three.

6.3.4. Subcritical case. Suppose that the primary Hopf bifurcation for the anti-phase mode is subcritical; that is, $s_{1}=-1$ and $s_{2}=1$ in (48). (Recall that the opposite case when the in-phase mode is subcritical can be transformed to this one, as discussed in section 3.7.) For $s_{2}=1$ the anti-phase normal mode solution is $\left(r_{1}, r_{2}\right)=\left(0, \sqrt{-\mu_{2}}\right)$, exists for $\mu_{2}<0$, and, in contrast to the supercritical case, is always unstable. Besides the absence of stable antiphase normal mode solutions, the main difference between this system and the supercritical case discussed above is that over large regions of the parameter space almost all trajectories 


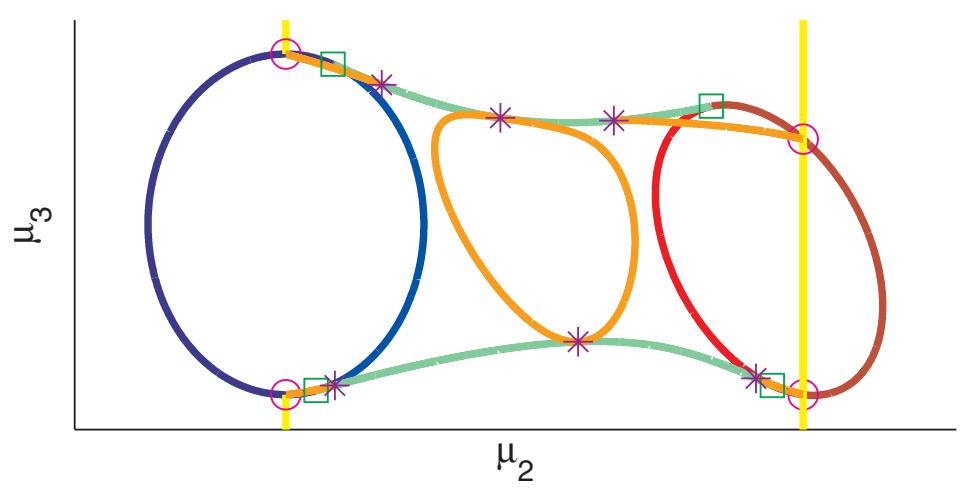

Figure 9. Bifurcation diagram for systems (48) and (50), with $s_{1}=-1$ and $s_{2}=1$, on a plane with constant $\mu_{1}$. A closed curve of Hopf bifurcations (orange) of the 3-dimensional system connect the saddle-node surfaces (green) between the cones. As $\mu_{2}$ is increased across this curve, the mixed mode phase-locked solution loses stability. The stable breather solution which is generated when crossing the in-phase plane (yellow, on the left) above or below the in-phase cone (blue) also loses stability as $\mu_{2}$ is increased. Parameter values: $b_{12}=-1$, $b_{21}=2.5, b_{31}=-0.5, b_{32}=-0.4, B_{1}=0.3, B_{2}=0.4, \phi=2$. Symbols and colors as in Figure 6.

leave a neighborhood of the origin in finite time.

Almost all of the bifurcation analysis done for the supercritical case applies to this case except for stability considerations. In particular, it appears that stable breathers exist with $\mu_{2}$ above but near the in-phase plane, provided the normal mode planes are in inverted orientation, which in this case means

$$
b_{12}<0, \quad b_{21}>0, \quad \text { and } \quad b_{12} b_{21}<-1 .
$$

When (114) holds, the stable mixed mode solutions that arise when $\mu$ crosses the in-phase cone lose stability at a curve of Hopf bifurcations for (48) that span the area enclosed by the saddle-node curves, as shown in Figure 9.

Numerically, it is also evident that the breather solutions lose stability as $\mu$ moves from the in-phase plane toward the anti-phase plane although we have not investigated this bifurcation. In addition, since there are always some trajectories that leave a neighborhood of the origin, the basins of attraction of the stable solutions tend to shrink as one continues moving in parameter space away from where these stable solutions were first born.

7. Conclusions and further work. The main contributions of this work can be summarized as follows. The classical problem of the behavior of two coupled oscillators with Huygens symmetry has been elucidated using ideas of equivariant bifurcation theory. This theory shows that the anti-phase synchronization originally reported by Huygens exists as a consequence of the symmetry of his system, independently of the physical characteristics of the oscillators. The Elphick-Huygens normal form, derived in [22], is shown to be an effective tool for the study of double Hopf bifurcation at 1:1 resonance with Huygens symmetry. The existence of three types of phase-locked periodic solutions is demonstrated: the two normal modes (in-phase and anti-phase) and two (or four) mixed mode periodic solutions that may arise in secondary bifurcations from each of the two normal modes. The escapement paradox is explained. A modification of Krasnoselskii's theorem is used to prove existence of quasiperiodic solutions on a 3 -torus with two frequencies of order one and a third small frequency. 
This 3-torus behaves as a 2-torus "breather" with a slow modulation of its two principal radii. Secondary bifurcations from the normal mode solutions (both in-phase and anti-phase) are studied in detail. These secondary bifurcations give birth either to mixed mode periodic solutions or to quasi-periodic "breather" solutions as above, depending on the values of the parameters relative to an Arnold tongue. This study has focused on the case in which the coefficients $a_{11}$ and $a_{22}$ in the normal form are both negative. In this case both primary Hopf bifurcations are supercritical and stable, and therefore this case is the most relevant for practical problems where only stable behavior is observable.

Some directions of future research under investigation include the study of 1:1 resonance with Huygens symmetry for the cases of the normal form which are not fully explored here, in particular the case when the coefficients $a_{11}$ and $a_{22}$ have opposite signs. This case is more complicated and will require consideration of the fifth order terms of the normal form. More complex dynamics including heteroclinic orbits and strange attractors are under consideration.

Another direction of research is the application of theoretical results obtained here to practical systems with Huygens symmetry, such as pairs of identical neurons $[7,11,18]$ or symmetrically coupled oscillators in physics and engineering; see $[3,4,5,19,23,27,30,31$, $32,33]$. The classic exemplar of symmetrically coupled identical oscillators is Huygens' clocks, as described in 1665 [20]. In 2002, Bennett et al. [4] applied modern engineering methods to the study of Huygens' clocks. They constructed a mechanical apparatus and modeled it mathematically with second order differential equations. However, their study did not exploit the impact of symmetry on the behavior of the system, and they claimed the necessity of including an escapement mechanism in the model. An analysis of Huygens' clocks using the methods and results of this paper is in preparation.

Acknowledgments. The authors thank S. A. Campbell, M. Golubitsky, H. Kunze, and two anonymous referees for helpful suggestions.

\section{REFERENCES}

[1] H. Amann, Ordinary Differential Equations: An Introduction to Nonlinear Analysis, de Gruyter Stud. Math. 13, Walter de Gruyter, Berlin, 1990.

[2] P. Ashwin and J. W. Swift, The Dynamics of $n$ Weakly Coupled Oscillators, J. Nonlinear Sci., 2 (1992), pp. 69-108.

[3] V. N. Belykh and E. V. Pankratova, Chaotic dynamics of two Van der Pol-Duffing oscillators with Huygens coupling, Regul. Chaotic Dyn., 15 (2010), pp. 274-284.

[4] M. Bennett, M. F. Schatz, H. Rockwood, and K. Wiesenfeld, Huygens' clocks, Proc. R. Soc. Lond. A, 458 (2002), pp. 563-579.

[5] I. I. Blekhman, Synchronization in Science and Technology, ASME Press, New York, 1988.

[6] E. Brown, P. Holmes, And J. Moehlis, Globally coupled oscillator networks, in Perspectives and Problems in Nonlinear Science, Springer, New York, 2003, pp. 189-215.

[7] S. A. Campbell and V. G. LeBlanc, Resonant Hopf-Hopf interactions in delay differential equations, J. Dynam. Differential Equations, 10 (1998), pp. 327-346.

[8] P. Chossat and R. Lauterbach, Methods in Equivariant Bifurcations and Dynamical Systems, Adv. Ser. Nonlinear Dynam., World Scientific, Singapore, 2000.

[9] S.-N. Chow, C. Li, And D. Wang, Normal Forms and Bifurcations of Planar Vector Fields, Cambridge University Press, Cambridge, UK, 1994.

[10] C. Elphick, E. Tirapegui, M. E. Brachet, P. Coulet, and G. Iooss, A simple global characterization for normal forms of singular vector fields, Phys. D, 29 (1987), pp. 95-127. 
[11] G. B. Ermentrout and D. H. Terman, Mathematical Foundations of Neuroscience, Springer-Verlag, New York, Berlin, 2010.

[12] M. Golubitsky And W. F. LAngFord, Classifications and unfoldings of degenerate Hopf bifurcations, J. Differential Equations, 41 (1981), pp. 375-415.

[13] M. Golubitsky And I. Stewart, The Symmetry Perspective, Birkhäuser-Verlag, Basel, Switzerland, 2002.

[14] M. Golubitsky, I. Stewart, and D. G. Schaeffer, Singularities and Groups in Bifurcation Theory, Vol. II, Springer-Verlag, New York, 1988.

[15] W. J. F. GovaerTs, Numerical Methods for Bifurcations of Dynamical Equilibria, SIAM, Philadelphia, 2000.

[16] J. Guckenheimer And P. Holmes, Nonlinear Oscillations, Dynamical Systems, and Bifurcations of Vector Fields, Springer-Verlag, New York, Berlin, 1983.

[17] P. Hartman, Ordinary Differential Equations, 2nd ed., Classics Appl. Math. 38, SIAM, Philadelphia, 2002.

[18] F. C. Hoppensteadt and E. M. Izhikevich, Weakly Connected Neural Networks, Springer, New York, 1997.

[19] D. Hurley and M. VAndyck, An observation about the Huygens clock problem, in Two Cultures, K. Williams, ed., Birkhäuser-Verlag, Basel, Switzerland, 2006, pp. 59-70.

[20] C. Huygens, Oeuvres complètes de Christiaan Huygens, vol. 5, Martinus Nijhoff, The Hague, The Netherlands, 1893, pp. 241-262.

[21] G. Iooss And W. F. LAngFord, Conjectures on the routes to turbulence via bifurcation, in Nonlinear Dynamics, R. H. G. Helleman, ed., Annals N. Y. Acad. Sci. 357, New York Academy of Sciences, New York, 1980, pp. 489-505.

[22] P. M. Kitanov, Normal Form Analysis for Bifurcations with Huygens Symmetry, Ph.D. thesis, University of Guelph, Guelph, ON, Canada, 2011.

[23] D. J. Korteweg, Les horloges sympathiques de Huygens, Arch. Neerlandaises, XI (1906), pp. $273-295$.

[24] M. A. KrasnoselskiI, The Operator of Translation along the Trajectories of Differential Equations, Amer. Math. Soc. Transl. Math. Monogr., AMS, Providence, RI, 1968.

[25] Y. Kuznetsov, Elements of Applied Bifurcation Theory, Springer-Verlag, New York, Berlin, 2004.

[26] W. F. LAngford, Periodic and steady-state mode interactions lead to tori, SIAM J. Appl. Math., 37 (1979), pp. 22-48.

[27] A. I. Manevich And L. I. Manevitch, The Mechanics of Nonlinear Systems with Internal Resonances, Imperial College Press, London, 2005.

[28] J. E. Marsden and M. McCracken, The Hopf Bifurcation and Its Applications, Springer-Verlag, New York, 1976.

[29] J. Moenlis And E. Knobloch, Bursts in oscillatory system with broken $\mathbf{D}_{4}$ symmetry, Phys. D, 135 (2000), pp. 263-304.

[30] J. Pantaleone, Synchronization of metronomes, Amer. J. Phys., 70 (2002), pp. 992-1000.

[31] A. Pikovsky, M. Rosenblum, and J. Kurths, Synchronization: A Universal Concept in Nonlinear Science, Cambridge Nonlinear Sci. Ser. 12, Cambridge University Press, Cambridge, UK, 2001.

[32] M. Sentaror, Synchronization of two coupled escapement-driven pendulum clocks, J. Sound and Vibration, 291 (2006), pp. 566-603.

[33] P. H. Steen And S. H. Davis, Quasiperiodic bifurcation in nonlinearly-coupled oscillators near a point of strong resonance, SIAM J. Appl. Math., 42 (1982), pp. 1345-1368.

[34] J. W. Swift, Hopf bifurcation with the symmetry of the square, Nonlinearity, 1 (1988), pp. 333-377.

[35] S. A. VAn Gils, M. KRupa, AND W. F. LANGFORD, Hopf bifurcation with non-semisimple 1:1 resonance, Nonlinearity, 3 (1990), pp. 825-850.

[36] S. A. VAn Gils And M. Silber, On the uniqueness of invariant tori in $\mathbf{D}_{4} \times \mathbf{S}^{1}$ symmetric systems, Nonlinearity, 8 (1995), pp. 615-658.

[37] A. VAnderbauwhede, Hopf bifurcation at nonsemisimple eigenvalues, in Multiparameter Bifurcation Theory, M. Golubitsky and J. Guckenheimer, eds., Contemp. Math. 56, AMS, Providence, RI, 1986, pp. 343-353.

[38] S. Wiggins, Introduction to Applied Nonlinear Dynamical Systems and Chaos, Springer-Verlag, New York, Berlin, 1990. 